\title{
DEVELOPMENT OF A SIMPLE, PORTABLE, COLORIMETRIC ARSENIC SENSOR BASED ON MOLYBDENUM BLUE CHEMISTRY
}

\author{
by \\ Nevetha Yogarajah, BSc \\ (University of Toronto. 2013)
}

A thesis presented to Ryerson University.

\author{
In partial fulfillment of the \\ requirements for the degree of \\ Master of Applied Science \\ in the Program of \\ Environmental Applied Science and Management
}

Toronto, Ontario, Canada, 2016

(C) Nevetha Yogarajah, 2016 


\section{AUTHOR'S DECLARATION FOR ELECTRONIC SUBMISSION OF A THESIS}

I hereby declare that I am the sole author of this thesis. This is a true copy of the thesis, including any required final revisions, as accepted by my examiners.

I authorize Ryerson University to lend this thesis to other institutions or individuals for the purpose of scholarly research.

I further authorize Ryerson University to reproduce this thesis by photocopying or by other means, in total or in part, at the request of other institutions or individuals for the purpose of scholarly research.

I understand that my thesis may be made electronically available to the public. 


\title{
DEVELOPMENT OF A SIMPLE, PORTABLE, COLORIMETRIC ARSENIC SENSOR BASED ON MOLYBDENUM BLUE CHEMISTRY
}

Master of Applied Science, 2016

\author{
Nevetha Yogarajah \\ Environmental Applied Science and Management \\ Ryerson University
}

\begin{abstract}
:
The purpose of this thesis research is to develop a portable sensor for trace arsenic determination, which can be used in developing nations such as Bangladesh. Following an extensive review of the existing literature, I have chosen colorimetric detection as my method of choice for the development of my arsenic sensor.
\end{abstract}

In specific, I have worked to engineer a simplified protocol of the Morita and Kaneko assay, which is based on classical molybdenum-blue chemistry. This assay proceeds by reacting $\mathrm{As}(\mathrm{V})$ in solution with Mo(VI) under oxidizing, acidic conditions; the resultant arsenomolybdate heteropolyacid then reacts with ethyl violet in solution to form stable, coloured ion-aggregates. I have improved the cost, portability, and ease of use of the original assay, via adapting it to a liquid-free paper-based platform. The preliminary assay has an arsenic limit of detection (LOD) of $78 \mu \mathrm{g} / \mathrm{L}$. 
Acknowledgements:

I would like to thank my supervisor, Dr. Scott Tsai, for the opportunity to work on the arsenic detection project. I would also like to thank my colleagues Brian Battaglia, Byeong-Ui Moon, Vaskar Gnyawali, Jasmin Honegger, and Jorvani Cruz.

I addition, I would also like to thank Grand Challenges Canada, the Government of Ontario, and the Yeates School of Graduate Studies for funding this research 
Author's Note:

Much of the supporting research motivating this thesis is based heavily on my first-author critical review paper, "Detection of trace arsenic in drinking water: challenges and opportunities for microfluidics," published in the May 2015 issue of Environmental Science: Water Research and Technology.

Performing a review of the arsenic contamination problem, as well as the existing studies concerning arsenic detection was my first step towards deciding which assay to develop, and also how to go about doing so. During the process of reviewing the literature, I realised that such a review had not yet been published; with guidance from my thesis supervisor Dr. Scott Tsai, I shaped it into a comprehensive review of existing technology and an outline of gaps within the field.

The purpose of the review is to highlight the need for better portable arsenic contamination detection, and describe how microfluidic technology may be developed to address this need. 


\section{TABLE OF CONTENTS}

\section{Front Matter}

Declaration for Submission of a Thesis $\quad$ ii

Abstract $\quad$ iii

Acknowledgements $\quad$ iv

Author's Note $\quad$ V

Introduction 1

Issues of Arsenic Contamination $\quad 1$

Arsenic Testing Efforts

Emerging Arsenic Detection Research

1 Current Status of Arsenic Detection Technology 6

1.1 Currently Used Methods for Arsenic Detection $\quad 6$

$\begin{array}{lll}\text { 1.1.1 Laboratory Methods } & 6\end{array}$

$\begin{array}{lll}\text { 1.1.2 Portable Methods } & 7\end{array}$

1.2 Towards Better Arsenic Detection in Water 13

1.2.1 The Ideal Arsenic Sensor 13

1.2.2 The Merits of Microfluidics 14

1.3 Candidate Methods for Microfluidic Arsenic Detection 15

$\begin{array}{ll}\text { 1.3.1 Colorimetric Methods } & 17\end{array}$

1.3.2 Electrochemical Methods 22

1.3.3 Biological Methods 24

1.3.4 Electrophoretic Methods 28

1.3.5 Surface Sensing Methods 30

1.3.6 Spectroscopic Methods $\quad 32$

1.4 Micro Total Analytical Systems ( $\mu$ TASs) for Arsenic Detection 36

1.4.1 Specific Concerns for Arsenic Analysis 36

1.4.2 General Issues of Portability 38

$\begin{array}{lll}1.5 & \text { Summary } & 40\end{array}$

2 Development of a Portable Platform for Arsenic Detection 41

$\begin{array}{lll}2.1 & \text { Introduction } & 41\end{array}$

2.2 $\mu$ PAD Adaptation of the Morita and Kaneko Assay 44 
2.2.1 Characterisation of the Bulk Assay 44

2.2.2 Wicking-based $\mu$ PADs 45

2.2.3 Immersion-based $\mu$ PADs $\quad 51$

2.3 Elimination of Liquid Reagents from the $\mu$ PAD Sensor 55

2.3.1 Determination of Reagent Incompatibilities 55

2.3.2 Liquid-free Reagent Delivery 56

2.3.3 Reagent Introduction via DEX Pellets 58

2.4 Summary $\quad 63$

Conclusions and Future Directions $\quad 65$

$\begin{array}{ll}\text { Appendices } & 67\end{array}$

$\begin{array}{ll}\text { General Methodological Features } & 67\end{array}$

$\begin{array}{ll}\text { Solution Preparation } & 67\end{array}$

$\begin{array}{ll}\text { Paper Device Fabrication } & 68\end{array}$

$\begin{array}{ll}\text { Image Acquisition and Analysis } & 69\end{array}$

$\begin{array}{ll}\text { References } & 70\end{array}$ 


\section{INTRODUCTION}

The fundamental purpose of this research is to evaluate the utility of microfluidics with regards to portable trace arsenic detection, and to explore the development of a simple portable arsenic sensor that can potentially be use for routine monitoring in arsenic affected regions. This work is motivated by the need for a better portable arsenic sensor for routine arsenic monitoring in regions such as Bangladesh, and the hope that the development of better technology may lead the lowering of their current arsenic maximum contamination limit of $50 \mu \mathrm{g} / \mathrm{L}$ down to the World Health Organisation suggested standard of $10 \mu \mathrm{g} / \mathrm{L}$. My initial step towards this research was to carry out an extensive review of the literature to determine the current state of existing arsenic detection technology. Having gained an appreciation of the abilities and limitations of current arsenic detection technology with respect to routine monitoring, I then proceeded to engineer my own portable colorimetric sensor for arsenic determination, based on molybdenum blue chemistry. If successfully refined, the portable assay that I have presented for arsenic detection can be commercialised for routine arsenic monitoring in affected regions. These two distinct stages of my research are described below, in Chapters 1 and 2 respectively.

\section{ISSUES OF ARSENIC CONTAMINATION}

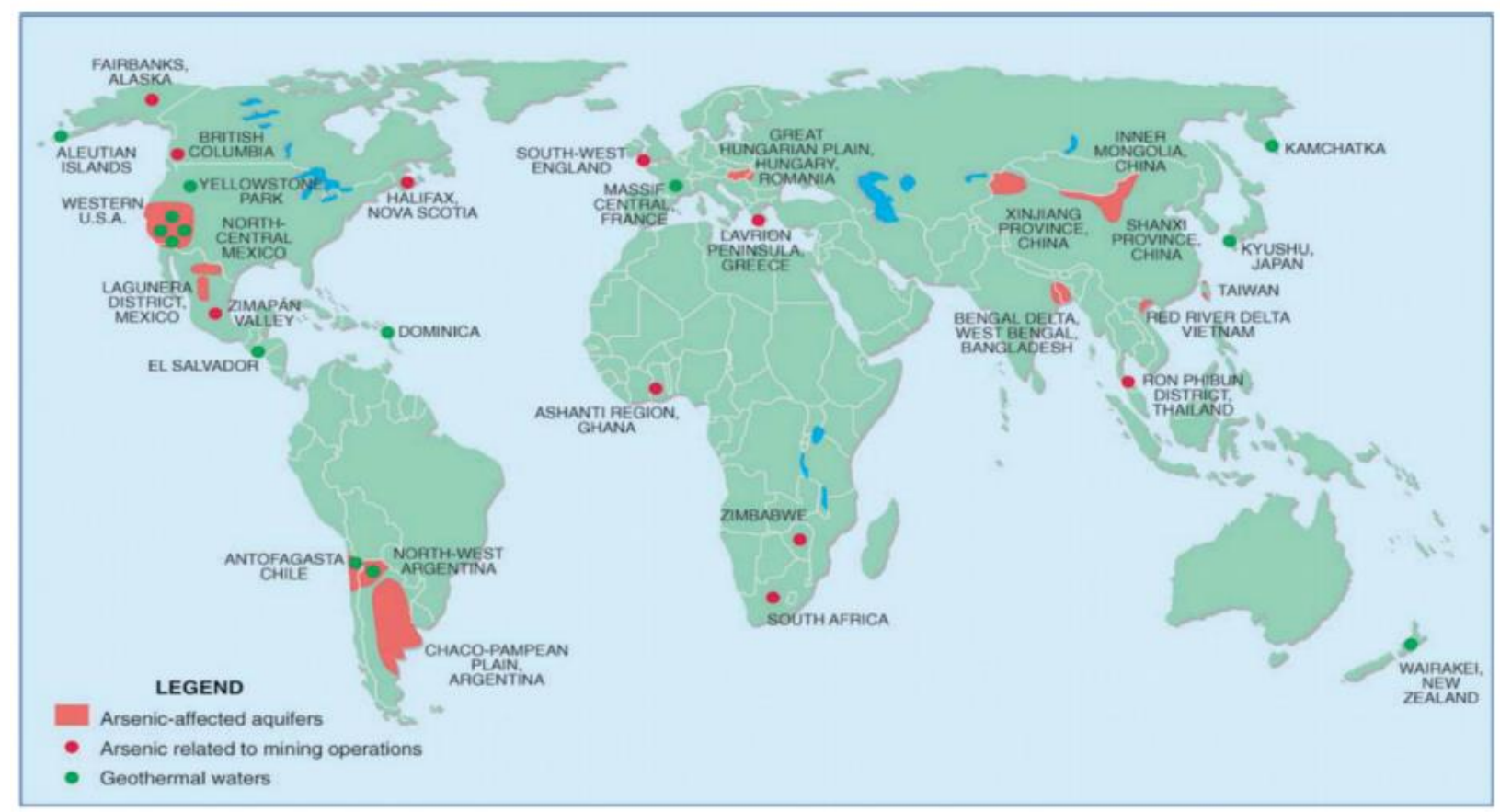

Figure 1: Map depicting global arsenic occurrence; as indicated in the legend, arsenic-affected aquifers are highlighted in red, areas with arsenic related to mining are indicated by red dots, and geothermal waters are indicated by green dots (Smedley \& Kinniburgh 2002). 
Arsenic contamination affects regions in all corners of the world. Among others, contaminated areas include Argentina, Bangladesh, China, India, Mexico, Myanmar, Nepal, Pakistan, Vietnam, and parts of the USA (Figure 1) (Petrusevski \& Sharma 2007; World Bank Water and Sanitation Program 2005). Specifically, contamination of aquifers in Bangladesh is deemed most serious; consequently, a great deal of research efforts and field studies concerning arsenic contamination and mitigation have been focused on this region.

An estimated 35-77 million Bangladeshi people, or 28-62\% of the nation's population, are at risk of exposure to arsenic contaminated water (Smith et al. 2000). The arsenic epidemic in Bangladesh is described as "the largest mass poisoning of a population in history" (Smith et al. 2000; Kinniburgh \& Smedley 2001). A prospective cohort study following almost 12,000 Bangladeshis over the course of 10 years, from 2000-2009, reported that nearly $20 \%$ of all deaths in the nation are due to arsenic (Argos et al. 2010). In some parts of Bangladesh, arsenic levels in water are up to $2,500 \mu \mathrm{g} / \mathrm{L}$; the source of this contamination is attributed to a naturally high percent composition of the element within ground sediments (WHO 2001; Petrusevski \& Sharma 2007; Cicero 2009). As arsenic leaching is a continual process, it is expected that well testing must also be carried out in an ongoing manner.

Arsenic poisoning incurred from chronic exposure to high levels of arsenic is referred to as arsenicosis. Symptoms of this condition include skin lesions and hard patches on the palms of hands and soles of feet; skin and internal organ cancers; diseases of blood vessels in the legs; and also diabetes, high blood pressure, and reproductive disorders (WHO 2001; Yang et al. 2008; Chen et al. 1986; Wu et al. 1989; Ferreccio et al. 2000; Hopenhayn-Rich et al. 1998; Tsuda et al. 1995; Morales et al. 2000; Baastrup et al. 2008; Argos et al. 2010). Arsenicosis severity is highly dependent on the dose and number of years of exposure to arsenic (Ferreccio et al. 2000; Tsuda et al. 1995; Wu et al. 1989; Morales et al. 2000; Argos et al. 2010; Sohel et al. 2009; Yang et al. 2004; Lamm et al. 2004; Tseng 2002; Villanueva \& Kogevinas 1999). The current World Health Organisation (WHO) maximum contamination limit (MCL) for arsenic in drinking water, defined in 1993, is $10 \mu \mathrm{g} / \mathrm{L}$ (WHO 2011). This limit is based on the projected health effects of lifetime exposure to arsenic (Smith et al. 2000).

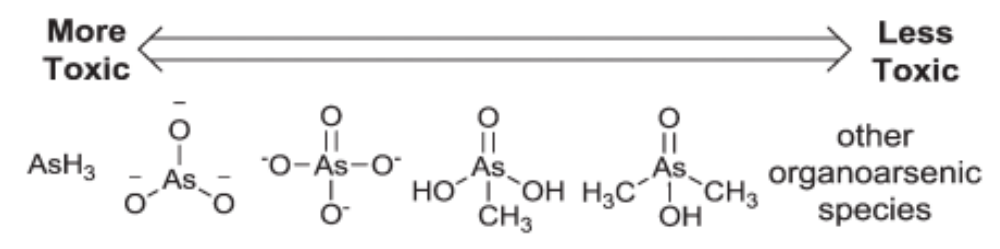

Figure 2: Arsenic species that may be generated or encountered in water analyses; in order of decreasing toxicity (from left to right): arsine gas, arsenite oxoanion, arsenate oxoanion, monomethylarsonic acid (MMA), dimethylarsinic acid (DMA), and other organoarsenic species. 
In solution, arsenic is readily converted from one species to another via chemical and biological redox pathways. Since arsenic speciation determines both its bioavailability and its potency as a toxin, there is much interest in the literature for speciation studies of arsenic. Generally speaking, these studies are difficult and expensive to carry out, and so they are not 'first response measures'. As a result, arsenic speciation will not be extensively focused upon in this work. For the current status of the field, I direct you to a recent review by Chen et al (Chen et al. 2014). I find the As(III) and As(V) arsenic species to be of greatest interest for primary analyte detection because they are the most common forms of arsenic in water, and pose high toxicity to humans (Figure 2) (Samanta et al. 1999).

\section{ArSenic Testing EfForts}

An estimated 6-11 million tube-wells in Bangladesh need to be monitored for arsenic contamination (Jakariya et al. 2007). The current arsenic standard in Bangladesh, for drinking water, is $50 \mu \mathrm{g} / \mathrm{L}$ (Flanagan et al. 2012; Petrusevski \& Sharma 2007). Given the magnitude of contamination, and the socioeconomic status of the nation, it is not feasible for Bangladesh to adhere to the provisional WHO standard of $10 \mu \mathrm{g} / \mathrm{L}$. Furthermore, Bangladesh and India have adopted a system of painting arsenic-tested wells either green or red, to indicate 'safe' and 'unsafe' water sources respectively. Since, for arsenic, the primary mode of entry into the body is through ingestion and inhalation rather than absorption, 'unsafe' wells can still be used for non-consumption purposes such as washing clothes (WHO 2001).

Established laboratory techniques such as hydride generation atomic absorption spectroscopy (HG AAS), inductively coupled plasma mass spectrometry (ICP MS), and atomic fluorescence spectroscopy (AFS) can easily measure arsenic levels below $10 \mu \mathrm{g} / \mathrm{L}$ (Behari \& Prakash 2006; Klaue \& Blum 1999; Wahed et al. 2006; United States Environmental Protection Agency Office of Water 1999; Roje et al. 2007). While highly sensitive, these techniques are bulky, expensive, and require highly trained personnel for their maintenance and operation. As an additional drawback, laboratory detection also involves labelling and shipping of samples to a central facility. Developing nations do not have the infrastructure to perform these procedures reliably and with high throughput.

In light of these issues, the WHO, the United Nations International Children's Emergency Fund (UNICEF), and other organisations use portable test kits for arsenic analysis. Over one million tube-wells in Bangladesh and India have been tested and labelled using portable arsenic test kits (Rahman et al. 2002). Most kits on the market are based on the Gutzeit reaction, known since the 1900s (Sanger \& Black 1907). Here, colorimetric detection is based on the reaction of arsine gas with mercuric bromide embedded on a paper strip; both of these reactants are toxic and hazardous to the user. Independent studies have shown that these kits have a high rate of false negative and false positive results, and are unreliable for the determination of arsenic levels below $70 \mu \mathrm{g} / \mathrm{L}$ (Arora et al. 2009; Jakariya et al. 2007; Kinniburgh \& 
Kosmus 2002; Rahman et al. 2002). As a result, many people continue to drink contaminated waters from 'clean' sources. Furthermore, many people in these regions are still waiting for the drinkability of their water sources to be tested. The Bangladesh Arsenic Mitigation and Water Supply Program (BAMWSP), which conducted arsenic blanket testing between the years 2000-2005, is the most recent national campaign to test tube-wells for arsenic contamination (George et al. 2012).

It is expected that 1 out of 100 people who consume water in excess of $50 \mu \mathrm{g} / \mathrm{L}$ of arsenic, will die specifically due to an arsenic-related cancer (WHO 2001). The development of an arsenic sensor, with the detection power of a laboratory method and cost and convenience of a portable kit, is vital to control the number of people exposed to this pollutant. Many groups, including my own, are working on the advancement of alternate analytical methods for arsenic detection; however, a comprehensive solution is yet to be developed. To be successfully used in developing nations, I propose that a new arsenic sensor should be: sensitive and selective for arsenic, quick and reliable, portable and robust, health and environmental risk free, affordable and easy to use for local technicians.

\section{EMERging ArSENic Detection RESEARCH}

Microfluidics is an emerging field which focuses on the development of miniaturized, integrated lab-on-a-chip (LOC) devices. In the past 25 years, there has been a surge of interest in the field as researchers miniaturize traditional macro-scale processes to micro-dimensions, and explore new aspects of science previously unseen from a macro-scale vantage point. This technology has been used by studies in a range of applications, from clinical medicine and microbiology, to electronics and the oil industry (Hettiarachchi et al. 2007; Gómez-sjöberg et al. 2005; Bindiganavale et al. 2012; Fadaei et al. 2011). In comparison to their macro-scale counterparts, microfluidic processes have the following advantages: faster reaction times and better process-control; reduced waste generation and reagent consumption; system compactness and parallelization; and reduced cost and disposability.

Several groups are now exploring the use of microfluidics for arsenic and other heavy metal detection. The inherent portability of microfluidics, coupled with the successful employment of LOC devices in other fields, readily lends this technology for the development of practical arsenic sensors. Here, I review the potential use of microfluidics with new and emerging analytical techniques for portable arsenic detection. While there are many reviews of existing arsenic detection techniques, to the best of my knowledge, this is the first review to present an analysis of microfluidic technology in arsenic detection (Melamed 2005; Hung et al. 2004; Francesconi \& Kuehnelt 2004; Arora et al. 2009; Luong et al. 2007).

The present thesis is divided into three broad sections, 1) a literature review outlining the current status of arsenic detection technology, 2) a description of the progress I have made towards the development of a portable platform for arsenic detection, and 3) a discussion about the potential future of the field. In 
the literature review, I first describe existing laboratory and field methods for arsenic detection; and the challenges they face. I then outline what I postulate are characteristics of an ideal, routine arsenic sensor, and also give the advantages of using a microfluidic platform to effect these qualities. Using these criteria as a guideline, I then describe alternate emerging techniques for arsenic detection, and the role, or potential role, of microfluidics in their development. From this analysis, I have chosen to focus my experimental research on the adaptation of an existing colorimetric assay for arsenic detection into a portable platform. In the experimental section I describe the steps I have taken to miniaturise the molybdenum-blue based system for arsenic detection developed by Morita and Kaneko (Morita \& Kaneko 2006a). In closing, I paint a description of advancements in microfluidic technology towards the feasibility of a micro total analytical system ( $\mu$ TAS) for arsenic detection. 


\section{Current Status of Arsenic Detection TeChnology}

\subsection{Currently USED Methods For ArSenic Detection}

\subsubsection{Laboratory Methods}

As cited by Bose et al., laboratory techniques that can be used for arsenic analysis include: atomic spectroscopy, mass spectrometry, neutron activated analysis, electrophoresis, chromatography, potentiometry, and voltammetry (Bose et al. 2011). Most accepted laboratory methods have a limit of detection (LOD) for arsenic on the order of $1 \mu \mathrm{g} / \mathrm{L}$ or 1 part per billion (ppb) (United States Environmental Protection Agency Office of Water 1999). All of these listed techniques can accurately perform high throughput sample analyses with good reproducibility. However, this remarkable detection power is accompanied by an upfront cost upwards of $\$ 30,000$ USD; not to mention the cost of maintenance, consumables, and highly trained technicians (United States Environmental Protection Agency Office of Water 1999).

Table 1: General comparison of the performance attributes of atomic absorption spectroscopy (AAS), inductively-coupled plasma mass spectrometry (ICP MS) and atomic fluorescence spectroscopy (AFS) for trace arsenic detection in water samples. The limit of detection, reproducibility (\% relative standard deviation), required sample size, analysis time, basic instrument cost, necessary skill for instrument operation, and type of data generated are tabulated for each method.

\begin{tabular}{|c|c|c|c|}
\hline & AAS & ICP MS & AFS \\
\hline Limit of Detection $(\mu \mathrm{g} / \mathrm{L})$ & $0.0009-1$ & $0.0003-1$ & $0.0003-10$ \\
\hline Reproducibility (\% RSD) & $<10$ & $<10$ & $<10$ \\
\hline Sample Size $(\mu \mathbf{L})$ & $>1,000$ & $2-200$ & $20-200$ \\
\hline Time Required & $\sim 30$ minutes & $\sim 30$ seconds & $\sim 10$ minutes \\
\hline Cost (USD) & $\sim \$ 60,000$ & $\sim \$ 200,000$ & $\sim \$ 60,000$ \\
\hline Skill Requirement & $\begin{array}{l}\text { Easy to use for a trained } \\
\text { technician }\end{array}$ & $\begin{array}{l}\text { Difficult initial method } \\
\text { development even for a } \\
\text { trained technician }\end{array}$ & $\begin{array}{l}\text { Easy to use for a } \\
\text { trained technician }\end{array}$ \\
\hline Data Type & $\begin{array}{l}\text { Ground state and } \\
\text { resonance transition } \\
\text { optical spectra }\end{array}$ & $\begin{array}{l}\text { Mass-charge peak } \\
\text { spectra, simpler than } \\
\text { optical spectra }\end{array}$ & $\begin{array}{l}\text { Ground state } \\
\text { transition optical } \\
\text { spectra }\end{array}$ \\
\hline References & $\begin{array}{c}\text { (Wahed et al. 2006; } \\
\text { Behari \& Prakash 2006; } \\
\text { Rahman et al. 2002; } \\
\text { Deng et al. 2013; } \\
\text { Jakariya et al. 2007; } \\
\text { United States } \\
\text { Environmental } \\
\text { Protection Agency } \\
\text { Office of Water 1999; } \\
\text { Skoog et al. 2006) }\end{array}$ & $\begin{array}{l}\text { (Klaue \& Blum 1999; } \\
\text { Francesconi \& } \\
\text { Kuehnelt 2004; Roje et } \\
\text { al. 2007; Gómez-Ariza } \\
\text { et al. 2000; Lindberg et } \\
\text { al. 2007; United States } \\
\text { Environmental } \\
\text { Protection Agency } \\
\text { Office of Water 1999; } \\
\text { Skoog et al. 2006) }\end{array}$ & $\begin{array}{l}\text { (Deng et al. 2013; } \\
\text { Gómez-Ariza et al. } \\
\text { 2000; Lindberg et al. } \\
\text { 2007; Steinmaus et al. } \\
\text { 2006; United States } \\
\text { Environmental } \\
\text { Protection Agency } \\
\text { Office of Water 1999; } \\
\text { Skoog et al. 2006) }\end{array}$ \\
\hline
\end{tabular}




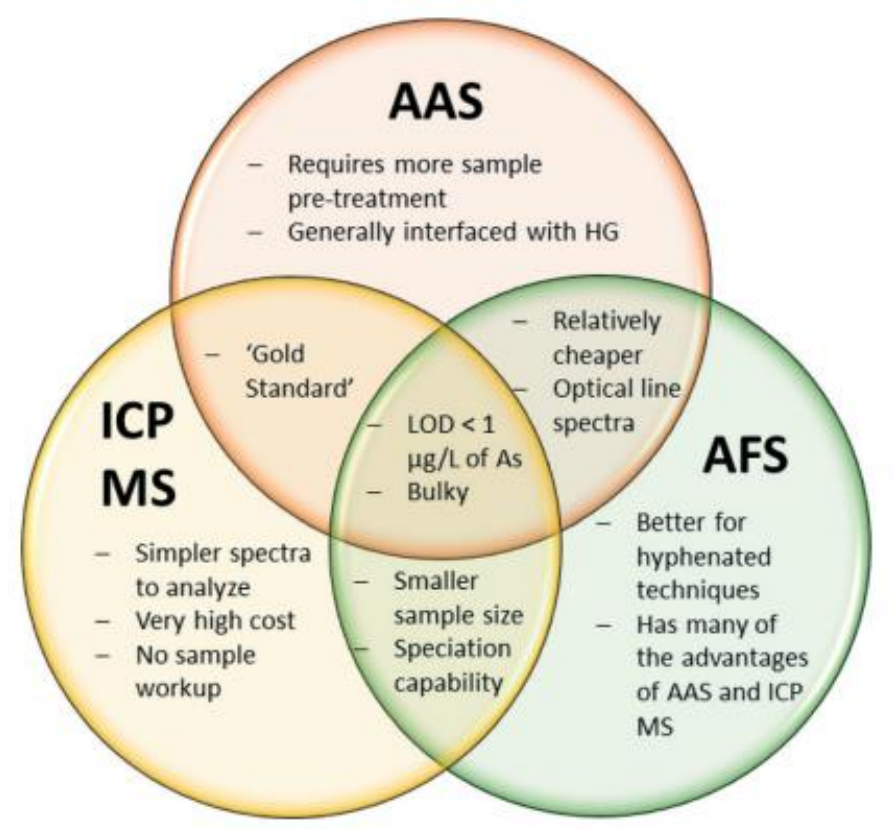

Figure 3: Venn diagram comparing the utility of atomic absorption spectroscopy (AAS), inductivelycoupled plasma mass spectrometry (ICP MS), and atomic fluorescence spectroscopy (AFS). All three of these analytical techniques can detect arsenic with very high sensitivity and selectivity; and HG AAS and ICP MS are analytical 'gold standards' for arsenic detection. AAS is a relatively cheaper technique than ICP MS; but involves difficult sample preparation procedures, requires larger sample volumes, and produces more complex spectra. Conversely, ICP MS is a much more expensive technique; but involves very little sample preparation, can be interfaced with upstream speciation, and produces easily read spectra. AFS detection enjoys many of the individual advantages of both AAS and ICP MS; namely relatively lower cost, and speciation ability.

Specifically, atomic absorption spectroscopy (AAS), mass spectrometry (MS), and atomic fluorescence spectroscopy (AFS) are the favoured techniques of choice used in the literature for calibration and validation of alternate arsenic detection methods. Table 1 lists some general performance attributes such as limit of detection, reproducibility, required sample volume and time per analysis, as well as the costs and expertise needed to run each of these three methods. Although they are powerful analytical tools, the high cost and maintenance associated with laboratory methods renders them unsuitable for routine arsenic monitoring in developing regions (Figure 3).

\subsubsection{Portable Methods}

In the 1990s, an epidemic of arsenic poisoning emerged in Bangladesh as a result of widespread use of arsenic contaminated tube-wells. Tube-wells had been used in Bangladesh since the 1940s, but their widespread use did not begin until the 1970s. Over one million additional wells were added to the region 
to address the emergence of diarrheal disease caused by bacterial colonization of surface waters (Smith et al. 2000).

It was later discovered that many of these tube-wells contained arsenic contaminated water. Organisations such as the WHO, UNICEF and the World Bank unanimously decided to combat the problem. Millions of US dollars have since been invested in the development of cheap and portable field kits for arsenic detection. Many of the introduced arsenic tests were designed to comply with the Bangladeshi arsenic MCL of $50 \mu \mathrm{g} / \mathrm{L}$. Table 2 organizes different arsenic field test kits and compares selected performance attributes pertaining to their suitability for use as a routine test; compared attributes include limit of detection, reliability, cost and time per analysis, as well as skill level required of the technician.

Table 2: General comparison of the performance attributes of various portable arsenic test kits based on the Gutzeit reaction. The theoretical and practical limits of detection (LODs), reliability, cost and time requirement per single analysis, necessary skill requirement, and type of data generated are tabulated for each method. Blank fields are representative of a lack of data in the literature.

\begin{tabular}{|c|c|c|c|c|c|c|c|c|}
\hline & \multicolumn{8}{|c|}{ Portable Arsenic Test Kits Based on the Gutzeit Reaction } \\
\hline & NIPSOM & Merck & GPL & $\begin{array}{l}\text { AAIH } \\
\text { \&PH }\end{array}$ & AAN & Quick As & Hach EZ & Arsenator \\
\hline $\begin{array}{l}\text { Theoretical } \\
\text { LOD }(\mu \mathrm{g} / \mathrm{L})\end{array}$ & 10 & 10 & 10 & 50 & 10 & 5 & 10 & $0.5-2$ \\
\hline $\begin{array}{l}\text { Practical LOD } \\
(\mu \mathrm{g} / \mathrm{L})\end{array}$ & $>20$ & $>50$ & & $>50$ & $>20$ & & & \\
\hline Reliability & $\begin{array}{l}\text { Unreliable } \\
<70 \mu \mathrm{g} / \mathrm{L} \text {. }\end{array}$ & $\begin{array}{c}\text { Unreliable } \\
<70 \mu \mathrm{g} / \mathrm{L} \text {. } \\
\text { Very poor } \\
\text { correlation } \\
\text { with labs. }\end{array}$ & $\begin{array}{l}\text { Unreliable } \\
<70 \mu \mathrm{g} / \mathrm{L} \text {. }\end{array}$ & $\begin{array}{l}\text { Unreliable } \\
<70 \mu \mathrm{g} / \mathrm{L} \text {. }\end{array}$ & $\begin{array}{l}\text { Unreliable } \\
<70 \mu \mathrm{g} / \mathrm{L} \text {. }\end{array}$ & $\begin{array}{c}\text { Can } \\
\text { identify } \\
\text { samples } \\
\text { with over } \\
15 \mu \mathrm{g} / \mathrm{L} \text { of } \\
\text { arsenic as } \\
\text { being over } \\
\text { WHO } \\
\text { limit. }\end{array}$ & $\begin{array}{c}\text { Can identify } \\
\text { samples } \\
\text { with over } \\
15 \mu \mathrm{g} / \mathrm{L} \text { of } \\
\text { arsenic as } \\
\text { being over } \\
\text { WHO limit. }\end{array}$ & $\begin{array}{l}\text { Found to be } \\
\text { correct } 85 \% \\
\text { of the time. } \\
\text { More } \\
\text { reliable at } \\
\text { lower } \\
\text { concentrat- } \\
\text { ions. }\end{array}$ \\
\hline $\begin{array}{l}\text { Cost per sample } \\
\text { (USD) }\end{array}$ & $\begin{array}{l}\$ 0.40 \\
\$ 2.00^{*}\end{array}$ & $\begin{array}{c}\$ 0.50- \\
1.00, \\
\$ 2.00^{*}\end{array}$ & $\begin{array}{l}\$ 0.40 \\
\$ 2.00^{*}\end{array}$ & $\begin{array}{l}\$ 0.40 \\
\$ 2.00^{*}\end{array}$ & $\begin{array}{l}\$ 0.40 \\
\$ 2.00^{*}\end{array}$ & $\begin{array}{l}\$ 1.00- \\
2.00\end{array}$ & $\begin{array}{c}<\$ 1.00- \\
2.00\end{array}$ & $\begin{array}{l}\$ 1.00 \\
\$ 9.00 * *\end{array}$ \\
\hline $\begin{array}{l}\text { Time per sample } \\
\text { (min) }\end{array}$ & 5 & 30 & 20 & & 30 & & $20-40$ & 20 \\
\hline $\begin{array}{l}\text { Skills required } \\
\text { by technician }\end{array}$ & $\begin{array}{c}\text { Colour } \\
\text { sensitivity } \\
\text { to yellow; } \\
\text { working } \\
\text { quickly. }\end{array}$ & $\begin{array}{c}\text { Colour } \\
\text { sensitivity } \\
\text { to yellow; } \\
\text { working } \\
\text { quickly. }\end{array}$ & $\begin{array}{c}\text { Colour } \\
\text { sensitivity } \\
\text { to yellow; } \\
\text { working } \\
\text { quickly. }\end{array}$ & $\begin{array}{c}\text { Colour } \\
\text { sensitivity } \\
\text { to yellow; } \\
\text { working } \\
\text { quickly. }\end{array}$ & $\begin{array}{c}\text { Colour } \\
\text { sensitivity } \\
\text { to yellow; } \\
\text { working } \\
\text { quickly. }\end{array}$ & $\begin{array}{c}\text { Colour } \\
\text { sensitivity } \\
\text { to yellow; } \\
\text { working } \\
\text { quickly. }\end{array}$ & $\begin{array}{c}\text { Colour } \\
\text { sensitivity } \\
\text { to yellow; } \\
\text { working } \\
\text { quickly. }\end{array}$ & $\begin{array}{l}\text { Ability to } \\
\text { make } \\
\text { accurate } \\
\text { dilutions. }\end{array}$ \\
\hline Data type & $\begin{array}{l}\text { Colour } \\
\text { change } \\
\text { (range) }\end{array}$ & $\begin{array}{l}\text { Colour } \\
\text { change } \\
\text { (range) }\end{array}$ & $\begin{array}{l}\text { Colour } \\
\text { change } \\
\text { (range) }\end{array}$ & $\begin{array}{l}\text { Colour } \\
\text { change } \\
\text { (binary) }\end{array}$ & $\begin{array}{l}\text { Colour } \\
\text { change } \\
\text { (range) }\end{array}$ & $\begin{array}{l}\text { Colour } \\
\text { change } \\
\text { (range) }\end{array}$ & $\begin{array}{l}\text { Colour } \\
\text { change } \\
\text { (range) }\end{array}$ & $\begin{array}{l}\text { Digital } \\
\text { readout }\end{array}$ \\
\hline
\end{tabular}




\begin{tabular}{|c|c|c|c|c|c|c|c|c|}
\hline & \multicolumn{8}{|c|}{ Portable Arsenic Test Kits Based on the Gutzeit Reaction } \\
\hline & NIPSOM & Merck & GPL & $\begin{array}{l}\text { AAIH } \\
\text { \&PH }\end{array}$ & $\mathbf{A A N}$ & Quick As & Hach EZ & Arsenator \\
\hline References & $\begin{array}{l}\text { (Rahman } \\
\text { et al. } \\
\text { 2002; } \\
\text { Pande et } \\
\text { al. 2001) }\end{array}$ & $\begin{array}{c}\text { (Rahman } \\
\text { et al. } \\
\text { 2002; } \\
\text { Pande et } \\
\text { al. 2001; } \\
\text { Arora et } \\
\text { al. 2009; } \\
\text { Kinniburg } \\
\text { h \& } \\
\text { Kosmus } \\
\text { 2002) }\end{array}$ & $\begin{array}{c}\text { (Rahman } \\
\text { et al. } \\
\text { 2002) }\end{array}$ & $\begin{array}{l}\text { (Rahman } \\
\text { et al. } \\
\text { 2002; } \\
\text { Pande et } \\
\text { al. 2001) }\end{array}$ & $\begin{array}{l}\text { (Rahman } \\
\text { et al. } \\
\text { 2002; } \\
\text { Pande et } \\
\text { al. 2001; } \\
\text { Kinniburg } \\
\text { h \& } \\
\text { Kosmus } \\
\text { 2002) }\end{array}$ & $\begin{array}{c}\text { (Steinmau } \\
\text { s et al. } \\
2006)\end{array}$ & $\begin{array}{l}\text { (Steinmaus } \\
\text { et al. 2006; } \\
\text { Kinniburgh } \\
\text { \& Kosmus } \\
2002 \text { ) }\end{array}$ & $\begin{array}{l}\text { (Kinniburgh } \\
\& \text { Kosmus } \\
2002 ; \\
\text { Safarzadeh- } \\
\text { Amiri et al. } \\
\text { 2011; } \\
\text { Sankararam } \\
\text { akrishnan et } \\
\text { al. 2008) }\end{array}$ \\
\hline
\end{tabular}

To date, over one million tube-wells have been tested using these arsenic field kits. Upon testing, wells with arsenic levels below $50 \mu \mathrm{g} / \mathrm{L}$ were painted green and the water was deemed 'safe' for consumption. Wells with arsenic levels above this limit were painted red and deemed 'unsafe'; the majority of the wells tested were labelled as unsafe (Rahman et al. 2002).

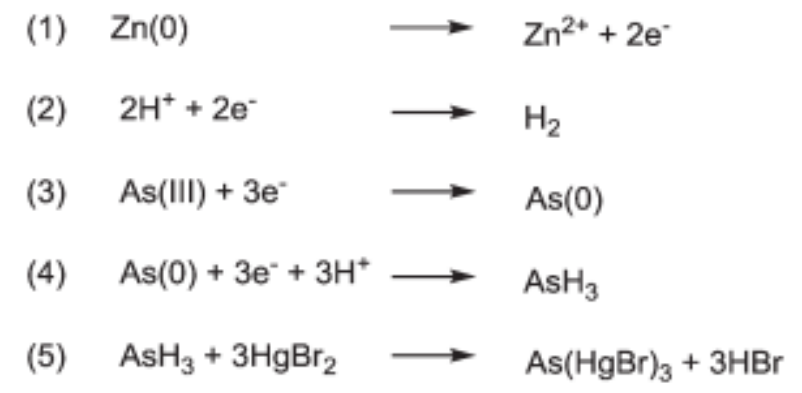

Figure 4: General scheme of the modified Gutzeit reaction used by arsenic test strips; (1-4) generation of arsine gas, (5) generation of coloured $\mathrm{As}(\mathrm{HgBr})_{3}$ (Brindle 2007).

All test kits employed in these original initiatives are based on a one-hundred-year-old method known as the Gutzeit reaction (Sanger \& Black 1907). The kits proceed by first reducing all arsenic in solution to the highly toxic arsine gas, and then reacting the evolved gas with mercuric bromide embedded paper to induce a colour change Figure 4. Upon reaction, depending on the number of $-\mathrm{AsH}_{2}$ groups that are bound to the mercury, the paper strip becomes a yellowish colour of varied intensity.

\subsubsection{Field Kit Evaluations}

Due to the overwhelming number of tube-wells that remain to be tested for arsenic contamination, it is almost certain that most wells will be tested only once. Therefore, the reliability of that single 
determination is of utmost importance. During the late 2000s, several independent groups set out to evaluate the merit of the different available test kits. The results of these studies are quite alarming.

Reported findings indicate that the original test kits yield high frequencies of well mislabelling as either false positive or false negative; that several of the original field kits are unreliable for the detection of arsenic concentrations below $70 \mu \mathrm{g} / \mathrm{L}$; and that visual perception of "yellowness" varies greatly from person to person (Rahman et al. 2002). It has even been suggested that well samples around the $50 \mu \mathrm{g} / \mathrm{L}$ threshold should be routinely re-analysed for verification of the determination (Jakariya et al. 2007). Others have also pointed out that during analysis, nearly $50 \%$ of the evolved arsine gas escapes to the environment before detection (Arora et al. 2009). Arsine gas is more toxic than arsenic in solution; and technician exposure to this compound is of great concern.

Studies of newer generation Gutzeit method-based field test kits have generally been none the more favourable. It has been concluded that none can be used to determine arsenic levels of $10 \mu \mathrm{g} / \mathrm{L}$; and that the lowest detectable arsenic concentration is $20 \mu \mathrm{g} / \mathrm{L}$ (Pande et al. 2001; Deshpande \& Pande 2005). In some cases, improved accuracy has been cited with increased reaction times (Mukherjee et al. 2005). In other cases, it has been further sought to improve the accuracy of the methods via even longer reaction times and imaging of the test strips using a flatbed scanner (Kearns \& Tyson 2012). A study from Nebraska, USA looking at the Quick Arsenic and Hach EZ kits has concluded favourably with regards to their routine use (Steinmaus et al. 2006). Evaluating against the US Environmental Protection Agency (US EPA) standard of $10 \mu \mathrm{g} / \mathrm{L}$ of arsenic in solution, the authors have found that, largely, water samples containing $15 \mu \mathrm{g} / \mathrm{L}$ or more of arsenic were correctly identified as exceeding the MCL. Consequently, the authors suggest that these particular field kits are reliable for use in arsenic detection and remediation initiatives. I note that this is a minority point of view, as most evaluations of Gutzeit method-based field test kits conclude that the kits are unreliable for testing near the $10 \mu \mathrm{g} / \mathrm{L}$ arsenic toxicity concentration threshold.

\subsubsection{Issues with Existing Field Kits}

Below I highlight some problems associated with the Gutzeit method of arsenic detection, as presented by Kinniburgh et al (Kinniburgh \& Kosmus 2002).

1) The human eye is not very sensitive to the colour yellow.

There is a large person to person variability in the detection of the degree of yellow-ness developed; this subsequently leads to discrepancies in sample classifications.

2) The evolved yellow colour fades with time.

Determinations must be made quickly; and, in order to be comparable to one another, must all be taken after a uniform time lapse. 
3) Mercuric halide test strips are sensitive to hydrogen sulphide $\left(\mathrm{H}_{2} \mathrm{~S}\right)$ interference.

$\mathrm{H}_{2} \mathrm{~S}$ produces a black stain when bound to mercury, and thereby renders the test strip useless. Some newer methods, address this by including additional reagents to remove the sulphur interference (Das et al. 2014).

4) There are difficulties in working with zinc.

Zinc serves as a reducing agent in the reaction. Zinc ores naturally contain high levels of arsenic, so this may introduce error in the determination. In some cases, this issue is addressed via reduction by sodium borohydride instead (Das et al. 2014).

The usefulness of a test derives from the information it produces (Kinniburgh \& Kosmus 2002). So, if arsenic field kits cannot reliably classify a water source as either 'safe' or 'unsafe', then they do not serve the purpose for which they are were designed.

\subsubsection{Digital Interpretation of Field Kits}

An approach to overcome some of these problems is through digital detection of the colour change. Electronic kits such as the Wagtech Arsenator drastically increase the precision of field test measurements. However, while one study has found Arsenator determinations to be correct $85 \%$ of the time, a separate set of researchers believe the internal calibration of the Arsenator to be poor and have developed a correction algorithm addressing this problem (Sankararamakrishnan et al. 2008; Safarzadeh-Amiri et al. 2011). The nominal working range of the Arsenator spans from 5 to $100 \mu \mathrm{g} / \mathrm{L}$; but there is poor precision above 20 $\mu \mathrm{g} / \mathrm{L}$, due to saturation of the color sensor.

In recent times, there has also been a shift in the direction of research toward signal quantification by use of regular, day-to-day image producers. For example, Kearns et al. have used flatbed scanners to obtain digital images of the Hach EZ test kit; they then quantify the amount of arsenic detected by analysing the image using the computer software Colours (Kearns \& Tyson 2012). In this method, the image is deconstructed into its component RGB colour values and a calibration is developed between arsenic concentration and colour intensity. The authors report that the generation of their own arsenic calibration and the elimination of human colour subjectivity allows greater precision and reduces the frequency of false positive and false negative determinations. Similarly, Salman et al. have also used flatbed scanning to develop another arsenic detection method (Salman et al. 2012). Rather than working with an existing test kit, these authors have developed a Gutzeit method inspired spot test; they then analyse the colour intensity of the scanned image using a Visual Basic application. The method is reported to have a linear range of 2$20 \mu \mathrm{g} / \mathrm{L}$ of arsenic.

On-going efforts by colleagues in my own group aim to develop a MATLAB-based code for the calibration of arsenic determinations using the Hach EZ field kit. This particular research is rooted in the 
idea that increased portability can be afforded to the system by use of a digital camera, rather than a scanner, for the imaging of the test strip. One finding that has arisen from this work is that despite the ability of digital signal processing to increase precision of arsenic determinations, the analytical ability of the method continues to remain a function of the quality of the test kit itself. For example, manufacturing variability between individual test strips can have a significant effect on the reproducibility of field kit measurements. 


\subsection{ToWARds BetTer Arsenic Detection in WATER}

\subsubsection{The Ideal Arsenic Sensor}

While extensive research has been invested towards portable arsenic detection, current field techniques lack the robustness and reliability required to accurately declare a water source as being 'safe' or 'unsafe'. To be successfully used for mass monitoring of water drinkability, an ideal arsenic sensor must meet five essential criteria:

1) The arsenic sensor must be sensitive and selective.

Although the provisional MCL in most developing nations is $50 \mu \mathrm{g} / \mathrm{L}$, it is desirable to have the ability to measure down to the WHO limit of $10 \mu \mathrm{g} / \mathrm{L}$. It is important to quantitatively measure a range of arsenic concentrations to determine the extent of contamination. Moreover, since arsenic is a trace contaminant of water, most other potentially interfering species would be present in high excess. Therefore, the sensor must be selective to arsenic. Also, it is desirable to be able to differentiate the various species of arsenic, as the form in which it is present dictates its bioavailability and toxicity.

2) The arsenic assay must proceed quickly and yield reproducible results.

Millions of tube-wells need to be tested in regions such as Bangladesh; achieving this in practice requires an assay that can be performed in high throughput. Because most wells will be tested only once before being painted green or red, it is imperative that all sensors produce reliable and reproducible results.

3) The arsenic kit must be fully portable and robust enough for field use.

Preferably, the entire assay should be physically performed at the source location. Not only will this eliminate the need for complex sample labelling and handling, but more importantly it will increase local awareness about the monitoring process. For reliable use in the field, the sensor and all associated reagents/components must be robust enough to withstand harsh ambient conditions.

4) The arsenic detection process must reduce health and environmental risks.

The purpose of arsenic monitoring is to mitigate the development of arsenicosis and arsenic-related cancers within a population. Chemical processes in the analysis should reduce the risks of exposure by technicians and convert arsenic species to less toxic forms. Also, the testing of millions of tubewells will generate a large volume of chemical waste; the toxicity of these waste-products should be minimized such that they do not further poison the surrounding environment.

5) The arsenic monitoring plan must be affordable and easy to implement for the local population.

Current field kits average less than 1 USD per test (Safarzadeh-Amiri et al. 2011). This is a good target price for products designed for the developing world. To be affordably implemented, the ideal field sensor should be simple enough to be directly used by the well owners themselves, or local technicians, with only very minimal training. 


\subsubsection{The Merits of Microfluidics}

The field of microfluidics is characterised by the manipulation of small volumes of fluids, typically on the sub-millilitre scale (Martinez et al. 2010). Relative to their macro-scale counterparts, microfluidic processes have the advantages of faster reaction times and better process-control; reduced waste generation

and reagent consumption; system compactness and parallelization; and reduced cost and disposability (Whitesides 2006).

When compared to traditional analytical techniques, microfluidic processes are known for their general advantages associated with their smaller size. This smaller size is accompanied by many virtues such as portability, enhanced resolution, better process integration, and risk mitigation. Miniaturization allows smaller reaction volumes and diffusion distances, and therefore faster reaction times. In addition, such systems are capable of both high speed and high throughput processes. This quality is quite advantageous, because in many applications, information is of little value unless it can be generated quickly. Miniaturization also reduces costs. Smaller devices have lower material and waste disposal costs. This reduces the environmental footprint of the analysis. Faster reactions also have lower opportunity costs and personnel costs.

In light of these advantages, there have been a surge of microfluidic developments in the literature for the advancement of biomedicine. However, much of this research can also be adapted for environmental and other applications. The potential portability of microfluidics coupled with the successful employment of LOC devices in other fields readily lends this technology for the development of the ideal arsenic sensor outlined in the previous section. Several groups are currently exploring the use of microfluidics for the detection of arsenic and other heavy metals. 


\subsection{Candidate Methods For Microfluidic ArSenic Detection}

Given the challenges associated with reliable and affordable arsenic monitoring using current detection methods, much research has been devoted to developing alternate methods for arsenic detection. In the following sections, I highlight some of these candidate methods. I will compare the performance of these methods against the criteria for the ideal arsenic sensor outlined above, and comment on the methods' suitability for microfluidic adaptation. Table 3 organizes characteristics of each candidate arsenic detection method with regards to the criteria for an ideal sensor.

Table 3: General comparison of the performance attributes of alternate candidate methods for arsenic detection against the criteria for an ideal arsenic sensor, as outlined in section 3.1. Methods are evaluated on the basis of sensitivity and selectivity; speed and reproducibility; portability and robustness; possession of health and environmental risks; and affordability and ease of implementation.

\begin{tabular}{|c|c|c|c|c|c|c|}
\hline & \multicolumn{6}{|c|}{ Characteristics of an Ideal Arsenic Sensor } \\
\hline & $\begin{array}{l}\text { Sensitive and } \\
\text { Selective }\end{array}$ & $\begin{array}{c}\text { Quick and } \\
\text { Reproducible }\end{array}$ & $\begin{array}{c}\text { Portable and } \\
\text { Robust }\end{array}$ & $\begin{array}{c}\text { Low Health } \\
\text { and } \\
\text { Environmental } \\
\text { Risks } \\
\end{array}$ & $\begin{array}{l}\text { Affordable } \\
\text { and Easy }\end{array}$ & References \\
\hline \multicolumn{7}{|l|}{$\begin{array}{l}\text { Colorimetric } \\
\text { Methods }\end{array}$} \\
\hline $\begin{array}{l}\text { Molybdenum } \\
\text { Blue (PFI) }\end{array}$ & $\begin{array}{l}\text {-LOD: 1-15 } \\
\mu \mathrm{g} / \mathrm{L} \\
\text {-Selective over } \\
\mathrm{P}, \mathrm{Si}\end{array}$ & $\begin{array}{l}\text {-Detection } \\
\text { time: } \\
>30 \mathrm{~min} \\
\text {-Good } \\
\text { reproducibility }\end{array}$ & $\begin{array}{l}\text {-Not portable; } \\
\text { bulky } \\
\text { instrument }\end{array}$ & $\begin{array}{l}\text {-Toxic } \mathrm{AsH}_{3} \text { gas } \\
\text { generated }\end{array}$ & $\begin{array}{l}\text {-Expensive } \\
\text {-Requires some } \\
\text { specialized } \\
\text { skills }\end{array}$ & $\begin{array}{l}\text { (Linares \& } \\
\text { Castro 1986; } \\
\text { Rupasinghe et } \\
\text { al. 2004; } \\
\text { Rupasinghe et } \\
\text { al. 2001) }\end{array}$ \\
\hline $\begin{array}{l}\text { Molybdenum } \\
\text { Blue (pK } \\
\text { Effects) }\end{array}$ & $\begin{array}{l}\text {-LOD: } 4-8 \\
\mu \mathrm{g} / \mathrm{L} \\
- \text { Only } 20 \% \\
\text { accuracy at low } \\
\text { concentrations } \\
\text {-Selective over } \\
\mathrm{P}\end{array}$ & $\begin{array}{l}\text {-Detection } \\
\text { time: } \\
7-10 \text { min }\end{array}$ & $\begin{array}{l}\text {-Potentially } \\
\text { portable }\end{array}$ & $\begin{array}{l}\text {-Yes; As always } \\
\text { remains in } \\
\text { solution }\end{array}$ & -Yes & $\begin{array}{l}\text { (Dasgupta et } \\
\text { al. 2002; Dhar } \\
\text { et al. 2004) }\end{array}$ \\
\hline $\begin{array}{l}\text { Molybdenum } \\
\text { Blue (Ethyl } \\
\text { Violet) }\end{array}$ & $\begin{array}{l}\text {-LOD: } 10-25 \\
\mu \mathrm{g} / \mathrm{L} \\
\text {-Interference } \\
\text { from } \mathrm{P}, \mathrm{Si}, \mathrm{F}\end{array}$ & $\begin{array}{l}\text {-Detection } \\
\text { time: } \\
\sim 30 \text { min }\end{array}$ & $\begin{array}{l}\text {-Potentially } \\
\text { portable }\end{array}$ & $\begin{array}{l}\text {-Yes; As always } \\
\text { remains in } \\
\text { solution }\end{array}$ & -Yes & $\begin{array}{l}\text { (Morita \& } \\
\text { Kaneko 2006b; } \\
\text { Morita \& } \\
\text { Kaneko 2006a) }\end{array}$ \\
\hline Methylene Dye & $\begin{array}{l}\text {-LOD: } 10-100 \\
\mu \mathrm{g} / \mathrm{L}\end{array}$ & $\begin{array}{l}\text {-Detection } \\
\text { time: } \\
\sim 6 \text { min } \\
\text { (micelle } \\
\text { mediated) }\end{array}$ & $\begin{array}{l}\text {-Potentially } \\
\text { portable }\end{array}$ & $\begin{array}{l}\text {-Generation of } \\
\text { toxic } \mathrm{AsH}_{3} \text { gas; } \\
\text { but always } \\
\text { remains in } \\
\text { solution }\end{array}$ & -Yes & $\begin{array}{l}\text { (Ghosh et al. } \\
2002 ; \text { Kundu et } \\
\text { al. 2005; } \\
\text { Kundu et al. } \\
\text { 2002) }\end{array}$ \\
\hline $\begin{array}{l}\text { Sulfanilic Acid - } \\
\text { NEDA }\end{array}$ & $\begin{array}{l}\text {-LOD: } 18 \mu \mathrm{g} / \mathrm{L} \\
\text {-Selective over } \\
\text { P }\end{array}$ & $\begin{array}{l}\text {-Detection } \\
\text { time: } \\
\sim 30 \text { min }\end{array}$ & $\begin{array}{l}\text {-Portable } \\
\text {-Must prevent } \\
\text { exposure to air }\end{array}$ & $\begin{array}{l}\text {-Yes; As always } \\
\text { remains in } \\
\text { solution }\end{array}$ & -Yes & $\begin{array}{l}\text { Sharma et al. } \\
\text { 2011) }\end{array}$ \\
\hline
\end{tabular}

\section{Characteristics of an Ideal Arsenic Sensor}

\begin{tabular}{|c|c|c|c|c|c|c|}
\hline & \multicolumn{6}{|c|}{ Characteristics of an Ideal Arsenic Sensor } \\
\hline & $\begin{array}{l}\text { Sensitive and } \\
\text { Selective }\end{array}$ & $\begin{array}{c}\text { Quick and } \\
\text { Reproducible }\end{array}$ & $\begin{array}{c}\text { Portable and } \\
\text { Robust }\end{array}$ & $\begin{array}{c}\text { Low Health } \\
\text { and } \\
\text { Environmental } \\
\text { Risks } \\
\end{array}$ & $\begin{array}{l}\text { Affordable } \\
\text { and Easy }\end{array}$ & References \\
\hline \multicolumn{7}{|l|}{$\begin{array}{l}\text { Colorimetric } \\
\text { Methods }\end{array}$} \\
\hline $\begin{array}{l}\text { Molybdenum } \\
\text { Blue (PFI) }\end{array}$ & $\begin{array}{l}\text {-LOD: 1-15 } \\
\mu \mathrm{g} / \mathrm{L} \\
\text {-Selective over } \\
\mathrm{P}, \mathrm{Si}\end{array}$ & $\begin{array}{l}\text {-Detection } \\
\text { time: } \\
>30 \mathrm{~min} \\
\text {-Good } \\
\text { reproducibility }\end{array}$ & $\begin{array}{l}\text {-Not portable; } \\
\text { bulky } \\
\text { instrument }\end{array}$ & $\begin{array}{l}\text {-Toxic } \mathrm{AsH}_{3} \text { gas } \\
\text { generated }\end{array}$ & $\begin{array}{l}\text {-Expensive } \\
\text {-Requires some } \\
\text { specialized } \\
\text { skills }\end{array}$ & $\begin{array}{l}\text { (Linares \& } \\
\text { Castro 1986; } \\
\text { Rupasinghe et } \\
\text { al. 2004; } \\
\text { Rupasinghe et } \\
\text { al. 2001) }\end{array}$ \\
\hline $\begin{array}{l}\text { Molybdenum } \\
\text { Blue (pK } \\
\text { Effects) }\end{array}$ & $\begin{array}{l}\text {-LOD: } 4-8 \\
\mu \mathrm{g} / \mathrm{L} \\
- \text { Only } 20 \% \\
\text { accuracy at low } \\
\text { concentrations } \\
\text {-Selective over } \\
\mathrm{P}\end{array}$ & $\begin{array}{l}\text {-Detection } \\
\text { time: } \\
7-10 \text { min }\end{array}$ & $\begin{array}{l}\text {-Potentially } \\
\text { portable }\end{array}$ & $\begin{array}{l}\text {-Yes; As always } \\
\text { remains in } \\
\text { solution }\end{array}$ & -Yes & $\begin{array}{l}\text { (Dasgupta et } \\
\text { al. 2002; Dhar } \\
\text { et al. 2004) }\end{array}$ \\
\hline $\begin{array}{l}\text { Molybdenum } \\
\text { Blue (Ethyl } \\
\text { Violet) }\end{array}$ & $\begin{array}{l}\text {-LOD: } 10-25 \\
\mu \mathrm{g} / \mathrm{L} \\
\text {-Interference } \\
\text { from } \mathrm{P}, \mathrm{Si}, \mathrm{F}\end{array}$ & $\begin{array}{l}\text {-Detection } \\
\text { time: } \\
\sim 30 \text { min }\end{array}$ & $\begin{array}{l}\text {-Potentially } \\
\text { portable }\end{array}$ & $\begin{array}{l}\text {-Yes; As always } \\
\text { remains in } \\
\text { solution }\end{array}$ & -Yes & $\begin{array}{l}\text { (Morita \& } \\
\text { Kaneko 2006b; } \\
\text { Morita \& } \\
\text { Kaneko 2006a) }\end{array}$ \\
\hline Methylene Dye & $\begin{array}{l}\text {-LOD: } 10-100 \\
\mu \mathrm{g} / \mathrm{L}\end{array}$ & $\begin{array}{l}\text {-Detection } \\
\text { time: } \\
\sim 6 \text { min } \\
\text { (micelle } \\
\text { mediated) }\end{array}$ & $\begin{array}{l}\text {-Potentially } \\
\text { portable }\end{array}$ & $\begin{array}{l}\text {-Generation of } \\
\text { toxic } \mathrm{AsH}_{3} \text { gas; } \\
\text { but always } \\
\text { remains in } \\
\text { solution }\end{array}$ & -Yes & $\begin{array}{l}\text { (Ghosh et al. } \\
2002 ; \text { Kundu et } \\
\text { al. 2005; } \\
\text { Kundu et al. } \\
\text { 2002) }\end{array}$ \\
\hline $\begin{array}{l}\text { Sulfanilic Acid - } \\
\text { NEDA }\end{array}$ & $\begin{array}{l}\text {-LOD: } 18 \mu \mathrm{g} / \mathrm{L} \\
\text {-Selective over } \\
\text { P }\end{array}$ & $\begin{array}{l}\text {-Detection } \\
\text { time: } \\
\sim 30 \text { min }\end{array}$ & $\begin{array}{l}\text {-Portable } \\
\text {-Must prevent } \\
\text { exposure to air }\end{array}$ & $\begin{array}{l}\text {-Yes; As always } \\
\text { remains in } \\
\text { solution }\end{array}$ & -Yes & $\begin{array}{l}\text { Sharma et al. } \\
\text { 2011) }\end{array}$ \\
\hline
\end{tabular}




\begin{tabular}{|c|c|c|c|c|c|c|}
\hline Paper Based & $\begin{array}{l}\text {-LOD: } 1 \mu \mathrm{g} / \mathrm{L} \\
\text {-Selective over } \\
\mathrm{P}\end{array}$ & -Yes & -Yes & -Yes & -Yes & $\begin{array}{l}\text { (Nath et al. } \\
\text { 2014) }\end{array}$ \\
\hline \multicolumn{7}{|l|}{$\begin{array}{l}\text { Electrochemical } \\
\text { Methods }\end{array}$} \\
\hline ASV (Traditional) & $\begin{array}{l}\text {-Sensitivity on } \\
\text { the order of } \\
\text { ng/L } \\
\text {-Interference } \\
\text { from } \mathrm{Cu}\end{array}$ & $\begin{array}{l}\text {-Require } \\
\text { sample } \\
\text { pre-dilution } \\
\text { and electrode } \\
\text { surface } \\
\text { regeneration }\end{array}$ & $\begin{array}{l}\text {-Not robust; } \\
\text { electrodes are } \\
\text { fragile }\end{array}$ & $\begin{array}{l}\text {-Yes; As always } \\
\text { remains in } \\
\text { solution } \\
\text {-Require very } \\
\text { small volumes of } \\
\text { sample }\end{array}$ & $\begin{array}{l}\text {-Expensive to } \\
\text { fabricate } \\
\text { electrodes } \\
\text {-Relatively } \\
\text { simple } \\
\text { procedure }\end{array}$ & $\begin{array}{l}\text { (Luong et al. } \\
\text { 2007; Mays \& } \\
\text { Hussam 2009; } \\
\text { Feeney \& } \\
\text { Kounaves } \\
\text { 2002; Majid et } \\
\text { al. 2006) }\end{array}$ \\
\hline $\begin{array}{l}\text { ASV (Enzyme- } \\
\text { Modified) }\end{array}$ & $\begin{array}{l}\text {-Sensitivity on } \\
\text { the order of } \\
\mu \mathrm{g} / \mathrm{L} \\
\text {-Tolerant of } \mathrm{Cu}\end{array}$ & $\begin{array}{l}\text {-Detection time } \\
\text { function of } \\
\text { enzyme } \\
\text { metabolic rate } \\
\text {-Detection of } \\
\text { bioavailable As }\end{array}$ & $\begin{array}{l}\text {-Not robust; } \\
\text { electrodes are } \\
\text { fragile }\end{array}$ & $\begin{array}{l}\text {-Yes; As always } \\
\text { remains in } \\
\text { solution } \\
\text {-Require very } \\
\text { small volumes of } \\
\text { sample }\end{array}$ & $\begin{array}{l}\text {-Expensive to } \\
\text { fabricate } \\
\text { electrodes } \\
\text {-Relatively } \\
\text { simple } \\
\text { procedure }\end{array}$ & $\begin{array}{l}\text { (Male et al. } \\
\text { 2007; Cosnier } \\
\text { et al. 2006; } \\
\text { Stoytcheva et } \\
\text { al. 1998) }\end{array}$ \\
\hline SPE & $\begin{array}{l}\text {-Sensitivity on } \\
\text { the order of } \\
\mu \mathrm{g} / \mathrm{L} \\
\text {-Tolerant of } \mathrm{Cu}\end{array}$ & $\begin{array}{l}\text {-Detection time } \\
\text { function of } \\
\text { enzyme } \\
\text { metabolic rates } \\
\text {-Detection of } \\
\text { bioavailable As }\end{array}$ & -Yes & $\begin{array}{l}\text {-Yes; As always } \\
\text { remains in } \\
\text { solution } \\
\text {-Require very } \\
\text { small volumes of } \\
\text { sample }\end{array}$ & -Yes & $\begin{array}{l}\text { (Khairy et al. } \\
\text { 2010; } \\
\text { Sanllorente- } \\
\text { Méndez et al. } \\
\text { 2010) }\end{array}$ \\
\hline \multicolumn{7}{|l|}{$\begin{array}{l}\text { Biological } \\
\text { Methods }\end{array}$} \\
\hline Strip Based & $\begin{array}{l}\text {-Sensitivity on } \\
\text { the order of } \\
\mu \mathrm{g} / \mathrm{L} \\
\text {-Very selective } \\
\text { for As }\end{array}$ & $\begin{array}{l}\text {-Detection time } \\
\text { function of } \\
\text { enzyme } \\
\text { metabolic rates } \\
\text {-Detection of } \\
\text { bioavailable As }\end{array}$ & $\begin{array}{l}\text {-Strips are } \\
\text { portable } \\
\text {-Sensitive to } \\
\text { ambient } \\
\text { conditions }\end{array}$ & $\begin{array}{l}\text {-Yes; As always } \\
\text { remains in } \\
\text { solution } \\
\text {-Require very } \\
\text { small volumes of } \\
\text { sample }\end{array}$ & -Yes & $\begin{array}{l}\text { (Diesel et al. } \\
\text { 2009; Stocker } \\
\text { et al. 2003; } \\
\text { Aleksic et al. } \\
\text { 2007; Siegfried } \\
\text { et al. 2012) }\end{array}$ \\
\hline $\begin{array}{l}\text { Continuous Flow } \\
\text { Microfluidics }\end{array}$ & $\begin{array}{l}\text {-Sensitivity on } \\
\text { the order of } \\
\mu \mathrm{g} / \mathrm{L} \\
- \text { Very selective } \\
\text { for As }\end{array}$ & $\begin{array}{l}\text {-Detection of } \\
\text { bioavailable As } \\
\text {-Must } \\
\text { normalize } \\
\text { amount of } \\
\text { bacteria present }\end{array}$ & $\begin{array}{l}\text {-Potentially } \\
\text { portable } \\
\text {-Require } \\
\text { pumps, } \\
\text { microscopes, } \\
\text { refrigeration, } \\
\text { etc. } \\
\text { Sensitive to } \\
\text { ambient } \\
\text { conditions }\end{array}$ & $\begin{array}{l}\text {-Yes; As always } \\
\text { remains in } \\
\text { solution } \\
\text {-Require very } \\
\text { small volumes of } \\
\text { sample }\end{array}$ & $\begin{array}{l}\text {-Disposable } \\
\text {-Requires some } \\
\text { specialized } \\
\text { skills }\end{array}$ & $\begin{array}{l}\text { (Diesel et al. } \\
\text { 2009; Rothert } \\
\text { et al. 2005; } \\
\text { Theytaz et al. } \\
\text { 2009; Buffi et } \\
\text { al. 2011; } \\
\text { Merulla et al. } \\
\text { 2013) }\end{array}$ \\
\hline \multicolumn{7}{|l|}{$\begin{array}{l}\text { Electrophoretic } \\
\text { Methods }\end{array}$} \\
\hline ITP & $\begin{array}{l}\text {-Sensitivity on } \\
\text { the order of } \\
\mathrm{mg} / \mathrm{L} \\
\text { (Conductivity } \\
\text { detection) } \\
\text {-Selective over } \\
\text { Se }\end{array}$ & $\begin{array}{l}\text {-Detection } \\
\text { time: } \\
<10 \text { min }\end{array}$ & $\begin{array}{l}\text {-Potentially } \\
\text { portable } \\
\text {-Require } \\
\text { pumps, } \\
\text { microscopes, } \\
\text { etc. }\end{array}$ & $\begin{array}{l}\text {-Yes; As always } \\
\text { remains in } \\
\text { solution } \\
\text {-Require very } \\
\text { small volumes of } \\
\text { sample }\end{array}$ & $\begin{array}{l}\text {-Requires some } \\
\text { specialized } \\
\text { skills }\end{array}$ & $\begin{array}{l}\text { (Prest et al. } \\
\text { 2003; Prest et } \\
\text { al. 2005) }\end{array}$ \\
\hline
\end{tabular}




\begin{tabular}{|c|c|c|c|c|c|c|}
\hline & $\begin{array}{l}\text {-Interference } \\
\text { from } \mathrm{NO}_{3}^{-} \\
\mathrm{CO}_{3}{ }^{2-}, \mathrm{PO}_{4}{ }^{3-}\end{array}$ & & & & & \\
\hline $\mathrm{CE}$ & $\begin{array}{l}\text {-Sensitivity on } \\
\text { the order of } \\
\mu \mathrm{g} / \mathrm{L}(\mathrm{AFS} \\
\text { detection) } \\
\text {-As(III)/As(V) } \\
\text { resolution }\end{array}$ & $\begin{array}{l}\text {-Detection } \\
\text { time: } \\
<1 \text { min }\end{array}$ & $\begin{array}{l}\text {-Require } \\
\text { pumps, etc. } \\
\text {-AFS is not } \\
\text { portable }\end{array}$ & $\begin{array}{l}\text {-Yes; As always } \\
\text { remains in } \\
\text { solution } \\
\text {-Require very } \\
\text { small volumes of } \\
\text { sample }\end{array}$ & $\begin{array}{l}\text {-Expensive } \\
\text {-Requires some } \\
\text { specialized } \\
\text { skills }\end{array}$ & (Li et al. 2005) \\
\hline \multicolumn{7}{|c|}{$\begin{array}{l}\text { Surface Sensing } \\
\text { Methods }\end{array}$} \\
\hline SPR & $\begin{array}{l}\text {-LOD: } 1-15 \\
\mu \mathrm{g} / \mathrm{L} \\
- \text { Selective over } \\
\mathrm{P}\end{array}$ & -Yes & $\begin{array}{l}\text {-Potentially } \\
\text { portable } \\
\text {-Require } \\
\text { pumps, } \\
\text { microscopes, } \\
\text { etc. }\end{array}$ & $\begin{array}{l}\text {-Yes; As always } \\
\text { remains in } \\
\text { solution } \\
\text {-Require very } \\
\text { small volumes of } \\
\text { sample }\end{array}$ & $\begin{array}{l}\text {-Disposable } \\
\text {-Fabrication of } \\
\text { modified } \\
\text { surfaces is } \\
\text { difficult } \\
\text {-Requires some } \\
\text { specialized } \\
\text { skills }\end{array}$ & $\begin{array}{l}\text { (Wu, Zhan, et } \\
\text { al. 2012; Wu, } \\
\text { Liu, et al. } \\
\text { 2012; Forzani } \\
\text { et al. 2007) }\end{array}$ \\
\hline SERS & $\begin{array}{l}\text {-LOD: } 1 \mu \mathrm{g} / \mathrm{L} \\
\text {-Very selective } \\
\text { for As } \\
\text {-As(III)/As(V) } \\
\text { resolution }\end{array}$ & -Yes & $\begin{array}{l}\text {-Some Raman } \\
\text { spectrometers } \\
\text { are portable }\end{array}$ & $\begin{array}{l}\text {-Yes; As always } \\
\text { remains in } \\
\text { solution } \\
\text {-Require very } \\
\text { small volumes of } \\
\text { sample }\end{array}$ & $\begin{array}{l}\text {-Expensive } \\
\text {-Relatively } \\
\text { simple } \\
\text { procedure }\end{array}$ & $\begin{array}{l}\text { (Mulvihill et } \\
\text { al. 2008) }\end{array}$ \\
\hline \multicolumn{7}{|c|}{$\begin{array}{l}\text { Spectroscopic } \\
\text { Methods }\end{array}$} \\
\hline LIBS & $\begin{array}{l}\text {-LOD: } 100 \\
\mu \mathrm{g} / \mathrm{L}\end{array}$ & $\begin{array}{l}\text {-Must allow } \\
\text { time for As } \\
\text { adsorption to } \\
\text { substrate }\end{array}$ & $\begin{array}{l}\text {-Potentially } \\
\text { portable }\end{array}$ & $\begin{array}{l}\text {-Yes; do not } \\
\text { evolve toxic } \\
\mathrm{AsH}_{3} \text { gas }\end{array}$ & $\begin{array}{l}\text {-Very simple } \\
\text { setup }\end{array}$ & $\begin{array}{l}\text { (Haider et al. } \\
\text { 2014) }\end{array}$ \\
\hline XRF & -LOD: $0.7 \mu \mathrm{g} / \mathrm{L}$ & $\begin{array}{l}\text {-Must allow } \\
\text { time for As } \\
\text { adsorption to } \\
\text { substrate }\end{array}$ & $\begin{array}{l}\text {-Some XRF } \\
\text { detectors are } \\
\text { portable }\end{array}$ & $\begin{array}{l}\text {-Yes; do not } \\
\text { evolve toxic } \\
\mathrm{AsH}_{3} \text { gas }\end{array}$ & $\begin{array}{l}\text {-Very simple } \\
\text { setup }\end{array}$ & $\begin{array}{l}\text { (Sbarato \& } \\
\text { Sánchez 2001; } \\
\text { Barros et al. } \\
\text { 2010) }\end{array}$ \\
\hline $\mathrm{CL}$ & -LOD: $2 \mu \mathrm{g} / \mathrm{L}$ & $\begin{array}{l}\text {-Reaction time: } \\
\sim 1 \text { min } \\
\text {-Results agree } \\
\text { well with ICP } \\
\text { MS data }\end{array}$ & -Yes & $\begin{array}{l}\text {-Toxic } \mathrm{AsH}_{3} \text { gas } \\
\text { generated }\end{array}$ & $\begin{array}{l}\text {-Relatively } \\
\text { simple } \\
\text { procedure }\end{array}$ & $\begin{array}{l}\text { (Hashem et al. } \\
\text { 2011) }\end{array}$ \\
\hline
\end{tabular}

\subsubsection{Colorimetric Methods}

Colorimetric methods are desirable for portable arsenic monitoring because they have very simple detection. As with the traditional field kits, detection can be carried out by the human eye; or digital imaging can be used for more sophisticated analyses. An advantage of colorimetry with respect to field determinations is that several digital detection equipment are already easily portable; for example, a camera, a UV-Vis spectrometer, or a smartphone can be used as a digital detector. 


\subsubsection{Molybdenum Blue}

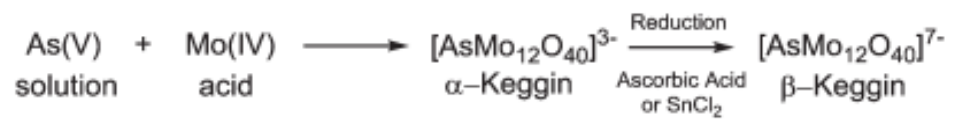

Figure 5: Basic scheme of the molybdenum blue reaction for arsenic detection (Barrows et al. 1985). Arsenic and molybdenum in solution react to form an $\alpha$-Keggin arsenomolybdate heteropolyacid, which, when reduced, forms a coloured $\beta$-Keggin product.

Like the Gutzeit method used by existing test strips, another colorimetric reaction for arsenic detection is the molybdenum blue assay (Levine et al. 1954). This assay consists of the reaction between arsenate and molybdenum to give a coloured heteropolyacid ion product (Figure 5). Conducting the molybdenum blue reaction essentially involves passive flows and mixing, so it is conceivable that this process can be microfluidically adapted. However, this chemistry faces interference from phosphates and silicates that compete with arsenic to react with molybdenum. Since phosphate and silicate levels of natural waters are typically much higher than the amount of arsenic present, they must be removed from solution before analysis.

One approach against such interference effects is to couple the molybdenum blue reaction with pervaporation flow injection (PFI) of the arsenic sample. PFI can be used to selectively remove arsenic out of solution in the form of arsine gas, for analysis; while phosphates and silicates are not volatilized and hence remain in solution (Linares \& Castro 1986; Rupasinghe et al. 2001; Rupasinghe et al. 2004). While these methods have approximate arsenic LODs in the range, $1-15 \mu \mathrm{g} / \mathrm{L}$; PFI is not suitable for portable adaptation due to the bulkiness of instrumentation, requirement for high temperatures, and complex sampling handing steps.

Another method to overcome phosphate interferences in this reaction is to take advantage of the different protonation states of penta- $\left(\mathrm{pK}_{\mathrm{a}} \sim 2.2\right)$ and trivalent $\left(\mathrm{pK}_{\mathrm{a}} \sim 9.2\right)$ species in solution (Dasgupta et al. 2002). Penta- and trivalent species can thus be separated and analysed independently (Dasgupta et al. 2002). The sample can also be assayed as two aliquots, once with all species in the pentavalent form and once with all species in the trivalent; the total arsenic content can be correlated to the difference of the two measurements (Dhar et al. 2004). Both of these approaches can detect arsenic with an LOD of approximately 4 to $8 \mu \mathrm{g} / \mathrm{L}$; an additional advantage being that ion separation has the potential to be carried out microfluidically using on-chip electrophoretic or chromatographic techniques. 

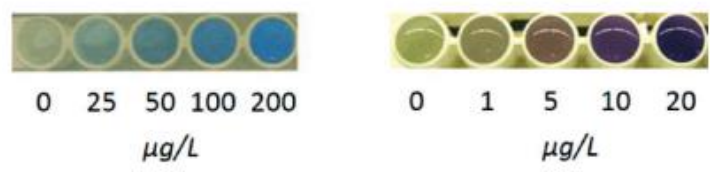

Figure 6: Visual detection of arsenic in solution using molybdenum chemistry. Left: Arsenic detection by formation of coloured micro particles via reaction of molybdoarsenates with ethyl violet dye (Morita \& Kaneko 2006a). Right: Ultra-sensitive arsenic detection via iodine-tetrachloride probing for molybdoarsenate micro particles (Morita \& Kaneko 2006b).

Due to the low molar absorptivity of molybdenum blue, arsenic detection by this reaction generally calls for spectrophotometric detection. Heteropoly blue has a low molar absorptivity $\left(1 \times 10^{-4} \mathrm{~L} / \mathrm{mol} / \mathrm{cm}\right)$ rendering direct quantification of the reaction by visual inspection quite difficult for environmental samples (Morita \& Kaneko 2006a). However, by complexing the heteropoly blue to an organic dye, Morita et al. have developed a molybdenum-based assay for arsenic detection by visual inspection (Morita \& Kaneko 2006a; Morita \& Kaneko 2006b). In this approach, interference effects are removed by anion exchange and masking agents; and then, following the classical reaction, the molybdenum blue product is induced to form stable, coloured micro particles by reaction with cationic ethyl violet dye. This method has a LOD of 25 $\mu \mathrm{g} / \mathrm{L}$ for arsenic. In a second reaction, Morita et al. use iodine tetrachloride as a 'probe' for the molybdoarsenate particles and achieve a final arsenic LOD of $10 \mu \mathrm{g} / \mathrm{L}$. Figure 6 shows the visual colour scales for these arsenic assays.

Okazaki et al. report a portable arsenic sensor, based on molybdenum blue chemistry, in which they employ a membrane filter to enrich the sensitivity of their assay (Okazaki et al. 2015). In a similar vein as Morita et al., the authors employ a cationic surfactant to form ion-associates with the generated molybdenum blue species; the technique has a reported arsenic LOD of $5 \mu \mathrm{g} / \mathrm{L}$, based on visual determination. Furthermore, the authors employ a $\mathrm{CaCO}_{3}$ cartridge system to perform sample workup procedures such as arsenic speciation, and phosphate interference removal. The cartridge-based sensor has been employed in preliminary field studies in both Bangladesh and Taiwan, and has proven to have adequate performance for groundwater analysis. However, I note that a substantial drawback of this proposed system with respect to routine groundwater analysis in affected regions is that the per-unit cost of $\mathrm{CaCO}_{3}$ far exceeds the target price range of 1-2 USD.

\subsubsection{Methylene Dye}

An alternate colorimetric reaction for arsenic detection is based on the direct interaction between arsenic and the cationic organic dye methylene blue (Ghosh et al. 2002; Kundu et al. 2005; Kundu et al. 2002). When reduced by arsine gas, methylene blue becomes colourless. The rate of reaction can be promoted when catalyzed within an anionic micelle in the presence of silver nanoparticles, which serve to 
facilitate the electron relay from arsine gas to the dye (Ghosh et al. 2002). The interaction of arsenic and methylene blue can also be facilitated through an As(V)-salicylic acid complex (Kundu et al. 2005). Here, the uni-negative ion complex binds methylene blue and can be quantitatively extracted out by toluene, while unbound dye remains behind in the aqueous phase. The blue-ness of the toluene extract is correlated with the amount of arsenic present in the original solution.

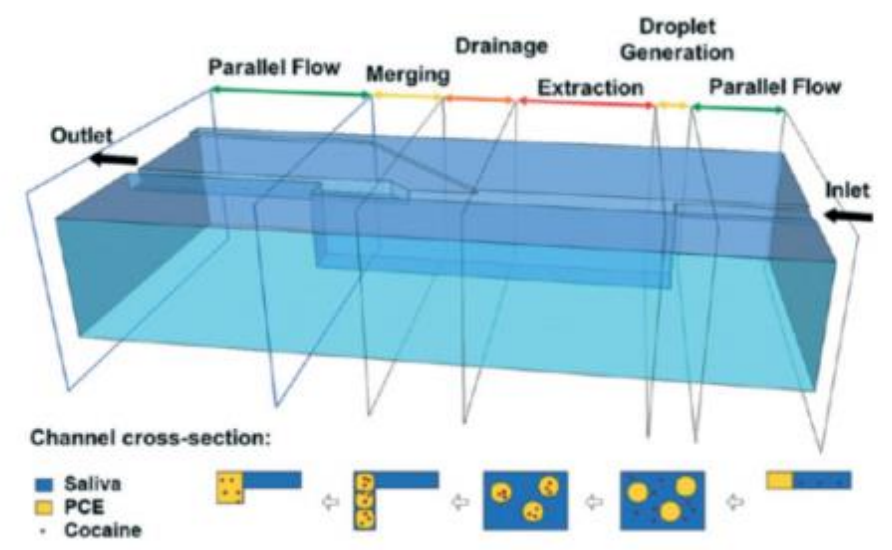

Figure 7: Schematic diagram of a microfluidic setup for on-chip liquid-liquid extraction of cocaine out of saliva. Top: 3D schematic diagram of a microfluidic chip (Wagli et al. 2013). Right to left: Cocaine containing saliva enters the chip and is introduced to droplets of the extraction solvent. Cocaine particles partition into the extraction media. The cocainecontaining droplets are then drained out of suspension and merged together. Bottom: Perpendicular cross-sections illustrating the extraction process along the length of the channel.

Unlike in traditional field kits, the arsine gas evolved by these methods remains in solution throughout the entire analysis, thereby reducing the risk of exposure to the technician. Further, microfluidic adaptations can be developed for both of these approaches. Typical microfluidic channel widths are on the order of $100 \mu \mathrm{m}$. This small size of microfluidic devices makes them suitable to manipulate the kinetics of nano-scale micelle formation. Also, with regards to the toluene extraction, a microfluidic liquid-liquid extraction protocol can be adapted from, for example, the work of Wagli et al. for cocaine extraction from saliva (Figure 7) (Wagli et al. 2013).

\subsubsection{Sulfanilic Acid-NEDA}

Another colorimetric method for arsenic detection is the reaction presented by Sharma et al. using sulfanilic acid and $N$-(1-naphtyl) ethylene diamine hydrochloride (NEDA) (Sharma et al. 2011). Here, arsenic (III) in solution first reduces the sulfanilic acid; the resultant product then goes on to react with NEDA to produce a magenta-coloured product. Sharma et al. carry out the entire process on disposable thin-layer chromatography (TLC) strip paptodes, and image the reaction using a colour scanner and MATLAB quantification of the detected RGB values. The total analysis time is 30 minutes; the method can 
detect arsenic in solution with a minimum LOD of $18 \mu \mathrm{g} / \mathrm{L}$. Advantages of this method are that paptode strips are readily portable, and the technique is free of phosphate interference because it is selective for trivalent species.

A microfluidic improvement of the paptode approach may result in improved reaction times by means of reduced diffusion distances and also active mixing. Since sulfanilic acid and NEDA do not react in the absence of arsenic, they can be premixed before on-chip mixing with arsenic. In these approaches, due to the three dimensional nature of microfluidic chips, image acquisition can no longer proceed via a scanner. Rather, a photograph of the chip may be obtained, and then analysed via similar MATLAB-type software signal quantification.

\subsubsection{Paper-Based Sensors}

Paper-based methods have been used in analytical detection for thousands of years. The first recorded history of paper-based colorimetric detection was in the $1^{\text {st }}$ century $\mathrm{AD}$, when Pliny the Elder used papyrus to estimate levels of Tyrian purple dyes in snails (Plinius Secondus (Pliny) n.d.). Currently dubbed microfluidic paper-based analytical devices ( $\mu$ PADs), this technology is popular today for its simplicity, portability, and low-cost mass production (Martinez et al. 2010).

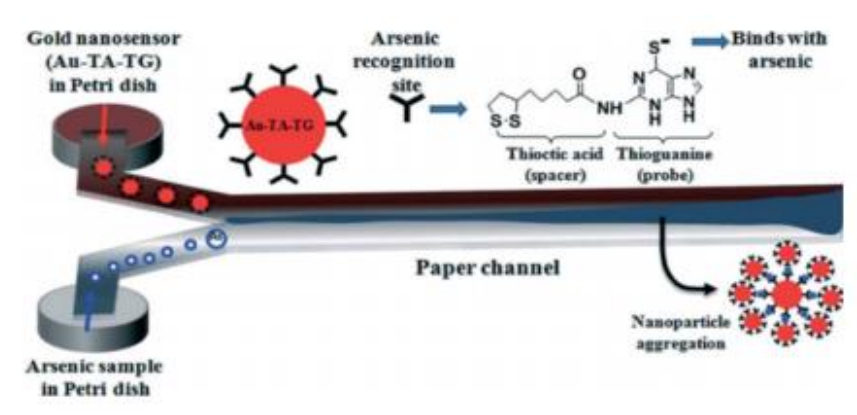

Figure 8: Schematic diagram of an ultrasensitive arsenic $\mu$ PAD (Nath et al. 2014). Arsenic containing solution and a modified gold nanosensor are allowed to wick up the arms of a Y-shaped chromatography paper device. Upon meeting at the junction, the two solutions react to form a dark, black-blue precipitate that is indicative of the presence of arsenic in the test sample. The amount of precipitate formed can then be related to the amount of arsenic originally present in solution

Nath et al. have developed a portable paper-based sensor for arsenic detection, by reaction with modified gold nanoparticles (Nath et al. 2014). Their rapid, ultrasensitive Y-shaped design is specific for As(III), and can detect arsenic concentrations down to $1 \mu \mathrm{g} / \mathrm{L}$. The authors propose the use of the modified gold nanosensor, Au-TA-TG, which binds to As(III) to produce a black-blue precipitate at the interface where the two species interact (Figure 8). In addition to low cost and portability, the technological 
advantage of having a paper substrate in this system is the facilitation of a slow, self-driven flow of the sample and nanosensor flows; which in turn allows for their reaction.

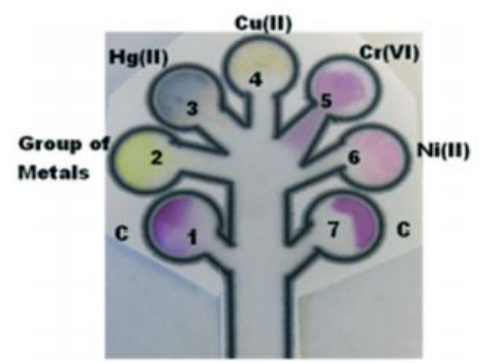

Figure 9: $\mathrm{A} \mu \mathrm{PAD}$ for the simultaneous detection of multiple metal ions in water (Hossain \& Brennan 2011). The device is fabricated by wax-printing of the channel design and inkjet printing of chromogenic agents onto locations 1-7. During analysis, contaminated water samples are introduced to the circular testing regions and the device is placed into a chromatography chamber; the chromogenic reagents are then moved into these regions by the upwards capillary action of deionized water in the base of the chamber.

Paper-based techniques are quickly gaining traction in heavy metal detection in both water and air samples (Hossain \& Brennan 2011; Mentele et al. 2012). For example, a patterned paper device has been developed for the simultaneous detection of several common metal ions in water (Figure 9) (Hossain \& Brennan 2011). Here, channels are delineated by a wax-printed pattern, and metal responsive reagents are inkjet deposited into defined zones within this pattern. During analysis, the water sample is added to the detection zone, and the device is placed upright into a chromatography chamber. The reaction proceeds as water is wicked up through the device by capillary action, and carries the deposited reagents to their respective reaction zones.

A particular advantage of this design is the ability to simultaneously test for the presence of several different metals. The different reaction zones may alternately also be used to generate a real-time calibration using known standards at the same time as the measurement of unknown samples. The simultaneous analysis of samples and standards together corrects for ambient variations. In later sections, I will further describe in why paper-based sensing may become a desirable route for arsenic well-testing.

\subsubsection{Electrochemical Methods}

Electrochemical detection methods have an inherent advantage over colorimetry with regards to miniaturization. The output of a colorimetric measurement is the absolute amount of an analyte in solution; so the smaller the sample size is, the more difficult the measurement. The output of an electrochemical measurement is the concentration of an analyte in the solution; this is independent of the size of the sample measured. In electrochemistry, smaller samples yield more accurate determinations due to higher surfacearea-to-volume ratios of the electrochemical probe, and lower interference effects from other species in 
solution. For this reason, there is a trend in analytical chemistry and arsenic sensing towards the development of miniaturised electroanalytical systems (Matysik 2003). Although electrochemical systems do not employ microfluidic flows in the classical sense, I have considered them in this review due to the benefits conferred to them by miniaturisation.

\subsubsection{Microelectrode Sensors}

There has been immense interest in the literature for the development of electrochemical techniques for arsenic detection; overwhelmingly, the method of choice has been anodic stripping voltammetry (ASV) (Baron et al. 2007; Dai et al. 2004; Feeney \& Kounaves 2000; Gao et al. 2013; Majid et al. 2006; Rajkumar et al. 2011; Xiao et al. 2008; Salimi et al. 2008; Dai \& Compton 2006). Arsenic detection by ASV has been studied for over 40 years (Forsberg et al. 1975). Broadly speaking, ASV analysis consists of two general steps: first, arsenic is preconcentrated onto the electrode surface via electrodeposition; and second, the plated metal is oxidized off the surface using an anodic linear potential sweep. For an in-depth review of electrochemical arsenic detection techniques, please refer to the works of Luong et al. and Mays et al (Luong et al. 2007; Mays \& Hussam 2009).

Typically, electrochemical detection techniques have very high sensitivities, with LODs on the order of $\mathrm{ng} / \mathrm{L}$ of arsenic in solution. Since arsenic contamination of water sources is typically on the order of $\mu \mathrm{g} / \mathrm{L}$, sometimes protocols call for the pre-dilution of real samples before analysis (Feeney \& Kounaves 2002). However, this introduces additional errors into the measurement.

When measuring arsenic, many electrochemical techniques suffer from copper interferences due to co-deposition of the two species on the electrode surface (Luong et al. 2007; Majid et al. 2006). To overcome this issue and increase arsenic selectivity, some groups have developed arsenic-respondent enzyme modified electrodes (Male et al. 2007; Cosnier et al. 2006; Stoytcheva et al. 1998). In these systems, the electrode measures enzyme activity, and this is correlated to the amount of arsenic present in solution. As with any biological application, a major drawback to this approach is the question of whether the enzyme activity is a true function of the total arsenic in solution, or if it measures only the bioavailable arsenic.

Although several microelectrodes and electrode arrays have been designed for arsenic detection, these devices are not suitable for implementation on the field. This is because electrochemical devices are inherently not robust. Electrode fragility and the need to regenerate the sensing surface between measurements render these systems ineffective outside of the controlled environment of a laboratory. Furthermore, typical electrodes consist of highly polished inert metal. Accordingly, they are expensive to fabricate and are not effective in disposable devices. 


\subsubsection{Screen Printed Electrodes}

One promising method that overcomes the challenges typically faced by electrochemical systems during arsenic detection is screen printed electrode (SPE) technology. Adapted from microelectronics, screen printing technology allows for the reproducible mass production of inexpensive single-use sensors (Renedo et al. 2007). SPE fabrication proceeds by inkjet printing of the electrode on top of a plastic or ceramic substrate. The versatility of this method stems from the ease of ink composition modifiability, which determines the sensitivity and selectivity of the electrode (Renedo et al. 2007). Arsenic (III)-specific SPEs have been fabricated using gold nanoparticle and acetylcholinesterase modified electrode inks; both methods are capable of determining the arsenic content of water with sub- $\mu \mathrm{g} / \mathrm{L}$ sensitivity, and are free of copper interferences (Khairy et al. 2010; Sanllorente-Méndez et al. 2010). These SPE designs show great promise for the future of portable in situ electrochemical arsenic detection. Furthermore, a three-electrode carbon, silver, silver/silver chloride arsenic SPE sensor has been developed paired with a portable handheld electrochemical analyser (Kim et al. 2013). This system can thus provide affordable, on-site, accurate, realtime measurements of arsenic content.

\subsubsection{Biological Methods}
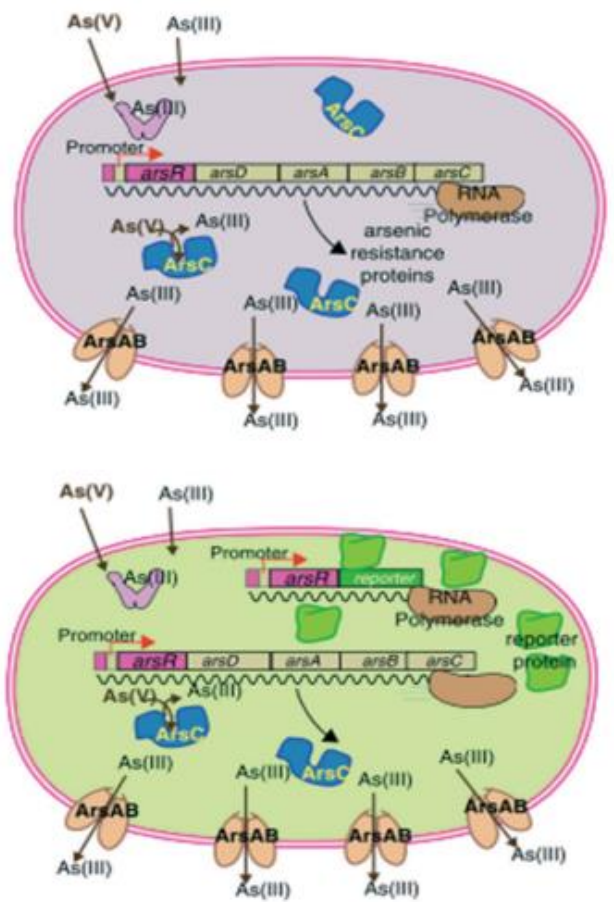

Figure 10: General mechanism of the arsR bacterial operon for arsenic detection (Diesel et al. 2009). Top: When arsenic is absent, the ArsR repressor protein binds the operator promoter site of the gene and prevents the transcription of arsenic defense genes ( $\operatorname{arsD}$, arsC, arsA, and arsB) further downstream. When arsenic is present, ArsR loses its affinity to the operator and no longer binds the promoter site; so RNA polymerase 
is able to transcribe the arsDCAB genes. ArsD is an additional regulatory protein, $\mathrm{Ars} C$ is an arsenate reductase that converts AsIJV) to AsIJIII); ArsAB is a membrane transporter that pumps AsIJIII) out of the cell. Bottom: Arsenic reporter strains have an additional gene construct of the arsR promoter region fused to a reporter gene. When arsenic is sensed by the cell, ArsR will unbind and the reporter gene will be transcribed and translated; detection of the reporter protein is an indication of the amount of arsenic that is sensed.

Biological detection methods developed for arsenic have been mostly based on the arsR bacterial operon. To easily measure the presence of arsenic, the bacteria are modified by plasmid transformation and the arsR operon is reconstructed to also include a reporter gene (Figure 10). Common reporter proteins include green fluorescent protein, luciferase, and $\beta$-galactosidase. Measurements of these arsenic-induced products allow LODs on the order of $1 \mu \mathrm{g} / \mathrm{L}$ of arsenic. A major advantage of biological sensing is high assay selectivity for the analyte of interest. For a detailed discussion of various bioassays for bacterial detection of arsenic, please refer to the 2009 review by Diesel et al (Diesel et al. 2009). I restrict my discussion below to focus only on those biological sensing systems that have been engineered portably.

\subsubsection{Portable Sensors}

For field applications, several biological sensors have been manufactured in a strip-based format. In one method, a paper-based colorimetric detector has been developed with $\mathrm{X}$-gal as substrate for a $\beta$ galactosidase reporter (Stocker et al. 2003). Here, paper strips are fabricated with bacterial cells pre-dried on their surface. During analysis, the strips are first incubated with arsenic-containing solution, and then Xgal is added and colour development is allowed to proceed. As the X-gal is digested by $\beta$-galactosidase, it loses its blue coloration. The magnitude of this change is related to the amount of arsenic present in solution. A similar $\beta$-galactosidase based arsenic sensor has also been developed with $\mathrm{pH}$ detection (Aleksic et al. 2007).

Furthermore, similar to the Arsenator, the ArsoLUX is a portable digital system for bioluminescence detection (Siegfried et al. 2012). During analysis, the arsenic water sample is introduced into a vial containing live, lyophilised bacteria which have the lux reporter gene; a luminosity measurement is then taken 10 seconds after sample introduction. The overall method is quick and sensitive for arsenic, but is accompanied by a start-up cost of 4,000 €. Field tests in Vietnam have shown that the ArsoLUX has performance comparable to the Arsenator and Merck field kits, but with considerably fewer steps and less reagent consumption (Siegfried et al. 2012). 


\subsubsection{Continuous-Flow Devices}

While strip-based kits are convenient for the field, increasingly bacterial detection has been made portable by traditional microfluidic implementation. There are now several examples in the literature of microfluidic geometries that have been designed for arsenic bioassays.
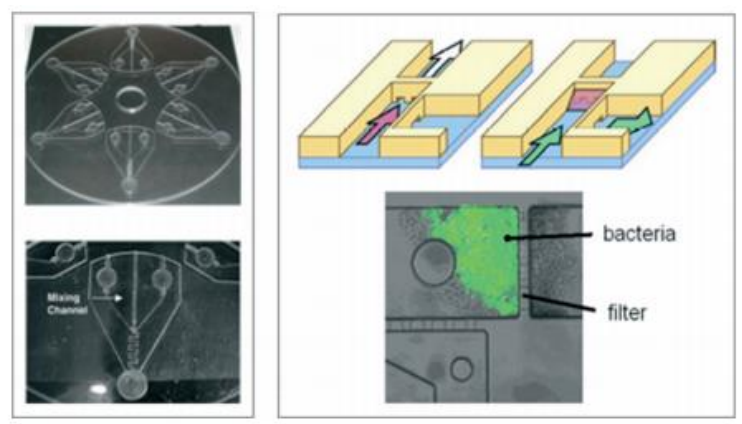

Figure 11: Two microfluidic geometries used in biological arsenic sensors. Left: Compact disc microfluidic arsenic sensor with six reaction zones; a close-up of one reaction zone is also shown (bottom) (Rothert et al. 2005). There are two reagent reservoirs at the inner region of the disc (one for the bacteria, and the other for the arsenic solution), a mixing channel with outward fluid flow driven by the centrifugal force, and a collection reservoir at the end of the channel for fluorescence detection. Right: Schematic of a microfluidic sensor with a bacteria filter (top), and a fluorescence image of bacteria that have sensed arsenic (bottom) (Theytaz et al. 2009).

A PMMA compact disk microfluidic biosensor has been designed by Rothert et al. to achieve sample flow and mixing by centrifugal force (Rothert et al. 2005). Typical microfluidic geometries require external pumps for sample introduction onto the chip. Here, on-chip sample introduction is achieved by the outward centrifugal force generated by spinning the disk-shaped design (Figure 11). After mixing, the bacterial GFP response is then detected by fluorescence microscopy. It is to be noted that use of a large enough product collection reservoir will further increase the portability of this system, by allowing for fluorescence imaging by a regular camera.

A more conventional example of a continuous flow microfluidic set-up consists of a closed, single use chip (Theytaz et al. 2009). Theytaz et al. propose such a channel geometry which includes a filter to immobilise bacteria while the arsenic solution flows through. The captured bacteria are then exposed to the arsenic solution for a fixed amount of time, and then the response is quantified. While the technique can identify a $50 \mu \mathrm{g} / \mathrm{L}$ arsenic threshold, it does not have adequate sensitivity to discern $10 \mu \mathrm{g} / \mathrm{L}$ of arsenic from a blank solution. As with the compact disk design, arsenic detection proceeds by whole cell bacterial sensing with a GFP reporter protein (Figure 11). 


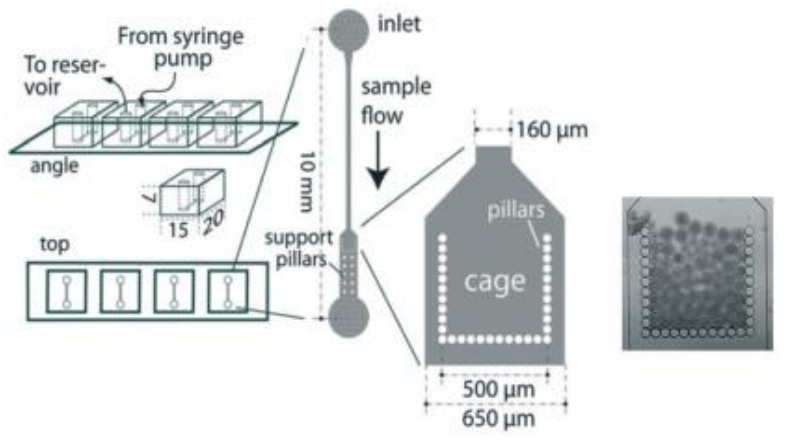

Figure 12: Schematic diagram of a microfluidic arsenic sensor with a cage for bacterial capture (Buffi et al. 2011). In the far right is an image of a microfluidic cage that has captured agarose beads containing bacteria.

In order to achieve signal reproducibility, it is imperative to use equal amounts of sensing bacteria for each measurement. To address this, microfluidic cartridges have been designed to consistently hold a constant amount of bacteria (Figure 12) (Buffi et al. 2011). Originally, these methods involved first encapsulating arsenic-responsive $E$. coli in $50 \mu \mathrm{m}$ diameter agarose beads, and then capturing these beads within a $500 \times 500 \mu \mathrm{m}$ microfluidic cage. The chips and beads are then exposed to arsenic solutions for detection. Signal reproducibility arises from the fact that the amount of bacteria in each bead and the number of beads trapped in each experiment are the same. The assay, based on the GFP reporter, can discriminate 10 and $50 \mu \mathrm{g} / \mathrm{L}$ from the blank with an incubation period of 1 hour. By reducing the spacing of the cage walls, applications of the microfluidic cage have been extended to also capture individual cells and bacterial spores for arsenic detection (Merulla et al. 2013). Recently, Truffer et al. have developed an automated, electronic sampling and monitoring device to quantitate the fluorescence signal from biosensors similar to those in Figure 12 (Truffer et al. 2014).

Bacterial metabolic rates are sensitive to ambient conditions, so it is not possible to normalise bioassay measurements taken at different times to a pre-calibrated curve. It is crucial that only those measurements taken under the exact same conditions are compared. As exemplified in the bioassay examples in Figure 11 and Figure 12, microfluidic devices can perform multiple determinations in parallel. Therefore, these geometries facilitate simultaneous analysis of a sample with replicates, or a sample with standard additions, or a sample with calibration standards.

Despite these advantages of bacterial microfluidic arsenic sensing, there are several inherent challenges associated with working with biological systems. A large issue is the maintenance of cellular activity and proliferation under ambient conditions. Biological responses are very sensitive to ambient stress factors such as heat, pressure, presence of oxygen, water availability, and nutrient availability. Although lyophilised and refrigerated cells can maintain their activity for prolonged periods, it is difficult 
and costly to maintain refrigeration of cells during transport and when working in the field. Another factor to consider is the total assay time. Unlike other diffusion-limited approaches, miniaturization of biological processes cannot significantly improve reaction times. The observed ArsR signal results from the cell detecting the presence of arsenic and then undergoing subsequent transcription and translation processes to produce a measurable protein product. This entire process is limited by the metabolic rate of the cell. Furthermore, as already mentioned, it is unknown whether the biological assay is measuring the total arsenic that is present in solution, or only the bioavailable amount.

\subsubsection{Electrophoretic Methods}

Electrophoresis is the movement of particles in solution under the influence of an electric field. Although it is most commonly used for the separation and purification of biological compounds such as nucleic acids and proteins; electrophoresis can be effectively used for the separation of any charged species in solution. In the 1990s, it was realised that the coupling of electrophoresis to microfluidics is quite advantageous for a number of reasons (Oh 1999). Importantly, microfluidic flows allow easy handling of small volumes and microfluidic chip separation avoids the need for long, tangled electrode wires. Furthermore, small scale microfluidic separation is much quicker than the long hours that are usually required for traditional electrophoretic techniques (Dorfman et al. 2013). Figure 13 illustrates two microfluidic geometries for electrophoretic detection of arsenic, using isotachophoresis and capillary electrophoresis.

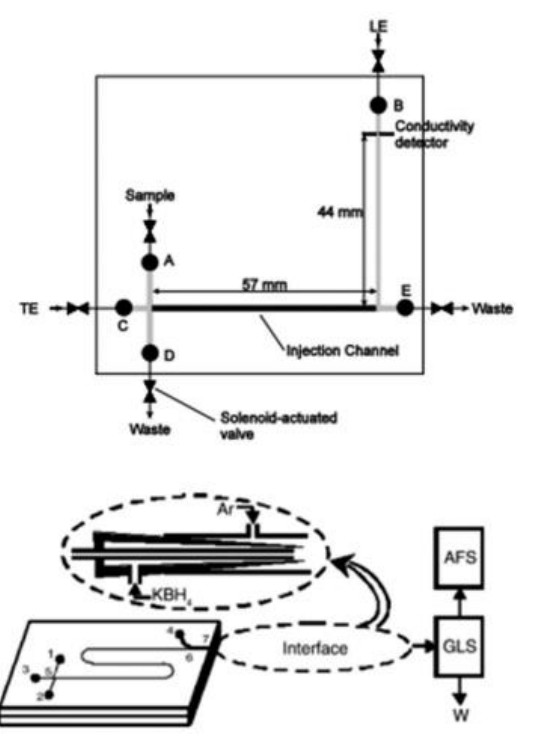

Figure 13: Schematic diagrams of microfluidic geometries for electrophoretic determination of arsenic. Top: Miniaturised separation device for isotachophoresis (ITP) with conductivity detection (Prest et al. 2005). Letters A-E refer to fluid inlets and outlets, and related valves. The arsenic containing sample is introduced as a plug between the leading and tailing electrolytes (LE, TE). Bottom: Chip-based capillary electrophoresis (CE) integrated with AFS detection (Li \& Lin 2009). Numbers 1-7 refer to fluid inlets and outlets, and reservoirs. The chip-to-AFS interface consists of a 'tube-in tube' design. 


\subsubsection{Isotachophoresis (ITP)}

Isotachophoresis (ITP) is an electrophoretic technique in which analytes are separated based on ionic mobility. The sample is introduced as a plug between a faster migrating leading electrolyte, and a slower migrating tailing electrolyte. Under an electric field, the different species present in the sample will migrate at different speeds based on ionic mobility and will separate into plugs; the length of each plug is in an indication of the amount of each species present. A common detection method for ITP separations is by conductivity measurements (Guijt et al. 2004). Microfluidic geometries have been developed for ITP separation and determination of arsenic compounds in conjunction with selenium (Figure 13) (Prest et al. 2003; Prest et al. 2005). However, these methods suffer from nitrate, carbonate, and phosphate interferences.

These methods also have very poor conductivity-based LODs that are on the order of $\mathrm{mg} / \mathrm{L}$ of arsenic in solution; this can potentially be improved microfluidically. In addition to sample separation, ITP systems can be used for sample focusing and pre-concentration. Notably, a shallow channel ITP- $\mu$ PAD, capable of up to 1,000-fold sample enrichment, has very recently been developed (Rosenfeld \& Bercovici 2014). Cheap, easy to manufacture, and easy to use, the wax printed device shows good promise for portable arsenic pre-concentration. Further, the technique may alleviate the current sensitivity barrier for conductivity detection.

\subsubsection{Capillary Electrophoresis $(\mathrm{CE})$}

Electrophoretic separations conducted using sub-millimeter or smaller capillaries are referred to as capillary electrophoresis (CE). To overcome the challenges associated with low sensitivities of conductivity-based detection, a CE system for arsenic sensing has been designed with downstream AFS detection (Figure 13) (Li et al. 2005). This apparatus consists of a microchip, a homemade interface with a 'tube-in-tube' design that reduces flow resistance while maintaining CE separation, and an AFS machine. In addition, a hydride-generation promoting makeup solution is used to transfer the sample to the AFS machine. This overall system has a As(III)/As(V) separation time of less than one minute.

While AFS is not a suitable field detection method, this CE system can potentially be employed on the field if coupled to a secondary assay downstream of the $\mathrm{As}(\mathrm{III}) / \mathrm{As}(\mathrm{V})$ separation location. For example, any of the portable arsenic sensors described in this review would work for this purpose. This ability to facilitate the integration of several different functional modules is an important advantage of microfluidic technology. Conceivably, the need for AFS analysis can be eliminated by coupling the separation to any of the secondary assays described in this review. 


\subsubsection{Surface Sensing Methods}

The electronic properties of metallic surfaces can be exploited in analytical processes to facilitate detection. Two such methods include surface plasmon resonance (SPR) and surfaced-enhanced Raman spectroscopy (SERS). Both of these techniques can be engineered as on chip processes; this microscale implementation lends SPR and SERS technology as favourable platforms for portable arsenic detection.

\subsubsection{Surface Plasmon Resonance (SPR)}

Surface plasmon resonance (SPR) allows for optical measurement of binding interactions between analytes and an immobilised molecule. SPR causes gold nanoparticles to appear red or blue depending on whether they are disperse or aggregate in solution (Xia et al. 2012). If the aggregation propensity of the nanoparticles is made to respond to arsenic, then this property can be used for arsenic detection.

Arsenic is strongly bound by sulphur-containing groups; a single arsenic atom can chelate up to three sulphurs at one time. This interaction has been exploited by chelation therapies for arsenicosis that use sulphur-containing agents (Weiner 2000). This chelation property has also been exploited for arsenic detection by use of gold nanoparticles that have surface modifications by sulphur-containing compounds (Kalluri et al. 2009). In the presence of As(III), the gold particles aggregate together due to chelation of the sulphur atoms in the surface groups, and the solution changes in colour from red to blue.

Furthermore, a label-free SPR detection method has been developed using the sulphur-containing phytochelatin-like peptide, $\mathrm{PC}_{3} \mathrm{R}$ (Xia et al. 2012). When unmodified gold nanoparticles and $\mathrm{PC}_{3} \mathrm{R}$ are mixed in the absence of arsenic, the gold is chelated by the peptide and aggregates are formed. When As(III) is present in the solution, it chelates the peptide and the nanoparticles become mono-dispersed; the solution changes in colour from blue to red. In addition to peptides, DNA aptamers can also be used for label-free gold nanoparticle assays for arsenic detection (Wu, Zhan, et al. 2012; Wu, Liu, et al. 2012).

With optical detection, gold nanoparticle-based arsenic assays have LODs as low as $0.015 \mu \mathrm{g} / \mathrm{L}$ and linear dynamic ranges spanning up to six orders of magnitude (Kalluri et al. 2009). While still powerful, these assays only have a visual LOD of about 5-15 $\mu \mathrm{g} / \mathrm{L}$. The SPR effect can also be used to look at binding interactions, as the resultant increase in mass causes a shift in the observed resonance angle.

Gold nanoparticles are very expensive. Thus, it is natural to use microfluidic geometries to perform these assays, as microfluidics require only small volumes of consumables. Commonly, SPR is carried out using flow injection (FI) geometries where a flow is used to transport the analyte in solution across the sensing surface. Microfluidics has proven to be a very suitable platform for this type of analysis. Some microfluidic SPR applications include molecular detection and biosensing, affinity analyses, and adsorption 
thermodynamics (Yang et al. 2005; Galopin et al. 2007; Luo et al. 2008; Kurita et al. 2006; Wang et al. 2007; Karlsson et al. 1991; Wei \& Latour 2008; Vernekar \& Latour 2005).

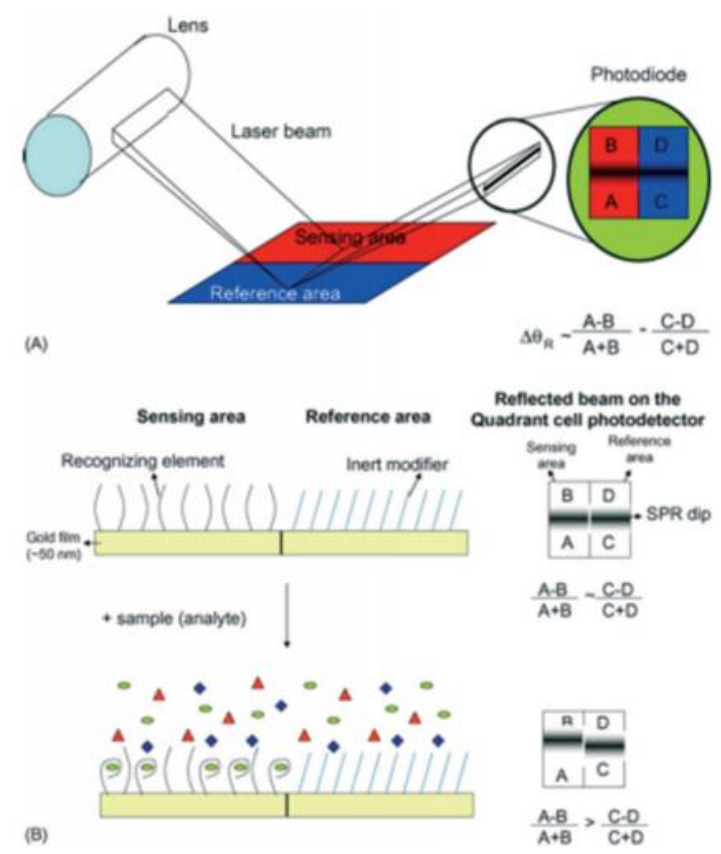

Figure 14: SPR sensor for arsenic detection (Forzani et al. 2007). A: Schematic of SPR sensor and incident laser beam. B: Arsenic-induced SPR shift generated by arsenic-sensing region relative to reference region.

As an adaptation of biotechnological microfluidic-SPR advancements towards an environmental application, Forzani et al. have developed a microfluidic SPR device for total arsenic detection from groundwater (Figure 14) (Forzani et al. 2007). Their differential SPR sensor is functionalised with an inert modifier as the reference surface and an arsenic-recognizing element as the sensing surface. Based on the SPR band shift between the two regions, the device can classify solutions as having more or less than 10 $\mu \mathrm{g} / \mathrm{L}$ of arsenic. The system can detect arsenic levels even less than $1 \mu \mathrm{g} / \mathrm{L}$ in pure buffer, but does not exhibit the same sensitivity in real samples due to matrix interferences.

\subsubsection{Surface-Enhanced Raman Spectroscopy (SERS)}

Surface-enhanced Raman spectroscopy (SERS) is a surface phenomenon that occurs from the local amplification of the electric field of a metal surface when its conduction band electrons resonate in phase with incident light. The first report of SERS for single molecule sensing was for the detection of single rhodamine 6G molecules. Since then, SERS has proven capable of probing various different single molecules for biomedical and other applications (Nie 1997; Qian \& Nie 2008).

Mulvihill et al. have taken this technology back to its original application of inorganic molecular detection through the development of a SERS sensor for trace arsenic analysis (Mulvihill et al. 2008). This 
sensor consists of a dense array of silver nanoparticle monolayers modified with adsorbed poly(vinyl pyrrolidone) polymer (PVP), which stabilizes the nanocrystal structure and facilitates interactions between silver and arsenate. Arsenic sensing experiments are carried out by bringing a droplet of the arsenic solution in contact with the sensing surface, and covering with a glass cover slip; quantitation is primarily based on the detection of the As-O Raman stretch, with a linear response observed from $1-180 \mu \mathrm{g} / \mathrm{L}$ of arsenic in solution. Since SERS spectra provide a chemical 'fingerprint' of the sample, this method can also differentiate between $\mathrm{As}(\mathrm{III})$ and $\mathrm{As}(\mathrm{V})$ in solution.

During static SERS measurements, it is necessary to search for SERS 'hot spots' which have measurably high signals; it has been shown that the use of microfluidic geometries can resolve this problem (Strehle et al. 2007). Microfluidic SERS sensors have been used for a variety of applications, including high efficiency target molecule detection, drug detection, and bacterial stain discrimination, among others (Xu et al. 2011; Ackermann et al. 2007; Walter et al. 2011). Such devices would likely confer similar benefits to the removal of SERS hotspots during arsenic analysis as well.

\subsubsection{Spectroscopic Methods}

Environmental chemistry often involves the spectroscopic analysis of solid matrices; laser induced breakdown spectroscopy (LIBS) and X-ray fluorescence (XRF) are two solid-state analysis techniques that have currently been explored for arsenic detection. Chemiluminescence (CL) is another spectroscopic technique which, however, detects arsenic in its gas phase.

\subsubsection{Laser Induced Breakdown Spectroscopy (LIBS}

Laser induced breakdown spectroscopy (LIBS) is a subset of AES that specifically uses energy from a laser pulse as the excitation source. While literature exists on the use of LIBS for the analysis of solid, liquid, and gas state samples, the analysis of solids is most popular (Rusak et al. 1997). However, LIBS analysis of water samples can be carried out by use of a wood substrate (Chen et al. 2010). The main advantage of LIBS is that the entire setup is quite simple, consisting of only a neodymium-doped yttrium aluminium garnet (Nd-YAG) laser, a high sensitivity spectrometer, and a computer for data acquisition (Theriault et al. 1998; Marquardt et al. 1996). 


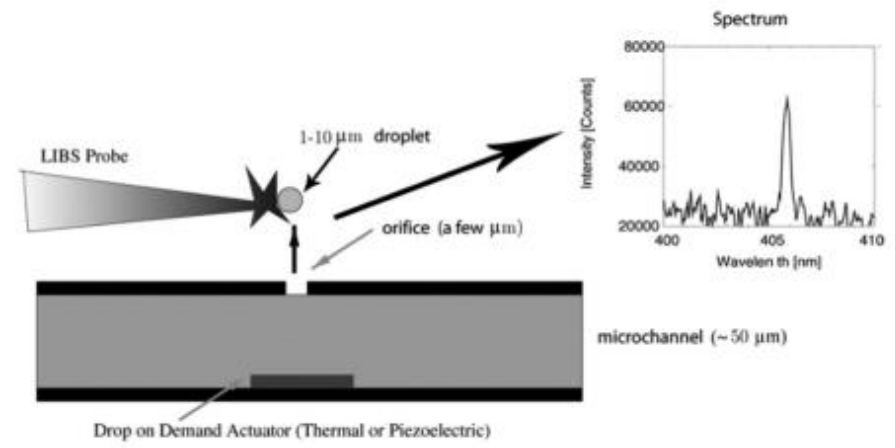

Figure 15: Conceptual layout of a potential LOC set-up for a $\mu$ LIBS system (Y. Godwal et al. 2008). Micron-sized sample droplets are first generated using a thermal or piezoelectric technique, and then their chemical composition is analysed by a LIBS probe. We note that the flow-through nature of the system renders it a suitable detector for electrophoretic separation techniques such as ITP and CE. Therefore, $\mu$ LIBS may prove to be a more practical choice over conductivity or AFS detection for portable arsenic monitoring.

LIBS has been used to detect arsenic in solution at concentrations down to $100 \mu \mathrm{g} / \mathrm{L}$ (Haider et al. 2014). This sensitivity has been achieved through concentration enhancement via boiling, followed by sample adsorption onto a zinc oxide substrate. However, miniaturised LIBS systems ( $\mu$ LIBS) have been developed for the elemental analyses of sodium and lead, which achieve similar sensitivities while analysing liquid samples directly (Yogesh Godwal et al. 2008; Y. Godwal et al. 2008). $\mu$ LIBS applications require pulse energies on the order of $100 \mu \mathrm{J}$; so they are capable of analysing smaller spot areas and also are compatible with fibre lasers. Such features facilitate the possibility of LOC-type LIBS technology. A $\mu$ LIBS system will likely have increased sensitivity for arsenic relative to the existing method, and will also likely be a suitable method of detection for coupling with electrophoretic separations (Figure 15).

\subsubsection{X-ray Fluorescence (XRF)}

$\mathrm{X}$-ray fluorescence (XRF analysis is based on the detection of the characteristic fluorescent lines that result from the irradiation of a sample with X-ray light. This technique is non-destructive, and is theoretically capable of the detection of atoms in any state of matter. In practice, as with LIBS, XRF detection is limited to solid state samples because denser samples absorb more radiation and therefore produce a larger measurable signal (Brouwer 2010).

XRF has been used for the analysis of arsenic in water samples by pre-concentration of the arsenic on to solid substrates such as tape and alumina (Sbarato \& Sánchez 2001; Barros et al. 2010). The latter approach, in which arsenic is pre-concentrated onto alumina particles and then centrifuged into pellets for solid-state analysis, has an LOD of $0.7 \mu \mathrm{g} / \mathrm{L}$ of arsenic in solution. In microfluidics, it is possible to concentrate very high levels of arsenic into very small areas; this will likely increase the sensitivity of such 
a method and eliminate the need for centrifugation. As an additional advantage to field sensing, some XRF detectors are currently already portable.

\subsubsection{Chemiluminescence $(C L)$}

Chemiluminescence (CL) is the generation of light as a result of a chemical reaction. The CL generated from the reaction of arsenic and ozone $\left(\mathrm{O}_{3}\right)$ has been known for more than 30 years (Fujiwara et al. 1982). Furthermore, both arsine gas and ozone have distinct CL spectra. During CL analysis, arsenic is first converted to arsine gas via hydride generation, and then the resultant arsine gas is introduced via a carrier gas flow to an ozone chamber for further reaction. This method can detect arsenic levels on the order of $1 \mu \mathrm{g} / \mathrm{L}$, but is not very amenable to the field due to the requirement of a carrier gas tank (Stedman et al. 1983).

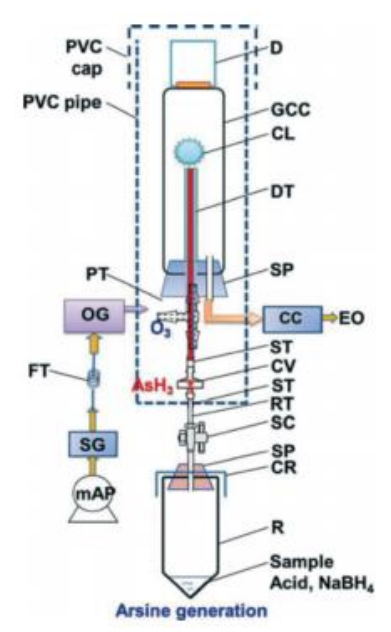

Figure 16: Schematic set-up of a portable gas-phase CL system for arsenic detection (Hashem et al. 2011). The set-up consists of two fundamental regions: an arsine generator (bottom), and a gas-phase CL detector (top). Arsine generation occurs in a $50 \mathrm{~mL}$ vial, and proceeds via $\mathrm{NaBH}_{4}$ reduction of arsenic in solution. The evolved gas flows through a stopcock to the detector, where it reacts with ozone to generate CL. Signal detection occurs with $1 \mu \mathrm{g} \mathrm{L}-1$ resolution by use of a photomultiplier tube connected to the CL cell by an optical window.

Hashem et al. have developed a portable set-up for arsenic CL that avoids the need for a carrier gas, or liquid reagents, altogether (Figure 16) (Hashem et al. 2011). In this approach, arsenic in solution is converted to arsine gas by means of a solid reducing agent; and ozone is generated separately using a corona discharge source. A pressure difference then drives the arsine gas into the ozone chamber, and the resultant $\mathrm{CL}$ is detected using a photomultiplier tube (PMT). The entire process has a reaction time of one minute, and an arsenic LOD of $2 \mu \mathrm{g} / \mathrm{L}$. The system is small, lightweight, and has low power consumption; accordingly, it has strong potential to perform well on the field. However, both arsine gas and ozone are toxic to human health, so this may not be an ideal method when considered from a use-safety standpoint. 
We note that arsenic CL has recently also been used in a slightly different application to detect the amount of arsenic, and other heavy metals, removed from water using a nanoporous anodic aluminium oxide (AAO) membrane (Chang et al. 2014). Here, the nanomembrane is affixed on a microfluidic platform, and pneumatic and centrifugal microfluidic forces are used for the filtration of the sample as well as the CL detection of the signal. Furthermore, another microfluidic CL system has also been developed for As(III)/As(V) speciation (Taokaenchan et al. 2014). This system, capable of detecting arsenic levels below $10 \mu \mathrm{g} / \mathrm{L}$, splits the injected sample into two flows, and independently detects the two arsenic species by taking advantage of the fact that $\mathrm{As}(\mathrm{III})$ and $\mathrm{As}(\mathrm{V})$ undergo different $\mathrm{CL}$ reactions. 


\subsection{Micro Total Analytical Systems ( $\mu$ TASs) FOR Arsenic Detection}

The arsenic detection process can be broken down into three stages: sample preparation, sample analysis, and signal processing. Above, I have outlined potential microfluidic LOC techniques that address the issue of arsenic sample analysis. Oftentimes these labs-on-a-chip require labs-around-the-chip to perform pre- and post- analysis processing. By the term labs-around-a-chip, I am referring to the pumps, microscopes, and other large scale external instrumentation that are required for the use and function of microfluidic LOCs.

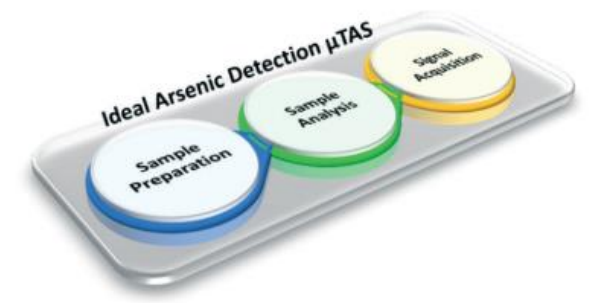

Figure 17: Schematic diagram of the ideal $\mu$ TAS for portable arsenic detection. Here, the entire arsenic detection process is carried out by a single integrated system. The first step, sample preparation, involves sample introduction to the chip, any arsenic pre-concentration and speciation conversions, as well as interference masking. The next step, sample analysis, involves the actual arsenic assay of choice. And the final step, signal acquisition, involves any additionally required detection processes. Microfluidic techniques do currently exist which perform each of these three detection stages separately.

As described by Manz et al., a total analytical system carries out "sampling, sample transport, any necessary chemical reactions, chromatographic separations, as well as detection "(Manz et al. 1990). A micro total analysis system ( $\mu$ TAS) would encompass an integration of all of these processes into a single platform. The ideal arsenic sensor, according to my definition, is a $\mu$ TAS that can achieve the entire arsenic detection process, starting with well water on-site, from start to finish (Figure 17). General advances in microfluidics technology can serve to fulfil the needs to make this a reality; a few of these advances, relating to pre- and post- arsenic analysis processes, are highlighted below.

\subsubsection{Specific Concerns for Arsenic Analysis}

A particular challenge concerning the analysis of real water samples is interference effects and arsenic oxidation requirements. These issues must be addressed before the arsenic detection assay can proceed. Arsenic and phosphorus compounds share very similar chemistry, owing to their similarities in size and shape. A simple method for the removal of phosphate interference from arsenic containing solutions is by ion chromatography. Ion chromatography for phosphate and arsenate separation proceeds via anion exchange. Phosphorus(V) and arsenic(V) species have different $\mathrm{pK}_{\mathrm{a}} \mathrm{s}$ ( 2.2) from arsenic(III) species ( 9.2). By starting with an initial solution in which all arsenic is reduced to arsenic(III), ion 
chromatography can then be used to separate the arsenate and phosphate compounds in solution (Dasgupta et al. 2002).

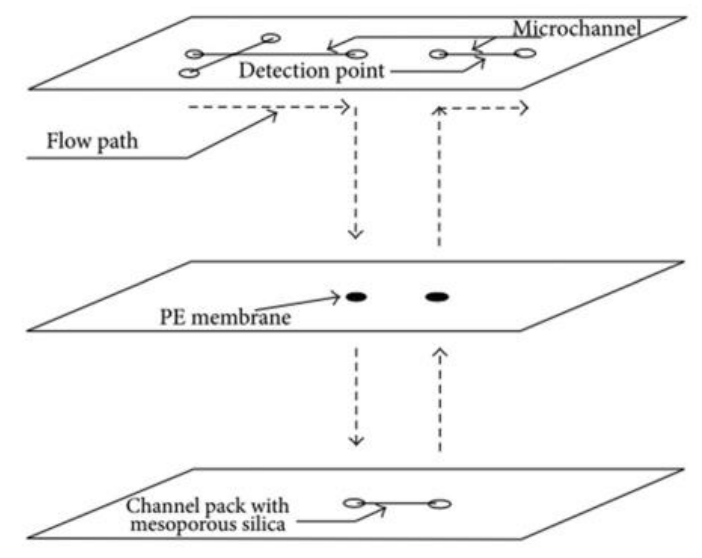

Figure 18: Schematic diagram of a microfluidic size-exclusion liquid chromatography (LC) system (Chan et al. 2014). A packed, mesoporous silica column is interfaced to a poly-dimethyl siloxane (PDMS) microfluidic chip by means of a polyethylene (PE) membrane. Using just the pressure generated by a standard syringe pump, the device can size-exclusion separate dye molecules from biopolymer mixtures.

There are several examples in the literature regarding microfluidic chromatography (Gomez 2011). As a proof-of-principle, an on-chip liquid chromatography (LC) system has been developed for the separation of dye and biopolymer mixtures (Figure 18) (Chan et al. 2014). In this design, a microfluidic column, which is packed with mesoporous silica beads, separates dyes from biopolymers using only the pressure generated by a standard syringe pump. Using soft lithography, it is possible to fabricate a multilayered poly-dimethyl siloxane (PDMS) chip that is interfaced via a polyethylene (PE) membrane to a silica packed channel. It is conceivable that similar packed column devices can be designed for the analysis of environmental samples as well; in the instance of ion exchange channels the mesoporous beads must be replaced with charged polymers instead.

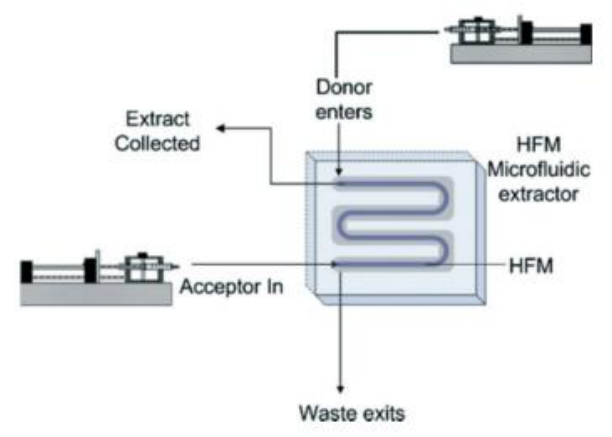

Figure 19: A microfluidic hollow fibre membrane extractor for arsenic pre-concentration (Hylton \& Mitra 2008). Supported liquid membrane extraction (SLME) occurs of arsenic from the original aqueous donor solution (introduced in the microfluidic channel) into an organic acceptor solution (introduced within the hollow fibre membrane, HFM); the pHs of the donor and acceptor solutions are adjusted such that arsenic is uncharged in solution and preferentially partitions into the organic phase. 
A common drawback of miniaturisation with regards to trace analysis is that it is difficult to detect low levels of an analyte from a very small sample volume. With regards to arsenic detection, this can potentially be addressed via microfluidic enrichment and pre-concentration. A microfluidic extractor has been developed for the on-chip concentration of arsenic within a hollow-fibre (Figure 19) (Hylton \& Mitra 2008).

Iron oxide binding of arsenic is a fairly well-understood phenomenon that is commonly used in industrial waste management and pollution control systems for arsenic removal (Katsoyiannis \& Zouboulis 2002; Thirunavukkarasu et al. 2003; Mayo et al. 2007; Yavuz et al. 2010; Yean \& Cong 2005; Kurniawan et al. 2012). Iron oxide binding can therefore be another potentially viable option for arsenic preconcentration applications. Iron oxide pre-concentration binding of arsenic can easily be achieved using a microfluidic platform. As previously mentioned, microfluidic systems readily facilitate diffusion-controlled reactions such as adhesion binding. It can be conceived that arsenic and iron oxide solutions can be mixed on chip. After a characteristic binding time, the arsenic-containing particles can be collected and the arsenic can be re-suspended in a smaller volume for further analysis. Past research efforts in my own group has focused on the magnetic pull down and desorption of arsenic from iron-oxide magnetic particles. This work was motivated by the idea that the arsenic-containing extract can then be microfluidically analysed further downstream.

\subsubsection{General Issues of Portability}

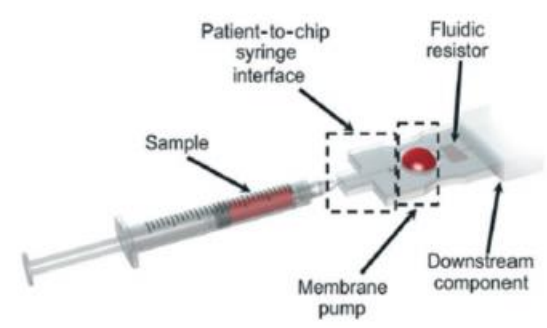

Figure 20: Schematic diagram of a hand-powered microfluidic membrane pump (M. M Gong et al. 2012). The sample is first introduced via syringe, through the syringe interface, to fill and inflate the membrane pump. Then, the membrane pump deflates when pressed and pushes the sample flow through the fluidic resistor to the downstream component of the chip. The rate of fluid flow is regulated by the properties of the fluidic resistor, not the pressure exerted by the mechanical pressing force

Some more general issues for portable continuous-flow microfluidics are sample introduction onto the device, and visualisation of micro-scale processes. Traditionally, microfluidic sample introduction occurs via external syringe pumps; but these pumps cannot be brought to the field. Alternative micro-pumps have been developed with various modes of sample actuation, but many still have limited portability due to continuous need for a power supply. However, hand-powered pumps are one mode of sample 
introduction that do not require any additional resources. For example, in the hand-powered microfluidic membrane pump developed by Gong et al., the sample is first injected via syringe into the membrane pump, causing the membrane to deform; and then subsequent deflation of the pump by the operator causes the sample to travel downstream (Figure 20) (Max M Gong et al. 2012).

Also, the traditional inverted microscopes and cameras typically used to magnify and capture images of microfluidic devices are extremely expensive, and not practical for use on the field. However, recently, the 'Foldscope', an origami-based paper microscope, has been constructed for a mere fifty cents (Cybulski et al. 2014). This device consists simply of a sheet of punched cardstock, a spherical lens, a lightemitting diode (LED) with a diffuser panel, and a battery to power the diode. It is easily assembled via folding, weighs only 8 ounces so is easily portable, and yet has a magnification power of up to 2,000 times. In addition to the Foldscope, there is also much interest in the use of smartphones coupled to magnifying lenses as portable imaging detectors (Park et al. 2013; Shen et al. 2012; Zhu et al. 2011; Martinez et al. 2008; García et al. 2011; Selck et al. 2013; Lu et al. 2009; Ruano-López et al. 2009).

Possibly, the most useful development forward towards increasing the accessibility of portable arsenic testing to the developing world is via signal quantification by use of cell phones. The computational power of the modern smartphone rivals that of computers; so smartphones can be used both for image acquisition, via camera, and also for image processing, via a specialized mobile application. This, coupled to the fact that these devices are becoming increasingly popular everywhere around the world, renders smartphone technology very favourable for portable microfluidic process visualisation. For example, Sicard et al. have integrated the hardware, software, and social media capabilities of cellphones to develop a water quality monitoring system (Sicard et al. 2015). Here, a paper-based water quality sensor is imaged by a cell phone and the signal is quantified by a downloadable image processing application; the results are then mapped onto an online water monitoring network. The implementation of such a system for arsenic well monitoring and mapping may generate a paradigm shift in how the issue of arsenic contamination is tackled both in Bangladesh and around the world. 


\subsection{SUMMARY}

Arsenic contamination is an ubiquitous problem all over the world; particularly in Bangladesh, where contamination is attributed to naturally high arsenic levels in ground sediments (WHO 2001; Petrusevski \& Sharma 2007; Cicero 2009). Although the current WHO MCL for arsenic in drinking water is set at $10 \mu \mathrm{g} / \mathrm{L}$, analytical and economic constraints cause Bangladesh and many other developing nations to adopt a higher limit of $50 \mu \mathrm{g} / \mathrm{L}$ (Petrusevski \& Sharma 2007; Smith et al. 2000; Flanagan et al. 2012). Unfortunately, it is expected that 1 out of 100 people who consume water in excess of $50 \mu \mathrm{g} / \mathrm{L}$ of arsenic, will die due to an arsenic-related cancer (WHO 2001).

To date, 6-11 million tube-wells need to be tested for arsenic contamination in Bangladesh (Jakariya et al. 2007). While common laboratory techniques such as AAS, MS, and AFS all have the ability to detect arsenic levels well below the WHO limit, their high costs and the requirement for a centralized facility render them ineffective for mass monitoring applications. Furthermore, the portable Gutzeit-method based arsenic test strips introduced in the 2000s have been shown to lack the safety, sensitivity, and reliability required for a human-health risk determination.

An ideal arsenic sensor, aimed to address the global need for arsenic monitoring, must meet the criteria of sensitivity and selectivity, speed and reproducibility, portability and robustness, health and environmental safety, and affordability and ease of use. Several efforts are currently in place for the development of alternate arsenic sensors using colorimetric, electrochemical, biological, electrophoretic, surface sensing, spectroscopic and paper-based methods. The integration of microfluidic technology lends many advantages to point-of-care-type device development; including increased portability, faster reactions, higher throughput, increased reliability, reduced cost, reduced health and environmental impacts, and easier handling.

Presently, I have reviewed these different alternate detection methods in terms of their potential as a routine arsenic sensor. Many of the mentioned alternate arsenic analyses either already have been, or can be, implemented microfluidically in the form of LOC devices; it is also evident that many sample work-up and other required manipulations can be portably implemented as well. In light of these developments, it is clear that the future of portable microfluidic arsenic detection is quite bright. Once an integrated LOC is developed for arsenic analysis, all that is required is to click together the necessary auxiliary modules and voila: the ideal portable arsenic sensor. 


\section{Development of a Portable Platform for Arsenic DETECTION}

\subsection{INTRODUCTION}

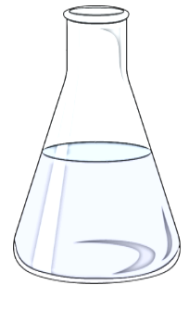

Assay

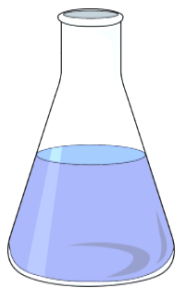

Colour Change

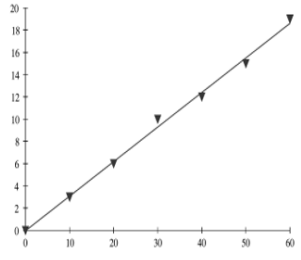

Analyte Concentration

Figure 21: Schematic diagram illustrating the simplicity of colorimetric detection. Essentially, colorimetric assays produce a colour change that can be directly related to the quantity of analyte present in a sample.

The primary purpose of this thesis research is to develop a portable sensor, for trace arsenic determination, which can be used in developing nations such as Bangladesh. To this end, I have outlined what I think are five essential characteristics of such a device: sensitivity and selectivity, speed and reproducibility, portability and robustness, health and environmental safety, and affordability and ease of use. I have also presented a critical review of various potential methods of arsenic detection and their performance with respect these criteria. From the results of this extensive analysis, I decided to choose colorimetric detection as my method of choice for the development of my arsenic sensor. In comparison to other detection methods, colorimetric assays require less specialized apparatuses or expertise to perform and analyse. I believe that this simplicity renders colorimetry most suitable for portable arsenic detection in resource-limited communities. Figure 21 illustrates a conceptual schematic of colorimetric detection.

There are two classical reactions for colorimetric arsenic detection: the Gutzeit method and molybdenum blue chemistry. As described in the literature review, colorimetric detection of arsenic by the Gutzeit method has been employed extensively in existing arsenic field test kits. In addition to the inherent health risks posed by the toxic nature of the reaction, which involves mercuric bromide and the evolution of toxic arsine gas, this method is also unreliable for trace arsenic monitoring and well-labelling. The objective of my experimental research is to improve upon the shortcomings of the Gutzeit method, for portable arsenic detection. With health risks and low reliability of existing field test kits as my two key benchmarks for improvement, I decided to base my colorimetric arsenic sensor on molybdenum blue chemistry. Although there are several colorimetric arsenic assays in the literature that are based on 
molybdenum blue chemistry, I have attempted to improve upon and miniaturize the particular assay developed by Morita and Kaneko (Figure 6-a).

Molybdenum blue chemistry for arsenic detection is based on the reaction between arsenic and molybdenum to give a coloured heteropolyacid ion product. The lack of highly poisonous reagents or arsine gas generation in the molybdenum blue assay, renders it a much safer alternative for routine arsenic detection in comparison to the Gutzeit method. However, there are three main challenges associated with this chemistry: 1) The low molar absorptivity of the produced arsenomolybdate species often calls for spectrophotometric detection during trace arsenic determinations; 2) The reaction will only proceed with arsenic in the pentavalent state, and so any trivalent arsenic species will not be detected; and 3) Any phosphates and silicates present in solution will react with molybdenum and interfere with the reaction of interest. I note that issues of speciation requirements and chemical interference are not challenges unique to arsenic detection, they are rather general challenges of sample work-up and preparation for chemical analysis and there is much research in the literature that has been devoted to solving these problems. As a result, I will not specifically focus on these issues during the development of my assay. Instead, I have chosen to work with the Morita and Kaneko assay because it yields an arsenomolybdate product which can be visually detected without the use of spectrophotometry.

a.

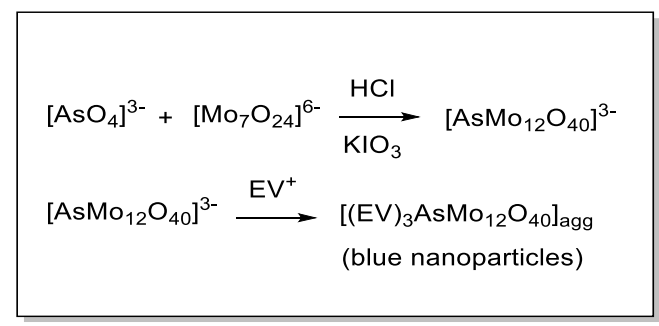

b.

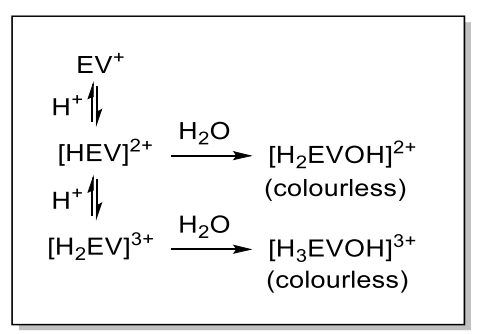

Figure 22: Chemical reaction schemes of the colorimetric arsenic assay to be employed in my portable arsenic sensor (Morita \& Kaneko 2006a). a. Under oxidizing conditions, arsenate ions in solution react with molybdate ions to form an arsenomolybdate heteropoly anion, which then forms a coloured, ionic salt with ethyl violet (EV) dye in solution. b. Under acidic conditions, excess EV ions in solution react with water to form colourless catechol species. The net result of the two sets of reactions is a colorimetric change that is a function of arsenic concentration in solution.

The chemistry presented by Morita and Kaneko takes the classical molybdenum blue reaction one step further to generate a stable coloured product (Figure 22). Here, pentavalent arsenates in solution are reacted with ammonium heptamolybdate in the presence of potassium iodate and strongly acidic conditions. The resultant arsenomolybdate heteropolyacid is reacted with the cationic dye, ethyl violet, to form coloured nanoparticles. Under acidic conditions, any unreacted dye is converted to a colourless product at equilibrium. The resultant nanoparticles have a high molar absorptivity which allows their colour to be 
easily detected by the naked eye or camera. This property renders this method well suited for the field. Furthermore, the simplicity of the assay, which is essentially just a mixing of reagents with water, renders the Morita and Kaneko reaction quite suitable for portable implementation.

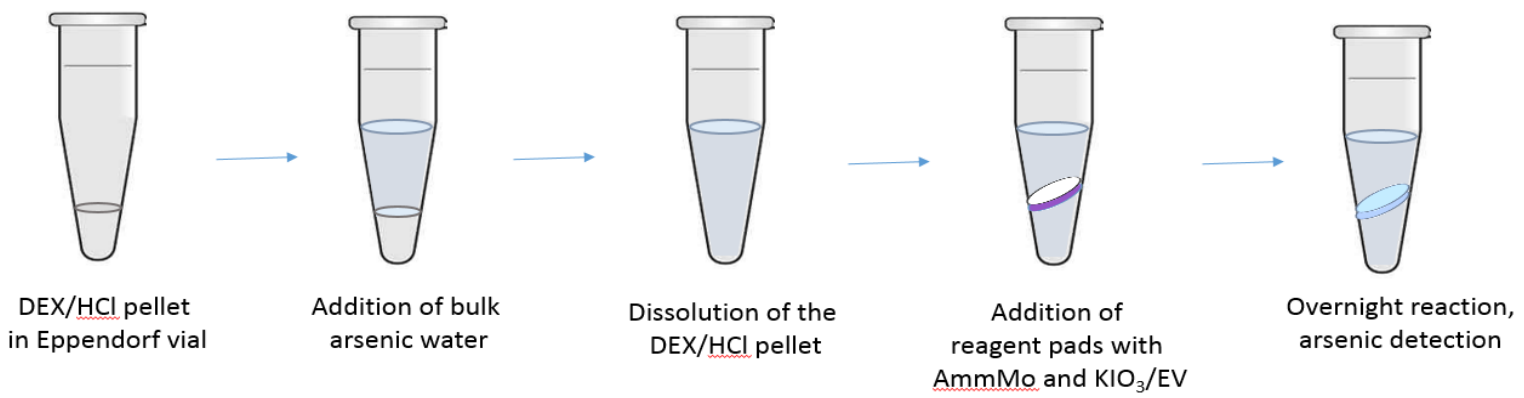

Figure 23: Schematic diagram of my final portable assay for arsenic detection, based on the Morita and Kaneko reaction. DEX/HCl pellets are prepared in Eppendorf vials and re-dissolved in arsenic water; arsenic detection then proceeds by overnight reaction following the addition of AmmMo and $\mathrm{KIO}_{3} / \mathrm{EV}$ reagent pads.

The main focus of my research is to engineer a simplified protocol of the Morita and Kaneko assay such that it can be easily and reproducibly used on the field, in a region such as Bangladesh, by a person of little specialised training. Addressing my criteria for an ideal sensor, I have attempted to improve the cost, portability, and ease of use of the original assay. To achieve this, I have adapted the protocol to a $\mu$ PAD format in which all reagents are introduced in a pre-dried form. A schematic diagram of the modified assay is illustrated in Figure 23. In my most complete system, arsenic water is initially used for the solubilisation of an acidified dextan (DEX) pellet; this solution is then reacted with cellulose paper pads containing ammonium molybdate, potassium iodate, and ethyl violet dye. Upon reaction, the paper pads change colour, indicating the presence of arsenic. Furthermore, to improve the sensitivity and reliability of colour interpretation, I have introduced the use of a camera to image these pads and then quantify the degree of colour change. 


\subsection{MPAD AdAPTATION OF THE MORITA AND KANEKo ASSAY}

This section describes the experiments that I had conducted while initially trying to simplify the Morita and Kaneko assay. First, I verified that my adaptation of the bulk assay yields results similar to what the original authors have reported. After confirming that my approach can successfully reproduce the expected results, I then proceeded to investigate how the assay behaved when conducted with different $\mu$ PAD systems. Appendix A outlines some general methodological features that are common to all of my experiments; topics discussed include solution preparation, paper device fabrication, as well as image acquisition and analysis.

\subsubsection{Characterisation of the Bulk Assay}

\subsubsection{Methods}

The original Morita and Kaneko assay was conducted with a few modifications from the reported protocol (Morita \& Kaneko 2006a). Arsenic solution aliquots of varying concentration $(5.00 \mathrm{~mL}$; 0, 10, 25, $50,100,200,250 \mu \mathrm{g} / \mathrm{L})$ were treated with potassium iodate $\left(\mathrm{KIO}_{3}, 200 \mu \mathrm{L}\right)$, hydrochloric acid $(\mathrm{HCl}, 340$ $\mu \mathrm{L}$ ), ammonium heptamolybdate (AmmMo, $300 \mu \mathrm{L}$ ), and ethyl violet (EV, $200 \mu \mathrm{L})$. Reactions were carried out in glass vials, and reaction progress was photographically documented over time $(\mathrm{t}=30 \mathrm{~min})$.

\subsubsection{Results}

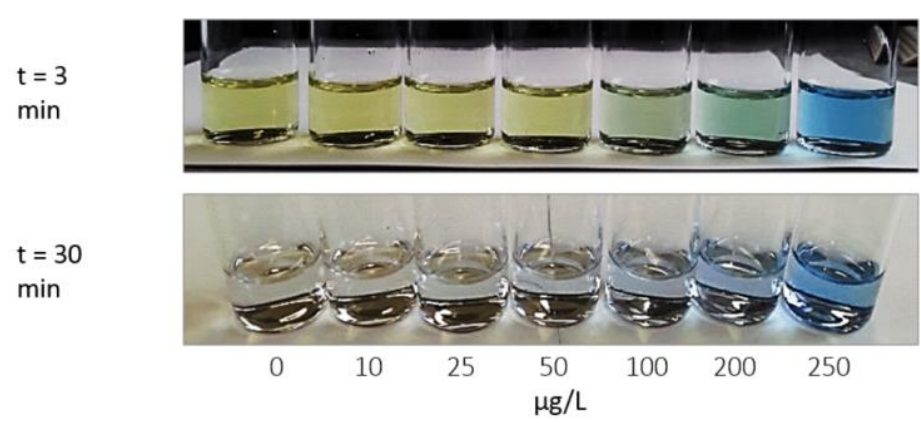

Figure 24: Results of my adaptation of the Morita and Kaneko bulk assay; color scale generated from the reaction of arsenic water $(0-250 \mu \mathrm{g} / \mathrm{L})$ with $\mathrm{KIO}_{3}$, AmmMo, $\mathrm{HCl}$, and EV, imaged with a DSLR camera. The presence of arsenic in solution can be detected by the corresponding blueness of the reaction mixture; this is true both for the early $(\mathrm{t}=3 \mathrm{~min})$ as well as equilibrium $(\mathrm{t}=30 \mathrm{~min})$ stages of the reaction.

As depicted in Figure 24, the adapted Morita and Kaneko bulk reaction yielded a colorimetric assay for arsenic. The arsenic solutions were initially clear, and remained colourless during the addition of the colourless reagents $\mathrm{KIO}_{3}$, AmmMo, and $\mathrm{HCl}$. Upon addition of purple-coloured EV, the solutions turned a pale-yellow; this yellow colour then initially proceeded to increase in saturation ( $2 \mathrm{~min}$ ) before slowly fading away completely (30 min). An increase in arsenic concentration is marked by a gradual increase in 
blueness of the reaction solution ( $3 \mathrm{~min}$ ); the assay results allow samples with arsenic levels of $100 \mu \mathrm{g} / \mathrm{L}$ or greater to be visually detected as being different from the blank. After some time $(3 \mathrm{~h})$ the blue particles generated from the reaction of arsenomolybdate with EV settle out of the bulk solution and collect at the bottom of the reaction vessel.

\subsubsection{Discussion}

Since I am using pure solutions of arsenic to approximate drinking water, I have made basic changes to the Morita and Kaneko protocol in order to eliminate some of the of the sample processing steps required for the analysis of real samples. By first performing the bulk assay with these changes, I am able to have a baseline of the colour change I should be expecting in further reactions. While providing a similar colour scale to what was initially reported by the original authors (Figure 6-a), my bulk results yielded a visual LOD of $100 \mu \mathrm{g} / \mathrm{L}$ (Morita \& Kaneko 2006a). This is somewhat higher than the $25 \mu \mathrm{g} / \mathrm{L}$ presented by Morita and Kaneko; this difference can be attributed to sample volume and imaging setup that I had used, relative to the original assay.

Furthermore, in addition to setting a colour comparison, the bulk assay gave me an understanding of certain properties of the reaction that were not described by the original authors. From conducting the bulk reactions, it is evident that the reaction between arsenic and AmmMo, and the generation of blue product with EV, proceeds quite instantly. This revealed that the length of time required to complete the assay is actually a reflection of the reaction of the excess $\mathrm{EV}$ with $\mathrm{HCl}$. In addition, observing the reaction products over a much longer timescale revealed that the microparticles generated from the arsenic reaction do not always remain suspended in solution, but actually settle out with time. This observation inspires the idea for possible approaches involve the filtering or capturing of these coloured particles out of solution, for potential signal enhancement.

\subsubsection{Wicking-based $\mu$ PADs}

\subsubsection{Direct-introduction of arsenic sample solution}

\subsection{Methods}

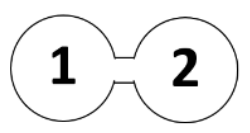

Figure 25: Schematic diagram of the wicking-based $\mu$ PADs that were used in experiments with directintroduction of the arsenic sample solution. Fabricated out of cellulose chromatography paper, devices are designed with two lobular wax channels $(r=7 \mathrm{~mm})$ that have been designated as reagent zones 1 and 2 respectively. 
As shown in Figure 25, paper devices were designed with lobular wax channels consisting of two reagent zones $(\mathrm{r}=7 \mathrm{~mm})$. The first reagent zone was prepared with a dried pre-mixed solution of KIO3, AmmMo, and $\mathrm{HCl}(1: 1.5: 1.7 ; 4.2,8.4,12.6 \mu \mathrm{L})$; the second reagent zone was prepared with dried $\mathrm{EV}(1$ $\mu \mathrm{L})$. Larger reagent volume loadings in zone 1 were accommodated through stepwise drying. Once fully dried, paper devices were cut out and laid flat on the surface of a plastic Petri dish, and the reaction was initiated by introduction arsenic solution $(25 \mu \mathrm{L})$ to the devices by direct-pipetting onto reagent zone 1 . Reaction progress was photographically documented over time.

\subsection{Results}

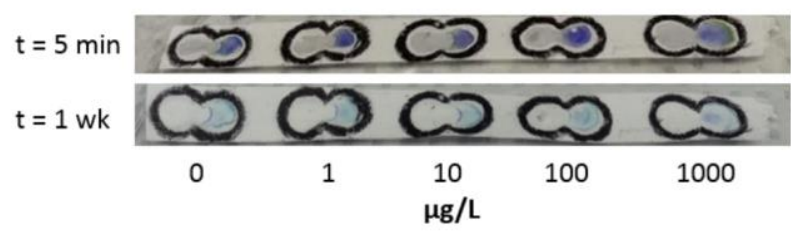

Figure 26: $\mu \mathrm{PAD}$ experiments based on horizontal wicking of arsenic water along a paper pad with predried reagents; results are shown for experiments tested with $3 x$ bulk assay reagent loadings in zone 1, imaged both immediately after reaction and after the paper pads have allowed to dry. An initial yellow front is observed in reactions conducted with $1000 \mu \mathrm{g} / \mathrm{L}$ arsenic solution.

The arsenic assay was carried out with paper pads loaded with $1 \mathrm{x}, 2 \mathrm{x}$, and $3 \mathrm{x}$ bulk assay reagent volumes in zone 1 . Even for arsenic concentrations up to $1000 \mu \mathrm{g} / \mathrm{L}$, experiments with $1 \mathrm{x}$ and $2 \mathrm{x}$ reagent loadings appear to yield the same results as those with the blank solution; that is, no appreciable reaction can be observed. However, in experiments with 3x reagent loading, $1000 \mu \mathrm{g} / \mathrm{L}$ arsenic solutions exhibit a yellow leading front while wicking across the purple-coloured EV zone of the paper pad (Figure 26). This variation in colour was only detected at early times of the reaction progress, and is not evident in the devices once they are allowed to dry. Similar behaviour was observed for three replicates of the experiment. In all experimental trials $(1 \mathrm{x}, 2 \mathrm{x}$ and $3 \mathrm{x}$ reagent loadings), the purple colour of the EV zone gradually fades to a faint blue over the course of a week's time.

\subsection{Discussion}

These experiments represent my first attempt to engineer a $\mu \mathrm{PAD}$ platform for performing the Morita and Kaneko assay. This was designed to be a single-step process in which all reagents, other than arsenic, are pre-dried onto the paper pad. Commonly $\mu$ PADs designed for environmental analysis are similar in design to that pictured in Figure 9. Typically, these devices are based on biological detection, and consist of branched channel geometries with all assay reagents (enzymes) loaded directly within the detection zone; to run the reaction, the devices are dipped into the sample, and water wicks through the 
channel to the reaction zone (Hossain et al. 2012). A fundamental difference in my initial system is that it consists of two separate reagent zones; so in order for the reaction to proceed, the sample water must transport reagents from one location on the pad to another.

The availability of pre-dried reagents to participate in the reaction depends on the fraction that leaves the paper substrate and enters the bulk solution. In particular, the reagent in zone 1 must dissolve off of the paper, enter the bulk solution, and then be carried by the fluid flow to zone 2. In my devices, when initial paper pads with $1 \mathrm{x}$ reagent loadings in zone 1 did not exhibit any reaction, increased reagent loadings were tested in an attempt to increase the concentration of reagents entering the bulk solution. The observations for the reactions with $3 \mathrm{x}$ reagent loadings in zone 1 are indicative that the reagents were in fact entering the bulk solution traveling to zone 2. However, the resultant colour change, the development of a yellowish bulk solution leading front for the $1000 \mu \mathrm{g} / \mathrm{L}$ arsenic solutions, is different from that observed for the bulk reaction. Nevertheless, the reproducibility of this effect, in combination with the fact that a visual difference was only observed in higher concentration arsenic solutions, encouraged me to try further experiments with $\mu \mathrm{PAD}$ sensors. In particular, two improvements are required in further systems: 1 . elimination of the need for specialised equipment to measure and introduce arsenic solution to the reaction; and 2. improving the sensitivity of the sensor by two orders of magnitude, such that it approaches the WHO safe drinking water limit of $10 \mu \mathrm{g} / \mathrm{L}$.

\subsubsection{Indirect-introduction of arsenic sample solution}

\subsection{Methods}

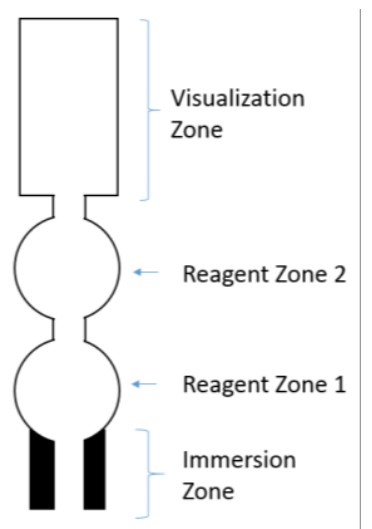

Figure 27: Sample schematic diagram of a wicking-based $\mu$ PAD that was used in experiments with indirect-introduction of the arsenic sample solution. Fabricated out of cellulose chromatography paper, devices are designed with a sample immersion zone; two-lobed wax channels $(r=7 \mathrm{~mm})$, designated as reagent zones 1 and 2 respectively; and a product visualisation zone. Arsenic solution is introduced to the reaction by dipping the immersion zone into a reservoir of bulk solution. 
As shown in Figure 27, devices were designed with lobular wax channels consisting of an arsenic introduction zone, reagent zones, and a product visualization zone. Reagent zone 1 was prepared with a dried pre-mixed solution of $\mathrm{KIO}_{3}, \mathrm{AmmMo}$, and $\mathrm{HCl}(1: 1.5: 1.7 ; 12.8 \mu \mathrm{L})$; zone 2 was prepared with dried EV $(1 \mu \mathrm{L})$. Larger reagent volume loadings in zone 1 were accommodated through stepwise drying. Arsenic solution was introduced to devices by dipping the sample immersion zone into a reservoir of bulk solution. Reaction progress was photographically documented over time.

Some experiments were also carried out with devices consisting of a single reagent zone. Here, only EV $(1 \mu \mathrm{L})$ is pre-dried on the paper device; and $\mathrm{KIO}_{3}$, AmmMo, and $\mathrm{HCl}$ are introduced as part of the arsenic bulk solution (1:1.5:12.8:25). Reaction progress was then photographically documented over time.

\subsection{Results}

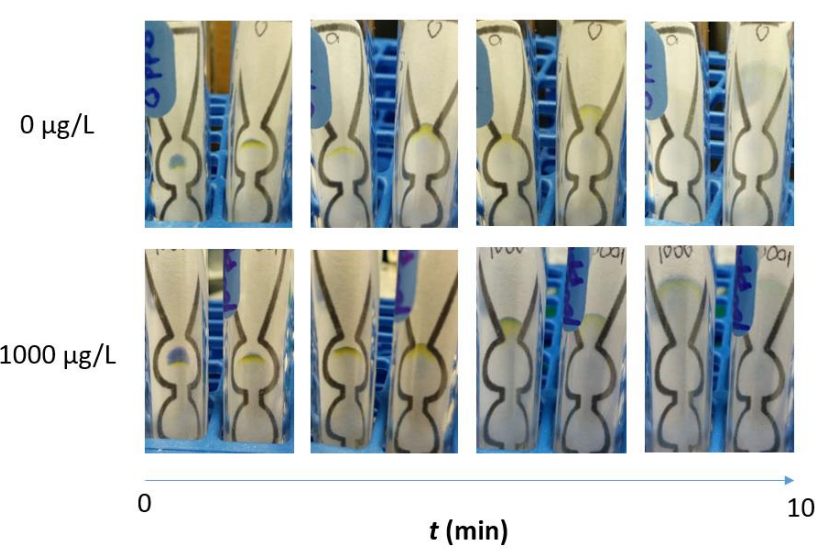

Figure 28: Time-lapse images of $\mu \mathrm{PAD}$ experiments based on vertical wicking of arsenic water along a paper pad with pre-dried reagents. Paper devices used had a wide product visualisation zone, and could not visually detect the presence of arsenic. Results are shown over a course of $10 \mathrm{~min}$, for duplicate reactions testing solutions with arsenic concentrations of 0 and $1000 \mu \mathrm{g} / \mathrm{L}$, and indicate complete reaction of the EV spot with the bulk solution. Images acquired in ambient lighting conditions.

Initial vertical wicking experiments were conducted with two-lobed paper pads with a large product visualization zone; the results from these systems could not differentiate between arsenic concentrations of 0 and $1000 \mu \mathrm{g} / \mathrm{L}$ (Figure 28). When the paper devices are immersed into arsenic solution, the bulk solution wicks up the device, through the two reagent zones, and up to the product visualisation zone. Unlike the experiments with direct arsenic sample introduction, when the fluid front wicks through zone 2, the purple EV spot first becomes yellow and eventually becomes colourless. As the front continues to move up the device, it gains a blue leading edge and yellow trailing edge; this coloured band exhibits widening with time. 
a.

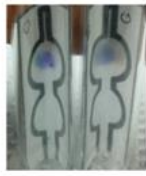

0

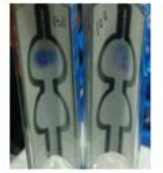

100
$\mu g / L$ b.

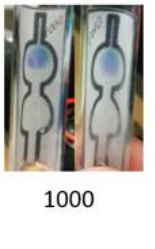

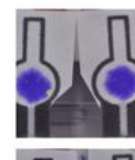
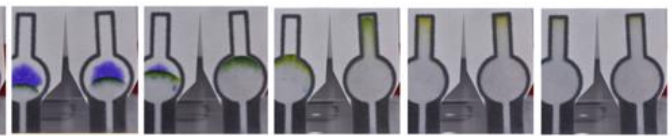

$100 \mu \mathrm{g} / \mathrm{L}$
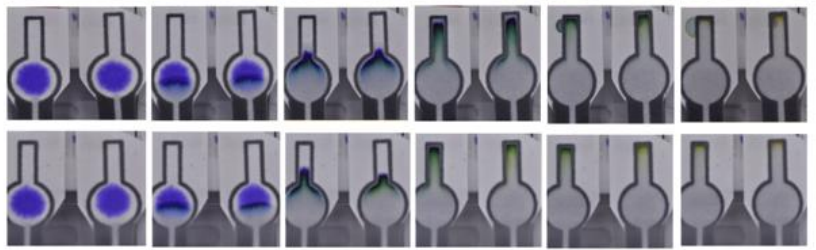

$1000 \mu \mathrm{g} / \mathrm{L}$
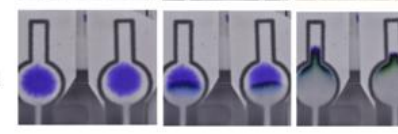

$t$ (min)

10

Figure 29: $\mu \mathrm{PAD}$ experiments based on vertical wicking of arsenic water along a paper pad with pre-dried reagents. Paper devices used had a narrow product visualisation zone; results are shown for duplicate reactions testing solutions with arsenic concentrations of 0,100 and $1000 \mu \mathrm{g} / \mathrm{L}$, over a course of $10 \mathrm{~min}$. a. Reactions with two-lobed paper pads; results are similar to those from initial horizontal wicking experiments, complete reaction of the EV spot has not occurred. Images acquired in ambient lighting conditions $b$. Reactions with single-lobed paper pads; time lapse images indicate complete reaction of the EV spot with the bulk solution. Images acquired within imaging box A.

Later vertical wicking experiments were conducted using paper pads with a narrow product visualisation zone. As shown in Figure 29-a, two-lobed devices did not exhibit complete reaction of EV with arsenic solution, this was true even for systems with increased loadings of pre-dried reagent 1. Furthermore, the narrow product visualisation zone causes the blue fluid front to be quite obscured. In contrast, complete reaction of the EV is observed in experiments with reagent 1 included in the bulk phase (Figure 29-b). With this design, the complete reaction of the EV zone is observed, and the fluid front exhibits yellow-blue banding once it reaches the product visualisation zone. In both cases, the reactions could not visually distinguish between arsenic concentrations of 0,100 , and $1000 \mu \mathrm{g} / \mathrm{L}$. Furthermore, wicking speeds through narrow-ended devices are considerably different from those through wide ended devices.

\subsection{Discussion}

The paper pads utilised in these experiments are designed in the conventional manner, with bulk solution introduction proceeding by dipping the devices into a sample reservoir. A fundamental advantage of this approach in contrast to my previous system is that there is no need for specialised equipment to measure the volume of solution to be introduced. As such, it is a simpler protocol to employ on the field.

The results from the experiments with wide-ended paper devices indicate that sufficient reagent 1 is dissolving into the bulk solution to completely react with the dried EV. However, a challenge with this geometry is that, as the generated products wick up the device, the widening product visualisation zone results in spreading of the band across a larger cross-sectional area. Narrow-ended paper devices were 
utilised in later experiments, in an attempt to concentrate the generated product to a smaller cross-sectional area. The assay protocol was the same as that for wide-ended devices, yet experiments with double-lobed narrow-ended devices did not yield complete EV reaction. This suggests that a smaller fraction of pre-dried reagent 1 entered the bulk solution. Given that both sets of devices were designed with identical reagent zone geometries, it is unclear why reagent 1 did not enter the bulk solution in the latter experiments.

In order to investigate what would happen in narrow-ended devices where reagent 1 is indeed reaching the EV zone, assays were carried out with single-lobed devices loaded only with EV and then dipped them into reagent 1-containing bulk solution. As expected, this protocol yielded complete reaction of the EV zone, and also generated more concentrated product bands. However, the system was still unable to discern the presence of arsenic. A potential explanation for these results is that the wicking rate of the paper devices does not correspond to the rate of the reaction; that is to say the arsenic solution is moving too quickly through the channel to allow visualisation of product generation.

Furthermore, the product bands generated by these experiments are indicative that the assay proceeds in a serial fashion when it is propagated by wicking. As the arsenic water-reagent 1 solution wicks across the EV spot, it generates leading and tailing ends of a product band. These ends represent different time points within the reaction; species in the leading end of the band correspond with later points in time, while species in the tailing end correspond with earlier points in time. This spatial and temporal separation of products is unfavourable for the development of a sensitive sensor. In an ideal system, all species present should undergo the reaction in a parallel fashion. That is, the arsenic solution should encounter all reagent 1 molecules at the same time, and this mixture should encounter all EV molecules at the same time.

Moreover, the rationale behind conducting the experiments via dipping is to eliminate the need for precise measurement of the volume of arsenic solution that is introduced to the device. However, my results suggest that considerable fine-tuning is required to control the rate of fluid wicking through the device. Wicking speed is a function of both paper device geometry, as well as cross-sectional area in contact with water (Fua et al. 2012). Wicking speed controls the abilities of both reagent 1 and EV to partake in the reaction, as well as the ability to visualise the generated products. In order to eliminate the dependence of my assay on wicking, I decided to engineer a $\mu \mathrm{PAD}$ system in which arsenic solution introductions by completing immersing paper pads into a sample reservoir. 


\subsubsection{Immersion-based $\mu$ PADs}

\subsubsection{Methods}

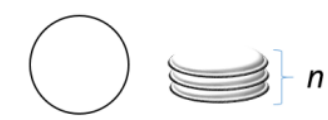

Figure 30: Schematic diagram of immersion $\mu$ PADs. Fabricated out of cellulose chromatography paper, pads are designed with a single circular reagent zone $(\mathrm{r}=5 \mathrm{~mm})$. Stacks of $n$ pads are used for the fabrication of multi-reagent devices.

As shown in Figure 30, devices were fabricated by stacking circular reagent pads. Following reagent deposition and drying into circular zones, devices are cut out to form paper reagent pads. Reactions are allowed to proceed by pipetting arsenic droplets onto the surface of a plastic Petri dish and then immersing reagent-containing pads completely in the droplets. Assays with double-pad stacked devices consisted of a reagent pad with a dried pre-mixed solution of $\mathrm{KIO}_{3}, \mathrm{AmmMo}$, and $\mathrm{HCl}(1: 1.5: 1.7 ; 12.8 \mu \mathrm{L})$ and a reagent pad with dried EV $(1 \mu \mathrm{L})$, both immersed to bulk solution droplets $(50 \mu \mathrm{L})$. Single-pad experiments were similarly carried out using pads with dried EV $(1 \mu \mathrm{L})$, immersed in droplets of arsenic bulk solution that is pre-mixed with $\mathrm{KIO}_{3}$, AmmMo, and $\mathrm{HCl}(25 \mu \mathrm{L}, 1 \mu \mathrm{L}, 1.5 \mu \mathrm{L}, 1.7 \mu \mathrm{L})$. In both cases, reaction progress was photographically documented over time.

\subsubsection{Results}

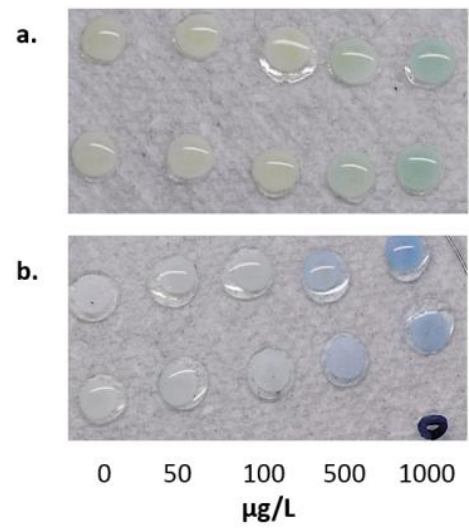

Figure 31: Results of single-pad reactions conducted, in duplicate, by immersion of EV-containing pads (r $=5 \mathrm{~mm})$ into arsenic bulk solution $(0-1000 \mu \mathrm{g} / \mathrm{L})$ containing $\mathrm{KIO}_{3}, \mathrm{AmmMo}$, and $\mathrm{HCl}$. The colour change of the immersed pads is in great agreement with that of the bulk phase experiment. a. Initial colour change exhibited by reagent pads $(t=3 \mathrm{~min})$; $b$. Equilibrium colours of reagent pads $(t=60 \mathrm{~min})$. The assay can detect the presence of arsenic with a visual LOD of $500 \mu \mathrm{g} / \mathrm{L}$. Images are acquired within imaging box A.

The presence of arsenic was not detected by double-pad experiments conducted with paper pads carrying pre-dried $\mathrm{KIO}_{3} / \mathrm{AmmMo} / \mathrm{HCl}$ (colourless) and $\mathrm{EV}$ (purple). No appreciable colour change was observed in either of the pads, for arsenic concentrations ranging from 0-1000 $\mu \mathrm{g} / \mathrm{L}$. However, single-pad experiments, conducted with paper pads carrying pre-dried EV, were capable of detecting the arsenic 
presence with a visual LOD of $500 \mu \mathrm{g} / \mathrm{L}$. Upon immersion into the colourless, $\mathrm{KIO}_{3} / \mathrm{AmmMo} / \mathrm{HCl}$ containing bulk solution, the originally purple-coloured EV pad initially turns yellow ( $\mathrm{t}=3 \mathrm{~min}$ ), with solutions containing arsenic giving the pads a green tinge. As the reaction proceeds, the pads lose their yellowness completely $(\mathrm{t}=60 \mathrm{~min})$ and the resultant outcome is a sequence of concentration-dependent blue-coloured pads (Figure 31). The intensity of the evolved blue colour corresponds to the concentration of arsenic in solution.

a.
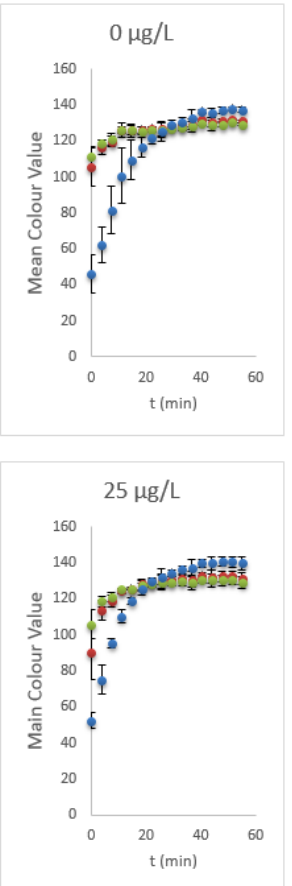
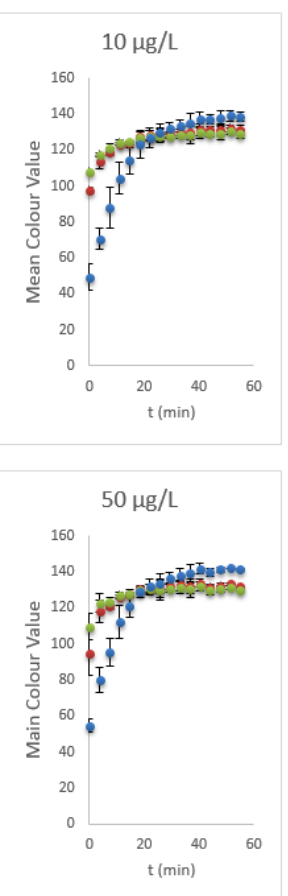

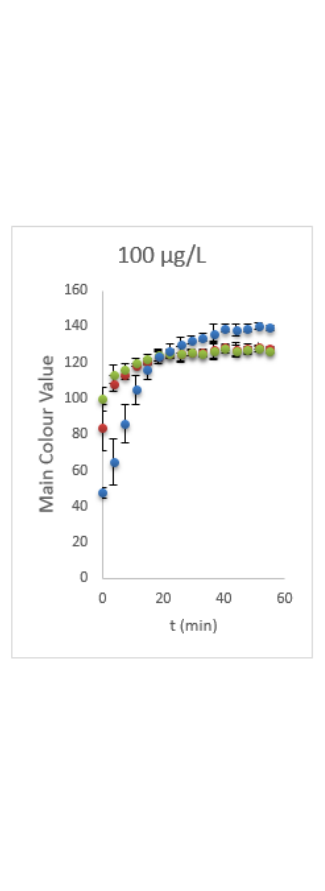

b. $20 \quad$ Equilibrium Response ( $t=50 \mathrm{~min})$

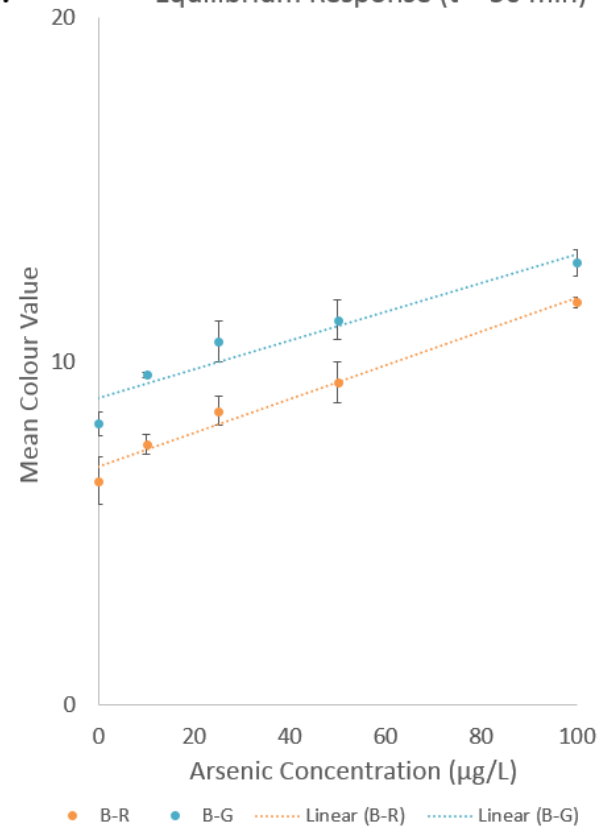

Figure 32: RGB colour analysis of results from single-pad immersion experiments $(n=3)$, conducted by immersing EV-pads in arsenic bulk solution containing $\mathrm{KIO}_{3}, \mathrm{AmmMo}$, and $\mathrm{HCl}$. a. Plots of RGB mean colour-response as a function of time, generated from time-lapse images of assays testing arsenic solutions of $0-100 \mu \mathrm{g} / \mathrm{L}$. b. Plot of the derived colour responses B-R and B-G, at equilibrium, as a function of arsenic concentration $(0-100 \mu \mathrm{g} / \mathrm{L})$. Error bars represent the observed spread in replicate responses.

Table 4: Table of standard regression parameters of linear fits applied to the B-R and B-G equilibrium responses obtained for single-pad immersion experiments. The linear equation of best fit, correlation coefficient $(\mathrm{R})$, standard deviation of the regression $\left(\mathrm{s}_{\mathrm{y} / \mathrm{x}}\right)$, precision $\left(\mathrm{s}_{\mathrm{y} / \mathrm{x}} / \mathrm{slope}\right)$, and limit of detection ( $3 \mathrm{~s}_{\mathrm{y} / \mathrm{x}} / \mathrm{slope}$; calculated based on a $1 \%$ confidence limit) of the two responses are tabulated.

\section{\begin{tabular}{|l|l|}
\hline Linear (B-R) & Linear (B-G) \\
\hline
\end{tabular}}

\begin{tabular}{|l|l|l|}
\hline \multicolumn{2}{|l|}{ Regression Parameters } & \multicolumn{2}{l|}{} \\
\hline Regression Equation & $\mathrm{B}-\mathrm{R}=0.0489[\mathrm{As}]+6.9484$ & $\mathrm{~B}-\mathrm{G}=0.0419[\mathrm{As}]+8.9441$ \\
\hline Correlation Coefficient $(\mathrm{R})$ & 0.9653 & 0.9316 \\
\hline Standard Deviation $\left(\mathrm{s}_{\mathrm{y} / \mathrm{x}}\right)$ & 1.8854 & 2.3322 \\
\hline
\end{tabular}




\begin{tabular}{|l|l|l|}
\hline \multicolumn{2}{|l|}{ Analytical Parameters } \\
\hline Precision $\left(\mathrm{s}_{\mathrm{y} / \mathrm{x}} / \mathrm{slope}\right)$ & $\pm 4 \mu \mathrm{g} / \mathrm{L}$ & $\pm 6 \mu \mathrm{g} / \mathrm{L}$ \\
\hline LOD $^{*}\left(3 \mathrm{~s}_{\mathrm{y} / \mathrm{x}} / \mathrm{slope}\right)$ & $12 \mu \mathrm{g} / \mathrm{L}$ & $17 \mu \mathrm{g} / \mathrm{L}$ \\
\hline
\end{tabular}

Time-lapse images ( 1 per every $6 \mathrm{~min})$ of triplicate single-pad experiments $(0-100 \mu \mathrm{g} / \mathrm{L})$ were analysed using the Histogram function in ImageJ and average red, green, and blue (RGB) colour channel values were plotted as a function of time (Figure 32-a). The three colour channels exhibit increasing values as a function of time; moreover, this trend is non-linear and the curves plateau after approximately 50 minutes. The profile of the blue colour channel exhibits similar values for all concentrations plotted, while profiles of the red and green channels appear to be concentration-dependent. Using the blue curve as a normalisation for ambient conditions, I have defined the additional colour functions, B-R and B-G, as the difference between the red and green channels and blue channel respectively. Calibration plots as a function of arsenic concentration were then plotted for equilibrium images, using these defined parameters (Figure 32-b). The calibration curves were fit to a linear regression, the parameters of which are given in Table 4. The plot of B-R is the most sensitive to the presence of arsenic, this calibration has a precision of $\pm 4 \mu \mathrm{g} / \mathrm{L}$ and LOD of $12 \mu \mathrm{g} / \mathrm{L}$; the calibration based on the B-G plot yields precision and LOD values of $\pm 6 \mu \mathrm{g} / \mathrm{L}$ and $17 \mu \mathrm{g} / \mathrm{L}$ respectively.

\subsubsection{Discussion}

Engineering of the $\mu \mathrm{PAD}$ assay into a paper pad protocol greatly improved the performance of the system with regards to arsenic detection. As expected, conducting the reaction in a parallel manner increases the ease of analysing the generation of the coloured product. Furthermore, through comparison of Figures 31 and 32, it becomes quite evident that digital quantification can greatly improve sensitivity of the paperpad assay (up to 50-fold). With digital colour analysis, the single-pad reaction can detect arsenic concentrations that are very close to the WHO defined MCL of $10 \mu \mathrm{g} / \mathrm{L}$, using the B-R parameter. These preliminary results indicate that arsenic determination based on B-R exhibits far superior sensitivity to the arsenic field test kits that are currently on the markets

The purpose of colour modeling is to accurately deconstruct into variables and represent a particular colour space (Ibraheem et al. 2012). In RGB colour theory, colour values range from 0-255, with (0, 0, 0) corresponding to black and $(255,255,255)$ corresponding to white. In addition to RGB, histogram values of the assay results were also plotted using two other existing colour models, HSV and YCbCr. The parameters B-R and B-G were defined because the existing colour models investigated did not produce an adequately usable calibration for the arsenic response. The observation that the parameters B-R and B-G increase with concentration indicate that pads from higher concentrations have a more dominant colour 
evolution. Notably, the LOD from the B-R curve $(12 \mu \mathrm{g} / \mathrm{L})$ approaches the LOD reported by the original authors based on UV-Vis analysis of the reaction products from the bulk system $(4 \mu \mathrm{g} / \mathrm{L})$. I use this sensitivity as a benchmark to evaluate any further improvements of the paper-based assay.

Since the arsenic detection assay was able to proceed in single-pad experiments, but not in doublepad experiments, this indicates that components of the double-pad are insufficiently released from the paper to undergo complete reaction. Some explanations for this behaviour may be that the assay reagents become inactivated by undergoing reaction either with one another or with the paper substrate; or that particular reagents are inherently more tightly bound to the paper substrate than others; or that intermediate reaction products adhere to the paper substrate as they are formed, effectively blocking further reactions from proceeding. However, despite its superior sensitivity, the described single-pad assay is not suitable for use as a portable sensor; liquid reagents are difficult to package, transport, and administer on the field. For ease of use in arsenic-affected regions, it is necessary to simplify the assay and remove the need for liquid reagents. The following section describes the steps that I have taken to tackle this challenge. 


\subsection{ELIMINATION OF LIQUID REAGENTS FROM THE $\mu$ PAD SENSOR}

This section describes the experiments that I conducted in order to transform my immersion-based $\mu \mathrm{PAD}$ into a liquid-free portable sensor for arsenic detection. The first step that I took toward simplification was reduction of the number of reagents required for the assay. To do so, I needed to learn more about the chemistry of the reagents involved; I conducted experiments to determine which sets of reagents were compatible with one another and could thus be premixed without affecting the arsenic detection reaction. Once I defined my system of regents, I then proceeded to methodologically introduce each to the assay in a liquid-free form. Contrary to my first sequence of experiments, which were explorative in nature and primarily served to understand various methods of $\mu \mathrm{PAD}$ development, these latter experiments systematically approach the challenge of attempting to fine-tune my $\mu \mathrm{PAD}$ assay into a fully portable system.

\subsubsection{Determination of Reagent Incompatibilities}

\subsubsection{Methods}

The four fundamental regents of the Morita and Kaneko assay, $\mathrm{KIO}_{3}(20 \mu \mathrm{L})$, AmmMo $(30 \mu \mathrm{L})$, $\mathrm{HCl}(34 \mu \mathrm{L})$, and $\mathrm{EV}(20 \mu \mathrm{L})$ were pre-mixed in various combinations, and allowed to sit overnight. Arsenic solution aliquots of varying concentration $(0,1000 \mu \mathrm{g} / \mathrm{L} ; 500 \mu \mathrm{L})$ were treated with each series of unaffected pre-mixed reagent combinations. Reaction progress was compared against that of my original bulk assay $(\mathrm{t}=30 \mathrm{~min})$. Reactions were carried out in Eppendorf vials.

\subsubsection{Results}

Table 5: Observations of pre-mixed reagent solutions of $\mathrm{KIO}_{3}, \mathrm{AmmMo}, \mathrm{HCl}$ and $\mathrm{EV}(20.30,34,20 \mu \mathrm{L})$ left to sit overnight. The reagent combinations that exhibited no change (marked with asterisk $(*)$ ) were then reacted with arsenic solution $(500 \mu \mathrm{L})$; when reacted with arsenic, all four of these combinations exhibited similar behaviour as the original bulk experiment $(* *)$.

\begin{tabular}{|r|c|c|c|c|c|}
\hline \multicolumn{1}{c|}{$\#$} & Reagent 1 & Reagent 2 & Reagent 3 & Reagent 4 & Observations \\
\hline$* * 1$ & $\mathrm{KIO}_{3}$ & $\mathrm{AmmMo}$ & $\mathrm{HCl}$ & EV & No change \\
\hline$* 2$ & $\mathrm{KIO}_{3} / \mathrm{AmmMo}$ & $\mathrm{HCl}$ & $\mathrm{EV}$ & - & No change \\
\hline 3 & $\mathrm{KIO}_{3} / \mathrm{HCl}$ & $\mathrm{AmmMo}$ & $\mathrm{EV}$ & - & R1 turns yellow (overnight) \\
\hline$* 4$ & $\mathrm{KIO}_{3} / \mathrm{EV}$ & $\mathrm{AmmMo}$ & $\mathrm{HCl}$ & - & No change \\
\hline$* 5$ & $\mathrm{AmmMo} / \mathrm{HCl}$ & $\mathrm{KIO}_{3}$ & $\mathrm{EV}$ & - & No change \\
\hline 6 & $\mathrm{AmmMo} / \mathrm{EV}$ & $\mathrm{KIO}_{3}$ & $\mathrm{HCl}$ & - & R1 precipitates (overnight) \\
\hline 7 & $\mathrm{HCl} / \mathrm{EV}$ & $\mathrm{KIO}_{3}$ & $\mathrm{AmmMo}$ & - & R1 turns yellow (immediate) \\
\hline 8 & $\mathrm{KIO}_{3} / \mathrm{AmmMo}$ & $\mathrm{HCl} / \mathrm{EV}$ & - & - & R2 turns yellow (immediate) \\
\hline
\end{tabular}




\begin{tabular}{|r|c|c|c|c|c|}
\hline 9 & $\mathrm{KIO}_{3} / \mathrm{HCl}$ & $\mathrm{AmmMo} / \mathrm{EV}$ & - & - & $\begin{array}{c}\text { R1 turns yellow (overnight) } \\
\text { R2 precipitates (overnight) }\end{array}$ \\
\hline$* 10$ & $\mathrm{KIO}_{3} / \mathrm{EV}$ & $\mathrm{AmmMo} / \mathrm{HCl}$ & - & - & No change \\
\hline 11 & $\mathrm{KIO}_{3} / \mathrm{AmmMo} / \mathrm{HCl}$ & $\mathrm{EV}$ & - & - & R1 turns yellows (overnight) \\
\hline 12 & $\mathrm{KIO}_{3} / \mathrm{AmmMo} / \mathrm{EV}$ & $\mathrm{HCl}$ & - & - & $\mathrm{R} 1$ precipitates (overnight) \\
\hline 13 & $\mathrm{KIO}_{3} / \mathrm{HCl} / \mathrm{EV}$ & $\mathrm{AmmMo}$ & - & - & R1 turns yellow (immediate) \\
\hline 14 & $\mathrm{AmmMo} / \mathrm{HCl} / \mathrm{EV}$ & $\mathrm{KIO}_{3}$ & - & - & R1 precipitates (overnight) \\
\hline
\end{tabular}

Table 5 lists the 14 different reagent permutations which were tested for compatibility with one another. The following reagent incompatibilities were revealed through systematic testing of the different combinations: $\mathrm{HCl} / \mathrm{EV}, \mathrm{KIO}_{3} / \mathrm{HCl}$, and EV/AmmMo. The reagent mixtures which exhibited no change (\#2, $\# 4$, \#5, \#10), were then successfully reacted with arsenic solution to yield comparable results as my original bulk assay (\#1).

\subsubsection{Discussion}

The purpose of this experiment is to systematically determine the best way to pre-group the four reagents $\mathrm{KIO}_{3}, \mathrm{AmmMo}, \mathrm{HCl}$, and $\mathrm{EV}$ for use in the arsenic detection assay. In previous experiments, the decision to group assay reagents as $\mathrm{KIO}_{3} / \mathrm{AmmMo} / \mathrm{HCl}$ and $\mathrm{EV}$ was arbitrarily based on the grouping of colourless and coloured chemicals. Through compatibility testing, it becomes evident that this was an inappropriate choice as $\mathrm{KIO}_{3}$ and $\mathrm{HCl}$ cannot be pre-mixed with one another. This observation may serve to explain why experiments with these reagents grouped together did not produce the expected reaction results. The compatibility tests have revealed four reagent mixtures that can be stably stored; when reacted, these reagent combinations can produce arsenic colour changes similar to the bulk reaction. Therefore, when these combinations are employed in a $\mu \mathrm{PAD}$ setup, any lack of reaction can be attributed to the engineering parameters of the paper assay.

As the overarching goal of this work is simplicity of the total assay, the ideal reagent combination to test further is $\# 10, \mathrm{KIO}_{3} / \mathrm{EV}$ and $\mathrm{AmmMo} / \mathrm{HCl}$, as it is only a 2-component system. This is the first reagent system I work with in further experiments where I continue to optimise my immersion $\mu \mathrm{PAD}$ into a liquid-free portable arsenic assay.

\subsubsection{Liquid-free Reagent Delivery}

\subsubsection{Methods}

Reactions are allowed to proceed by pipetting arsenic droplets onto the surface of a plastic Petri dish and then immersing reagent-containing pads completely in the droplets. Paper pads are identical to those from initial immersion experiments (Figure 30). $\mathrm{KIO}_{3}(3 \mu \mathrm{L}), \mathrm{AmmMo}(4.5 \mu \mathrm{L}), \mathrm{HCl}(5.1 \mu \mathrm{L}$ ), and 
EV $(3 \mu \mathrm{L})$ were incorporated in the assay either by stacking pads of different dried reagents one atop the other, or as liquid components of the bulk arsenic droplet ( $25 \mu \mathrm{L} / \mathrm{pad})$. Reactions were allowed to proceed overnight; after reaction, paper pads are visually analysed following rehydration.

\subsubsection{Results}

a.

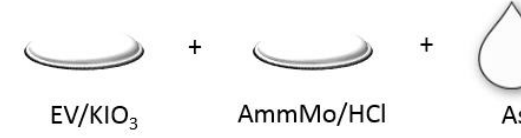

b.

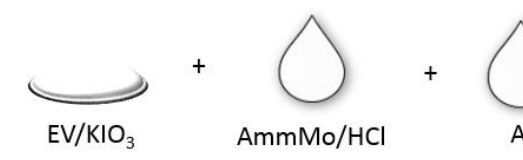

c.

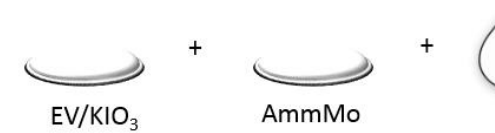

d.

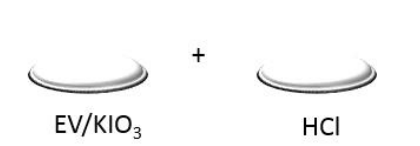

e.

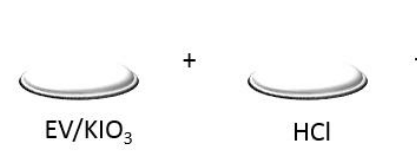

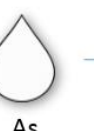

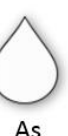

As

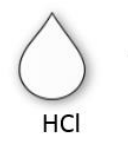

$+$
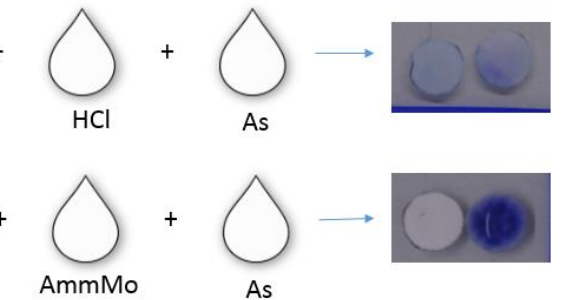

As

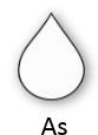

As
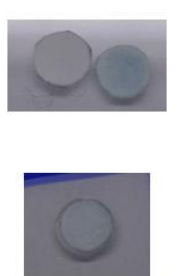

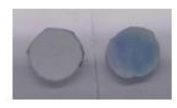

(some reaction)

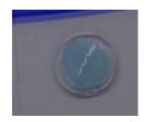

(reaction)

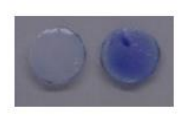

(reaction)
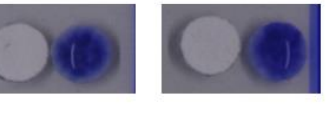

(no reaction)

(reaction) $\mathrm{gg} / \mathrm{L}$

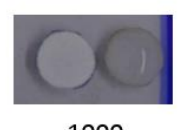

1000

Figure 33: Schematic diagram of the various $\mu \mathrm{PAD}$ assay permutations tested, along with assay results; pad and droplet shaped symbols indicate reagent delivery either as a paper pad or in liquid-form. a. Doublepad assay of $\mathrm{EV} / \mathrm{KIO}_{3}$ and $\mathrm{AmmMo} / \mathrm{HCl}$ with arsenic, some reaction observed. b. Single-pad assay of $\mathrm{EV} / \mathrm{KIO}_{3}$ with $\mathrm{AmmMo} / \mathrm{HCl}$ and arsenic; complete reaction observed. c. Double-pad assay of $\mathrm{EV} / \mathrm{KIO}_{3}$ and AmmMo, with $\mathrm{HCl}$ and arsenic; complete reaction observed. d. Double-pad assay of $\mathrm{EV} / \mathrm{KIO}_{3}$ and $\mathrm{HCl}$, with AmmMo and arsenic; no reaction observed. e. Double-pad assay of $\mathrm{EV} / \mathrm{KIO}_{3}$ and $\mathrm{HCl}$, with arsenic; complete reaction observed. Double-pad assays imaged after opening apart paper stacks; all images acquired within image box A.

The systematically tested $\mu \mathrm{PAD}$ and reagent combinations are given in Figure 33. The double-pad assay of combination \#10, with both $\mathrm{EV} / \mathrm{KIO}_{3}$ and $\mathrm{AmmMo} / \mathrm{HCl}$ on paper pads exhibited only partial reaction. Successive experiments, involving a single-variable changes to the delivery of $\mathrm{AmmMo} / \mathrm{HCl}$, revealed that the assay proceeds to completion when $\mathrm{AmmMo} / \mathrm{HCl}$ are both in solution and when AmmMo is on paper and $\mathrm{HCl}$ is in solution, but not when $\mathrm{HCl}$ is on paper and $\mathrm{AmmMo}$ is in solution. It was also determined that, in the complete absence of $\mathrm{AmmMo}, \mathrm{HCl}$ on paper can indeed react with $\mathrm{EV} / \mathrm{KIO}_{3}$ on paper. Experimental setups maintaining $\mathrm{AmmMo} / \mathrm{HCl}$ on paper with $\mathrm{EV} / \mathrm{KIO}_{3}$ in solution were not explored due to imaging restrictions posed by introducing the deeply purple EV in liquid form. 


\subsubsection{Discussion}

The purpose of these experiments is to determine the behaviour of the reagent groupings from combination \#10 $\left(\mathrm{EV} / \mathrm{KIO}_{3}, \mathrm{AmmMo} / \mathrm{HCl}\right)$ when used in $\mu \mathrm{PAD}$ arsenic assays. The results of the $\mu \mathrm{PAD}$ tests reveal that combination $\# 4\left(\mathrm{EV} / \mathrm{KIO}_{3}, \mathrm{AmmMo}, \mathrm{HCl}\right)$ may be a better reagent sequence than combination \#10 for this application. The results indicate that the successfulness of a $\mu$ PAD assay is very dependent on the timing of reagent delivery. Reagents delivered via bulk solution are more readily available to undergo arsenic reaction than those delivered via pre-drying onto the paper substrate. Specifically, from the results of my assay, it can be inferred that is important for $\mathrm{HCl}$ to be an active reagent in solution, prior to the introduction of AmmMo. This observation may be related to the fact that, in addition to arsenic reaction, AmmMo can also undergo precipitate formation with EV. Another potential explanation may be that AmmMo binds to and blocks the surface of paper substrate. If the reaction of $\mathrm{AmmMo} / \mathrm{EV}$ or $\mathrm{AmmMo} /$ paper proceeds faster than either the reaction of AmmMo/As, AmmMo-As/EV or $\mathrm{HCl} / \mathrm{EV}$, then this can result in interference of the arsenic detection assay.

The issue of reagent timing can be addressed by dividing the experimental protocol into a two-step process in which $\mathrm{HCl}$ is delivered first, prior to the delivery of AmmMo. In the current setup, the arsenic detection assay, involving $\mathrm{EV} / \mathrm{KIO}_{3}$ on paper, proceeds most successfully only when $\mathrm{AmmMo} / \mathrm{HCl}$ are both delivered via the bulk matrix or when AmmMo is delivered on paper and $\mathrm{HCl}$ is delivered as a

component of the bulk. However, additional modifications are yet required for the complete elimination of liquids and precise reagent measuring from the assay protocol. A triple-pad system is viewed as unfavourable as it is likely to involve high product loss and signal dilution due to the additional paper layers. An alternate means of designing a liquid-free assay is through the consideration of alternate solid substrates for reagent delivery.

\subsubsection{Reagent Introduction via DEX Pellets}

\subsubsection{Methods}

Dextran solutions consisted of the DEX-rich portion of an aqueous two-phase solution (ATPS) system of DEX (MW 500k, Pharmacosmos, Holbaek, Denmark) and polyethylene glycol (PEG; MW 35k, Sigma-Aldrich, St. Louis, Mo, USA). A PEG-DEX ATPS system (20\% w/v, 26.5\% w/v) is prepared using DI water, and left undisturbed to phase-separate (2 days) (Atefi et al. 2014). The high-density DEX rich phase (bottom) is then extracted using a plastic syringe.

My simplified bulk assay of the Morita and Kaneko reaction was conducted with the inclusion of $\mathrm{DEX}$ as an additional reagent $(340 \mu \mathrm{L})$. Double-pad immersion experiments consisting of $\mathrm{EV} / \mathrm{KIO}_{3}(3 \mu \mathrm{L}$, 
$3 \mu \mathrm{L})$ and AmmMo $(4.5 \mu \mathrm{L})$ were also conducted with arsenic bulk solution droplets $(50 \mu \mathrm{L})$ containing $\mathrm{HCl}(5.1 \mu \mathrm{L})$ and DEX $(5.1 \mu \mathrm{L})$.

To form HCL/DEX pellets, aliquots $(10.2,20.4,40.8,81.6 \mu \mathrm{L})$ of a 1:1 solution of DEX and $\mathrm{HCl}$ were pipetted into Eppendorf vials and allowed to dry (4 days). Upon complete drying of the pellets, arsenic bulk solution $(500 \mu \mathrm{L})$ was added to the vials; after allowing the mixture to sit for some time $(2 \mathrm{~h})$, the vials were vigorously agitated to ensure that the pellets were completely dissolved in solution. Reactions were allowed to proceed by conducting a double-pad immersion assay with EV/KIO $3(3 \mu \mathrm{L}, 3 \mu \mathrm{L})$ and AmmMo $(4.5 \mu \mathrm{L})$ pads immersed completely into the bulk solution. Paper devices are identical as those used in initial immersion experiments (Figure 30). Reactions were allowed to proceed overnight; after which, paper pads were removed from solution and imaged for analysis.

\subsubsection{Results}

a.

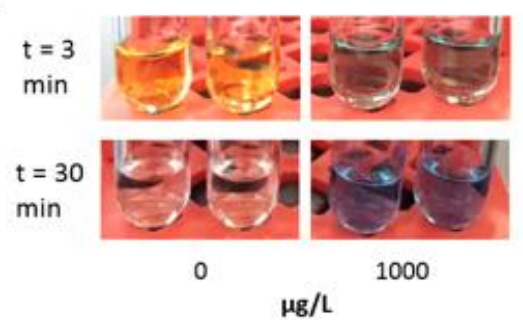

b.

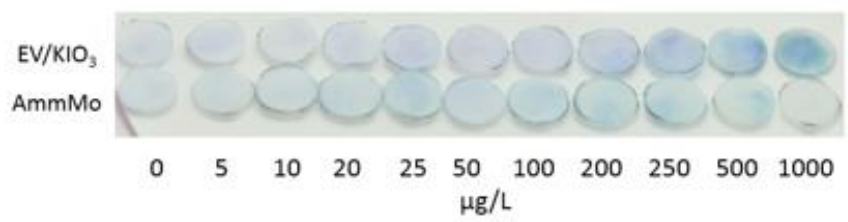

Figure 34: Results of arsenic assays involving the addition of DEX. a. Bulk experiments carried out with arsenic solutions of 0 and $1000 \mu \mathrm{g} / \mathrm{L}$ of arsenic, imaged at 3 and $30 \mathrm{~min}$; reaction conditions are the same as for Figure 24, with the additional inclusion of DEX $(340 \mu \mathrm{L})$. Images are acquired with ambient lighting conditions. b. Double-pad $\mu \mathrm{PAD}$ assays consisting of EV/ $\mathrm{KIO}_{3}$ and $\mathrm{AmmMo}$ with $8 \mathrm{x}$ loading of $\mathrm{HCl}$ introduced as a DEX-pellet. Following overnight reaction, double-pad sandwiches are removed from solution and opened apart for imaging; $\mathrm{EV} / \mathrm{KIO}_{3}$ pads exhibit an arsenic concentration dependent blue trend $(0-1000 \mu \mathrm{g} / \mathrm{L})$. Images are acquired within imaging box B.

The inclusion of DEX in the bulk arsenic assay yields more vibrantly coloured solutions than the original mixture, however the colour change exhibits the same overall trend (Figure 34-a). Furthermore, inclusion of DEX as a bulk solution component of double-pad immersion assays did not affect the results of the $\mu \mathrm{PAD}$ reaction.

Double-pad HCl/DEX pellet assays conducted with 1x, 2x, and 4x loadings of HCl/DEX did not proceed to completion. It was observed that an $\mathrm{HCl} / \mathrm{DEX}$ loading of $8 \mathrm{x}$ the bulk assay volume is required to eliminate the background signal from unreacted EV. The results of double-pad HC-DEX pellet assays conducted with arsenic solutions ranging from 0-1000 $\mu \mathrm{g} / \mathrm{L}$ are shown in Figure 34-b; this assay produces a visual LOD for arsenic of approximately $200 \mu \mathrm{g} / \mathrm{L}$. Both the $\mathrm{EV} / \mathrm{KIO}_{3}$ and $\mathrm{AmmMo}$ containing components of the paper assay develop a blue colour with the progression of the assay; the $\mathrm{EV} / \mathrm{KIO}_{3}$ pad develops a purple-blue colour and the AmmMo pad develops a green-blue colour. By visual inspection, the 
intensity of colour developed by the $\mathrm{EV} / \mathrm{KIO}_{3}$ pad appears to exhibit an increasing monotonic trend with response to arsenic concentrations, up until $500 \mu \mathrm{g} / \mathrm{L}$.

a.

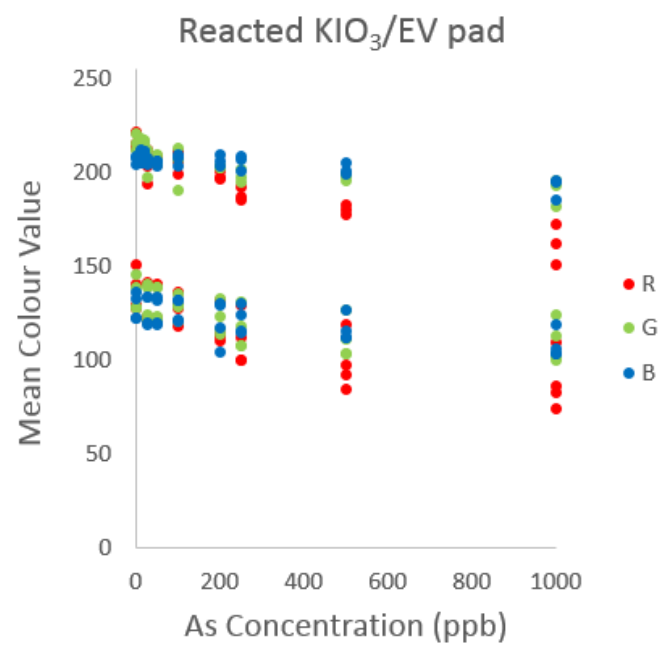

b.

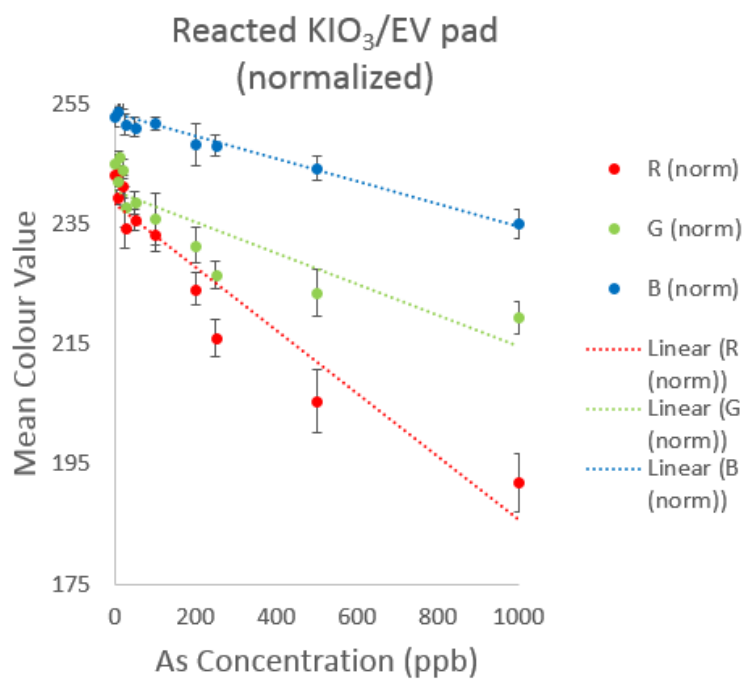

Figure 35: RGB colour analysis of results from double-pad $\mathrm{HCl} / \mathrm{DEX}$ pellet $\mu \mathrm{PAD}$ experiments (n=7); conducted by immersing $\mathrm{EV} / \mathrm{KIO}_{3}$ and AmmMo pads into arsenic bulk solution containing $8 \mathrm{x}$ loadings of re-solubilized $\mathrm{HCl} / \mathrm{DEX}$. a. Plot of RGB mean colour-response as a function of arsenic concentration, generated from reacted $\mathrm{EV} / \mathrm{KIO}_{3}$ pads; every RGB data point represents one unique experiment. b. Plot of normalised RGB colour responses of reacted $\mathrm{KIO}_{3} / \mathrm{EV}$ pads as a function of arsenic concentration; every RGB data point represents the mean response of replicate experiments. Error bars represent the observed spread in replicate responses.

Table 6: Table of standard regression parameters of linear fits applied to normalised RGB responses obtained for the reacted $\mathrm{KIO}_{3} / \mathrm{EV}$ component of double-pad HCL/DEX pellet $\mu \mathrm{PAD}$ experiments with 8x HCL loading. The linear equation of best fit, correlation coefficient $(\mathrm{R})$, standard deviation of the regression $\left(\mathrm{s}_{\mathrm{y} / \mathrm{x}}\right)$, precision $\left(\mathrm{s}_{\mathrm{y} / \mathrm{x}} / \mathrm{slope}\right)$, and limit of detection ( $3 \mathrm{~s}_{\mathrm{y} / \mathrm{x}} / \mathrm{slope}$; calculated based on a $1 \%$ confidence limit) of the three responses are tabulated.

\begin{tabular}{|l|l|l|l|}
\hline \multicolumn{2}{|l|}{ Regression Parameters } & Linear (G (norm)) & Linear (B (norm)) \\
\hline Regression Equation & $\mathrm{R}=-0.0527[\mathrm{As}]+238.4001$ & $\mathrm{G}=-0.0261[\mathrm{As}]+240.6267$ & $\mathrm{~B}=-0.01879[\mathrm{As}]+253.4207$ \\
\hline Correlation Coefficient $(\mathrm{R})$ & 0.9084 & 0.7636 & 0.9535 \\
\hline Standard Deviation $\left(\mathrm{s}_{\mathrm{y} / \mathrm{x}}\right)$ & 1.9658 & 1.7054 & 0.4875 \\
\hline Analytical Parameters & $\pm 37 \mu \mathrm{g} / \mathrm{L}$ & $\pm 65 \mu \mathrm{g} / \mathrm{L}$ & $\pm 26 \mu \mathrm{g} / \mathrm{L}$ \\
\hline Precision $\left(\mathrm{s}_{\mathrm{y} / \mathrm{x}} / \mathrm{slope}\right)$ & $112 \mu \mathrm{g} / \mathrm{L}$ & $196 \mu \mathrm{g} / \mathrm{L}$ & $78 \mu \mathrm{g} / \mathrm{L}$ \\
\hline LOD* $\left(3 \mathrm{~s}_{\mathrm{y} / \mathrm{x}} / \mathrm{slope}\right)$ & &
\end{tabular}

Mean RGB colour values of reacted $\mathrm{EV} / \mathrm{KIO}_{3}$ components ( $\mathrm{n}=7$ ) of double-pad $\mathrm{HCl} / \mathrm{DEX}$ pellet $\mu \mathrm{PAD}$ assays were determined using the Histogram function in ImageJ and plotted as a function of arsenic concentration (Figure 35-a). The resultant curves display a linear trend exhibiting decreasing RGB values 
with increasing arsenic concentration; these decreasing RGB values correspond to the increasing blueness generated by higher concentrations. However, experiments imaged during different sittings yield shifted colour values. As shown in Figure 35-b, the results from replicate experiments exhibit a much narrower spread when a linear background-correcting normalisation factor is applied to the RGB values. A linear correction factor is applied such that the background of each space surrounding the $\mathrm{KIO}_{3} / \mathrm{EV}$ pad, in each image, is defined to be white ( $\mathrm{R}=255, \mathrm{G}=255, \mathrm{~B}=255)$ (Channg \& Reid 1996).

The normalised RGB calibration curves were each fit to a linear calibration, the parameters of which are given in Table 6. The plot of normalised B values yields the most sensitive response to the presence of arsenic. Although the normalised B curve does not have a very steep response, the reproducibility of replicates lends it a precision of $\pm 26 \mu \mathrm{g} / \mathrm{L}$ and LOD of $78 \mu \mathrm{g} / \mathrm{L}$. In contrast, the plot of normalised $\mathrm{R}$ values appears to produce the steepest response as a function of arsenic concentration, but is also subject to the greatest amount of error. Moreover, the plot of normalised G values has a low correlation coefficient, indicating that it may not be changing as a direct response to arsenic.

It is to be noted that mean RGB colour values of reacted AmmMo pads, and intact double-pad stacks, do not display a monotonic trend when plotted as a function of arsenic.

\subsubsection{Discussion}

DEX has shown to be an apt alternate to paper, as a solid substrates for reagent delivery in this assay. As exemplified by the results of the bulk and initial double-pad assays, the presence of DEX does not appreciably affect the colour change yielded by arsenic detection reaction. The relative stability of this polymer is often taken advantage of in biomedical applications, where, along with PEG, it is commonly used as a component of ATPS systems. Furthermore, dehydrated DEX has recently been shown to be suitable for localized reagent delivery within these systems (Bethany et al. 2013). In my work, I have taken this idea and used DEX as a general substrate for reagent delivery. In my assays involved HCl/DEX pellets, the rehydrated pellets release reagents completely to the bulk solution; in addition, DEX does not interfere with the arsenic detection chemistry and is also transparent to light, rendering it quite suitable for use in this assay.

Furthermore, the combined application of DEX pellets, paper pads, and graduated Eppendorf vials, completely eliminates the need for precise volume measurement from the arsenic assay protocol. All assay reagents are pre-measured in their dried form, and the larger volume of the bulk solution $(500 \mu \mathrm{L})$ allows adequate measurement precision simply by using the gradations on the Eppendorf vials. In addition, the closed system of the Eppendorf vial ensures complete wetting of the paper pads throughout the entire duration of the assay; ensuring adequate reaction time and eliminating the need for rehydration prior to 
image analysis. One constraint of this setup is dilution of the $\mathrm{HCl}$ as it enters the bulk solution. An 8x reagent loading of $\mathrm{HCl}$ is required to have the reaction proceed to completion; under ambient conditions of the lab, this results in DEX pellet dry times of approximately four days. I note, however, that this limitation does not affect the commercialisation prospect of the assay, because both DEX pellets as well as paper pads have high mass-production potential.

A more pressing challenge is the fact that the presented liquid-free $\mu \mathrm{PAD}$ is unable to detect arsenic levels with adequate sensitivity to classify drinking water as safe or unsafe for consumption, even at the limit defined in developing countries of $50 \mu \mathrm{g} / \mathrm{L}$. Furthermore, this system is nearly six-fold less sensitive to arsenic than the single-pad adaptation I have described in the previous section. Since analytical sensitivity is related to the signal to noise ratio of a calibration, of the DEX-paper assay may be improved either by concentrating the detected signal or decreasing observed noise (Shrivastava \& Gupta 2011). As evidenced in Figure 34-b, the blue-coloured signal produced in the double-pad assay is split across the two paper substrates. A potential means of concentrating the observed response is by reverting the assay to a singlepad format, with $\mathrm{EV} / \mathrm{KIO}_{3}$ introduced via paper and $\mathrm{AmmMo} / \mathrm{HCl}$ both introduced via the DEX pellet. Once the AmmMo/HCl/DEX pellet dissolves in the bulk arsenic solution, this assay will be essentially the same as the double-pad experiment I used to evaluate the compatibility of DEX in $\mu$ PAD systems. Furthermore, the current calibration contains error arising from both inter-experiment variations as well as lighting inconsistencies during imaging. Errors due to imaging inconsistencies may be eliminated by mounting the camera to a fixed jig attached to image box B; in my previous experiments, this approach was very successful in taking consistent images using box A. 


\subsection{SUMmary}

The primary purpose of this thesis research is to develop a portable sensor, for trace arsenic determination, which can be used in developing nations such as Bangladesh. I have chosen colorimetric detection as my method of choice for the development of my arsenic sensor. In specific, I have worked to engineer a simplified protocol of the Morita and Kaneko assay for arsenic detection, which is based on classical molybdenum-blue chemistry. This colorimetric assay proceeds by reacting As(V) in solution with Mo(VI) under oxidizing, acidic conditions; the resultant arsenomolybdate heteropolyacid then reacts with $\mathrm{EV}$ in solution to form stable, coloured ion-aggregates. Addressing my criteria for an ideal sensor, I have attempted to improve the cost, portability, and ease of use of this original assay, via adapting it to a portable $\mu \mathrm{PAD}$. Upon confirming my ability to approximately reproduce the bulk assay results reported by the original assays, I then conducted a series of experiments to first adapt the assay to a viable $\mu$ PAD system and second to engineer it into a liquid-free $\mu \mathrm{PAD}$ system.

$\mu$ PADs were designed using wax-printed paper channels, and two approaches towards arsenic assay protocol development were explored: wicking-based $\mu$ PADs and immersion-based $\mu$ PADs. Although wicking-based $\mu \mathrm{PADs}$ have previously been reported in the literature for the environmental analysis of other trace metals, this method proved ineffective for this particular reaction. This is because all of the paper device geometries investigated generated a serial response for the arsenic detection assay, resulting in signal dilution due to spatially and temporally separated product generation. Immersion-based $\mu$ PADs avoid this issue by allowing for the reaction to proceed in a parallel manner, effectively capturing the same reaction product across the entire surface area of the paper device. Single-pad immersion experiments consisting $\mathrm{EV}$-containing paper pads immersed in $\mathrm{As} / \mathrm{KIO}_{3} / \mathrm{AmmMo} / \mathrm{HCl}$ containing bulk solution yield a comparable colometric response to the bulk assay. This assay has an arsenic LOD of $12 \mu \mathrm{g} / \mathrm{L}$, based on computerassisted calibration of reaction pad images, using the derived RGB colour parameter B-R.

In order to engineer the immersion-based assay into a liquid-free system, systematic experiments were conducted to gain insight into mechanistic aspects of the reaction. First, reagent compatibility was tested to determine the best way to pre-group the four reagents of the original assay; from these experiments, the two grouping of $\mathrm{AmmMo} / \mathrm{HCl}$ and $\mathrm{EV} / \mathrm{KIO}_{3}$ was chosen as the simplest permutation to carry forward to further testing. However, a sequence of single-variable change immersion-assays with this pairing revealed that the pairing of $\mathrm{AmmMo} / \mathrm{HCl}$ is in fact unfavourable.

The polymeric sugar DEX was then investigated as a potential solid substrate for reagent delivery. Both bulk assays as well initial immersion assays revealed DEX as an apt substrate for this reaction, it does not interfere with the arsenic assay and it is fully transparent to light. The liquid-free arsenic assay proceeds with initial $\mathrm{HCl}$ introduction via a DEX pellet, and then $\mathrm{EV} / \mathrm{KIO}_{3}$ and $\mathrm{AmmMo}$ introduction via paper pads; 
the entire assay proceeds within a graduated Eppendorf vial, which is used to measure the volume of arsenic sample needed for the test. Following overnight reaction, the liquid-free $\mu$ PAD assay has an arsenic LOD of $78 \mu \mathrm{g} / \mathrm{L}$. This is based on computer-assisted calibration of reaction pad images, using normalised RGB $B$ values. 


\section{CONCLUSIONS AND FUTURE DIRECTIONS}

The fundamental purpose of this research is to evaluate the utility of microfluidics with regards to portable trace arsenic detection, and to explore the development of a simple portable arsenic sensor that can potentially be use for routine monitoring in arsenic affected regions. This work is motivated by the need for a better portable arsenic sensor for routine arsenic monitoring in regions such as Bangladesh, and the hope that the development of better technology may lead the lowering of their current arsenic MCL of 50 $\mu \mathrm{g} / \mathrm{L}$ down to the WHO-suggested standard of $10 \mu \mathrm{g} / \mathrm{L}$. The Gutzeit reaction-based portable arsenic field test kits that have thus far been used in Bangladesh are unreliable and unsafe. I believe that the ideal sensor is a complete microfluidic total analytical system ( $\mu$ TAS) for arsenic detection, with an LOD capable of sensing the WHO-recommended safe arsenic limit of $10 \mu \mathrm{g} / \mathrm{L}$. If successfully refined, the $\mu$ PAD assay that I have presented for arsenic detection can be commercialised for this purpose.

My research has analytical chemistry and microfluidics as its two main fields of study. An important contribution of my literature review, to both of these fields is the illustration that microfluidic technology has applications beyond biotechnology alone. Although we are not the first nor the only group to illustrate this approach, I am hopeful that my review can really emphasize this point and foster additional interest from the microfluidic community to such questions of study.

Furthermore, the findings of my review reveal that despite the many different potential methods for portable arsenic detection, colorimetric techniques are simplest and most practical for field applications. Therefore, my experimental efforts have been focused on the development of a simple, portable colorimetric sensor for arsenic based on molybdenum blue chemistry. Like the Gutzeit reaction, this is a classical, well-known reaction for arsenic detection; however, the lack of poisonous reagents or arsine gas generation in the molybdenum blue assay, renders it a much safer alternative for routine administration. Specifically focusing on the colorimetric arsenic assay developed by Morita and Kaneko, I have engineered a low-cost liquid-free portable sensor for arsenic using paper pads and dextran pellets as solid modes of reagent delivery.

Through computer-assisted calibration, the reported liquid-free sensor is capable of detecting arsenic levels down to $78 \mu \mathrm{g} / \mathrm{L}$. Furthermore, I have described a modification of reported protocol to potentially lower the limit of detection of the liquid-free arsenic assay down to $12 \mu \mathrm{g} / \mathrm{L}$, which is very closed to the WHO safe-contamination threshold. Once a fine-tuned protocol is developed, the true analytical ability of the assay must be evaluated against that of existing laboratory methods for arsenic detection such as HG-AAS and ICP-MS, as well as currently used field test kits. These existing technologies 
serve as benchmarks of the current standards of arsenic detection that are available for water quality monitoring.

Ideally, the eventual goal of this work is to lead to complete $\mu$ TAS for portable on-site arsenic detection. However, my current system addresses only of portion of this total process. The complete arsenic detection process consists of sample preparation, sample analysis, and signal acquisition; this current research focuses only on the sample analysis part of the picture. Thus far, my current experiments have simply been proof-of concept approaches in which our arsenic samples have been prepared with As(V) in deionized water. However, in order to analyse real samples using arsenic reaction, it is necessary to convert all arsenic species in solution to $\mathrm{As}(\mathrm{V})$ and to remove any interfering species such as $\mathrm{P}, \mathrm{Si}$, and $\mathrm{F}$. As described in the review, several approaches have been described it in the literature for this, but keeping in order to maintain the user-friendliness of my assay, it would be necessary to develop a simple, liquid-free method to achieve this.

Furthermore, for ease of use by local populations, it will be necessary to reconfigure my signal analysis process into an automated arsenic readout. For my paper-based sensor, portable image acquisition and analysis may potentially be carried out with something as simple a cell phone. Past research efforts in our group have focused on the development of a MATLAB code for the determination of arsenic concentration based on the colour intensity generated by existing test kits (Battaglia 2015). This code can easily be adapted for any colour-generating test strip, such as the one developed by myself. Additionally, to further increase the ease of use of the assay, this colour analysis readout can further be developed into a smartphone app.

Our research group currently has a collaboration with a medical doctor in Bangladesh, Dr. Uzzal Sikdar, Once the aforementioned pre- and post- assay needs are met, he will conduct field arsenic trials and evaluate the feasibility of this system for routine use by the local population. Should the system be deemed practical, then we can proceed with commercialisation of the complete arsenic $\mu$ TAS. Ideally, this final product will meet all of the criteria I have defined for the ideal portable sensor: high sensitivity and selectivity for arsenic; high reproducibility of detection; increased portability and robustness; reduced health and environmental risks; and affordability and ease of implementation. Already, my liquid-free arsenic sensor meets the latter three of these five criteria, in its current form; a positive nod in the direction of improved standards of routine arsenic monitoring in developing regions. 


\section{APPENDICES}

\section{General Methodological Features}

This section describes some basic methodological elements that are common to all of my experiments. Below, I describe my methods of solution preparation and paper device fabrication, as well as my general process of image acquisition and analysis.

The analysis of real samples by my methods requires two additional pre-processing steps: 1) removal of phosphate, silicate, and fluoride interferences; and 2) conversion of all arsenic in solution to the pentavalent form. As this initial work is intended to be a proof of principle, I have by-passed these steps and chosen to work with pure deionized (DI) water samples of varying amounts of $\operatorname{arsenic}(\mathrm{V})$ acid.

\section{Solution Preparation}

Arsenic solutions were prepared by serial dilution of the stock solution $\left(\mathrm{As}_{2} \mathrm{O}_{5}\right.$ in water, $1000 \mathrm{mg} / \mathrm{L}$ As(V) standard for ICP, Fluka). An initial dilution $(25.00 \mathrm{mg} / \mathrm{L})$ was used for the subsequent creation of a secondary stock $(1000 \mu \mathrm{g} / \mathrm{L})$, which was then used for all further serial dilutions $(500.0-1.000 \mu \mathrm{g} / \mathrm{L})$. All dilutions were prepared using de-ionised (DI) water. Volumetric glassware and micropipettes were used, where necessary. For all tests investigated, the method blank $(0 \mu \mathrm{g} / \mathrm{L})$ is represented by a pure solution of DI water.

The reagents required for the Morita and Kaneko assay are potassium iodate $\left(\mathrm{KIO}_{3} ; 0.0500 \mathrm{M}\right)$, ammonium heptamolybdate $\left(\left(\mathrm{NH}_{4}\right)_{6} \mathrm{Mo}_{7} \mathrm{O}_{24}\right)$, 'AmmMo'; $\left.0.0315 \mathrm{M}\right)$, hydrochloric acid $(\mathrm{HCl} ; 6 \mathrm{M})$, and ethyl violet $\left(\mathrm{C}_{31} \mathrm{H}_{42} \mathrm{ClN}_{3}\right.$, 'EV'; $\left.0.013 \mathrm{M}\right)$ (Morita \& Kaneko 2006a). All reagents were purchased in trace metals basis from Sigma-Aldrich. Solutions were prepared using volumetric glassware and DI water. 


\section{Paper Device Fabrication}

a.

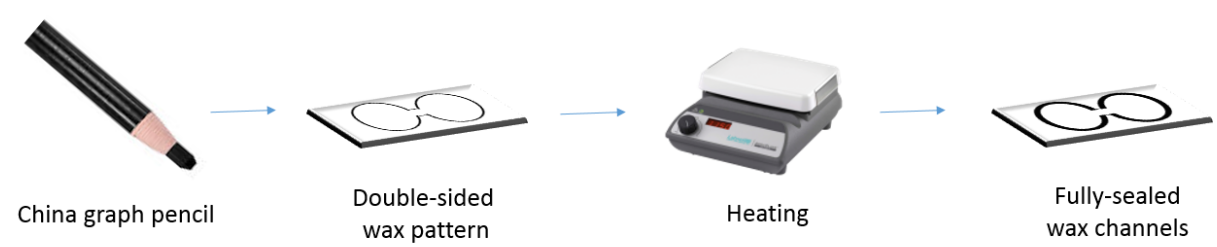

b.
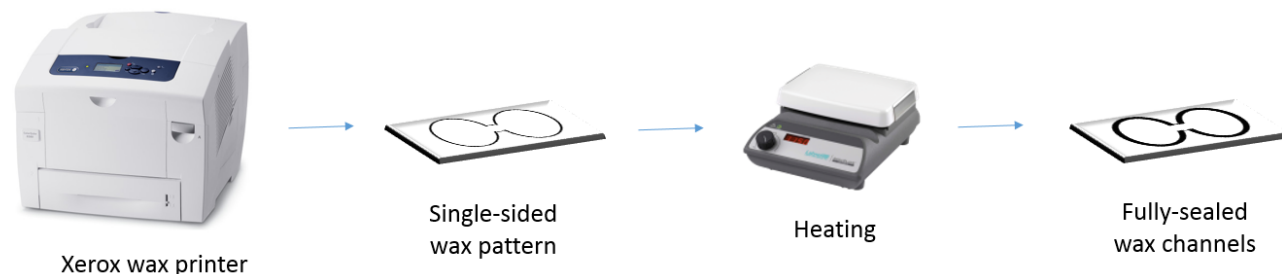

Figure A-1: Schematic diagram of the fabrication of paper devices; a. manual drawing of wax channels by use of China graph pencils, b. automated design of wax channels by use of a Xerox wax printer.

Paper devices and pads were fabricated by the creation of wax channels on cellulose chromatography paper (Whatman No. 1, Sigma-Aldrich). In initial devices, wax patterns were drawn manually (China graph pencils, Curry's Artists' Materials) on both sides of the paper. Drawn patterns were then converted into sealed channels by heating the paper $\left(1 \mathrm{~min} / \mathrm{side}, 150{ }^{\circ} \mathrm{C}\right.$, Fishier Scientific Isotemp digital hotplate). In later devices, wax geometries were first patterned using a computer software (Microsoft PowerPoint) and then single-sided printed onto the paper using a wax printer (Xerox ColorQube 8570). Printed patterns were then converted into sealed channels by heating the paper wax-side up ( 2 min, 175 $\left.{ }^{\circ} \mathrm{C}\right)$. FigureA-1 illustrates a schematic diagram of this process.

After wax channels are formed, the paper devices are cut apart and placed in Petri dishes; the reagent of interest is then pipetted into delineated zones and allowed to dry (1-4 hours). The required dry time is a function of the reagent used, reagent volume and ambient humidity. 


\section{Image Acquisition and Analysis}

a.

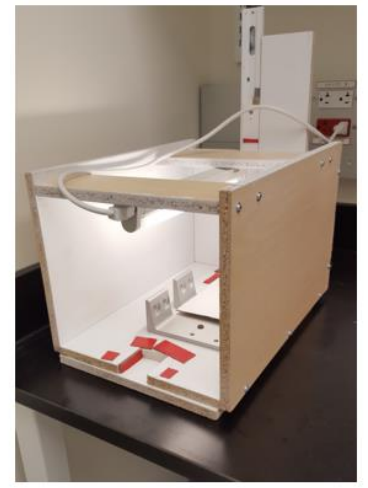

b.

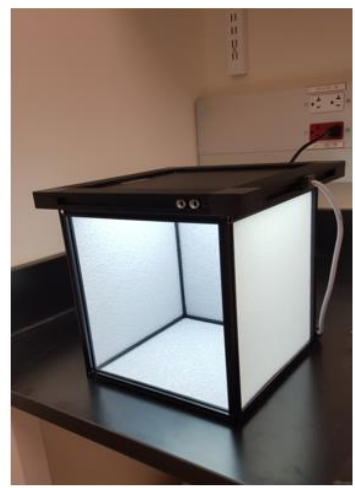

Figure A-2: Designated imaging boxes used with $\mu \mathrm{PAD}$ experiments. The light source in a. is a fluorescent bar of fixed brightness; the light source in $b$. is an adjustable power LED panel.

Table A-1: Nikon D90 exposure parameters used for image acquisition within the two different imaging boxes; exposure parameters were chosen in order to optimized the white balance of the generated images. The labeling of boxes A and B corresponds with that of FigureA-2.

\begin{tabular}{|l|l|l|}
\hline \multicolumn{1}{|c|}{ Parameter } & \multicolumn{1}{c|}{ Box A } & Box B \\
\hline Exposure Mode: & Manual & Manual \\
\hline Shutter Speed: & $1 / 160 \mathrm{sec}$ & $1 / 160 \mathrm{sec}$ \\
\hline Aperture: & f/10 & f/10 \\
\hline Exposure Comp.: & $+1 \mathrm{EV}$ & $+1 \mathrm{EV}$ \\
\hline Flash Comp.: & $0 \mathrm{EV}$ & $0 \mathrm{EV}$ \\
\hline Flexible Program: & 0 Steps & 0 Steps \\
\hline Metering: & Matrix & Matrix \\
\hline Flash Sync Mode: & Front Curtain & Front Curtain \\
\hline ISO Sensitivity: & ISO 640 & ISO 1000 \\
\hline White Balance: & $\begin{array}{l}\text { Fluorescent } \\
\text { (Cool-white fluorescent) }\end{array}$ & $\begin{array}{l}\text { Fluorescent } \\
\text { (High temp, mercury-vapor) }\end{array}$ \\
\hline
\end{tabular}

All images were acquired using a digital single-lens reflex camera (Nikon D90) with a macro lens (AF-S Micro NIKKOR $85 \mathrm{~mm}$ ). Two designated imaging boxes, pictured in Figure A-2, were utilised in an attempt to maintain constant ambient lighting across experimental images. Images were acquired using a computer software (Camera Control Pro v.2). Table lists the image acquisition parameters utilised with each imaging box; the parameters were so chosen in order to optimize the white balance of the experimental images. For the generation of plots, average RGB colour values were tabulated from histograms of the regions of interest (ImageJ). 


\section{REFERENCES}

Ackermann, K.R., Henkel, T. \& Popp, J., 2007. Quantitative online detection of low-concentrated drugs via a SERS microfluidic system. Chemphyschem, 8(18), pp.2665-70. Available at: http://www.ncbi.nlm.nih.gov/pubmed/18061914 [Accessed May 6, 2014].

Aleksic, J. et al., 2007. Development of a novel biosensor for the detection of arsenic in drinking water. IET Synthetic Biology, 1(1-2), pp.87-90.

Argos, M. et al., 2010. Arsenic exposure from drinking water, and all-cause and chronic-disease mortalities in Bangladesh (HEALS): a prospective cohort study. Lancet, 376(9737), pp.252-8. Available at: http://www.ncbi.nlm.nih.gov/pubmed/20646756 [Accessed October 3, 2013].

Arora, M., Megharaj, M. \& Naidu, R., 2009. Arsenic testing field kits: some considerations and recommendations. Environmental geochemistry and health, 31 Suppl 1, pp.45-8. Available at: http://www.ncbi.nlm.nih.gov/pubmed/19085061 [Accessed October 3, 2013].

Atefi, E., Mann, J.A. \& Tavana, H., 2014. Ultralow Interfacial Tensions of Aqueous Two-Phase Systems Measured Using Drop Shape. Langmuir, 30, pp.9631-9699.

Baastrup, R. et al., 2008. Arsenic in drinking-water and risk for cancer in Denmark. Environmental health perspectives, 116(2), pp.231-7. Available at:

http://www.pubmedcentral.nih.gov/articlerender.fcgi?artid=2235208\&tool=pmcentrez\&rendertype= abstract [Accessed April 12, 2014].

Baron, R. et al., 2007. Electrochemical detection of arsenic on a gold nanoparticle array. Russian Journal of Physical Chemistry A, 81(9), pp.1443-1447. Available at: http://link.springer.com/10.1134/S003602440709018X [Accessed February 10, 2014].

Barros, H. et al., 2010. Determination of arsenic in water samples by Total Reflection X-Ray Fluorescence using pre-concentration with alumina. Spectrochimica Acta Part B, 65(6), pp.489-492. Available at: http://linkinghub.elsevier.com/retrieve/pii/S0584854710001151 [Accessed March 24, 2014].

Barrows, J.N., Jameson, G.B. \& Pope, M.T., 1985. Structure of a Heteropoly Blue. The Four-Electron Reduced B-12-Molybdophosphate Anion. Journal of the American Chemical Society, 107, pp.17711773 .

Battaglia, B.J., 2015. Refining point of care technology: quantification in arsenic field test kits. Ryerson University, Toronto, Canada.

Behari, J.R. \& Prakash, R., 2006. Determination of total arsenic content in water by atomic absorption spectroscopy (AAS) using vapour generation assembly (VGA). Chemosphere, 63(1), pp.17-21. Available at: http://www.ncbi.nlm.nih.gov/pubmed/16213544 [Accessed January 11, 2014].

Bethany, C. et al., 2013. Dehydrated aqueous two-phase system micro-domains retain their shape upon rehydration to allow patterned reagent delivery to cells. Journal of Materials Chemistry B, 1(44), pp.6020-6026. 
Bindiganavale, G. et al., 2012. Digital Microfluidic Device for Hotspot Cooling in ICS Using Electrowetting on Dielectric. In ASME 2012 Third International Conference on Micro/Nanoscale Heat and Mass Transfer. pp. 39-42.

Bose, U., Rahman, M. \& Alamgir, M., 2011. Arsenic toxicity and speciation analysis in ground water samples: a review of some techniques. International Journal of Chemical Technology, 3(1), pp.1425.

Brindle, I.D., 2007. Vapour-generation analytical chemistry: from Marsh to multimode sampleintroduction system. Analytical and bioanalytical chemistry, 388(4), pp.735-41. Available at: http://www.ncbi.nlm.nih.gov/pubmed/17333151 [Accessed May 16, 2014].

Brouwer, P., 2010. Theory of XRF, Lelyweg, Almelo, the Netherlands.

Buffi, N. et al., 2011. Development of a microfluidics biosensor for agarose-bead immobilized Escherichia coli bioreporter cells for arsenite detection in aqueous samples. Lab on a chip, 11(14), pp.2369-77. Available at: http://www.ncbi.nlm.nih.gov/pubmed/21614381 [Accessed February 1, 2014].

Chan, A.S. et al., 2014. A simple microfluidic chip design for fundamental bioseparation. Journal of analytical methods in chemistry, pp.1-6. Available at: http://www.pubmedcentral.nih.gov/articlerender.fcgi?artid=3910460\&tool=pmcentrez\&rendertype= abstract.

Chang, H. et al., 2014. Modified nanoporous membranes on centrifugal microfluidic platforms for detecting heavy metal ions. , 18, pp.685-690.

Channg, Y.-C. \& Reid, J.F., 1996. RGB calibration for color image analysis in machine vision. IEEE transactions on image processing, 5(10), pp.1414-1422.

Chen, C.J. et al., 1986. A retrospective study on malignant neoplasms of bladder, lung and liver in blackfoot disease endemic area in Taiwan. British journal of cancer, 53(3), pp.399-405. Available at:

http://www.pubmedcentral.nih.gov/articlerender.fcgi?artid=2001352\&tool=pmcentrez\&rendertype= abstract.

Chen, M.-L., Ma, L.-Y. \& Chen, X.-W., 2014. New procedures for arsenic speciation: A review. Talanta, 125, pp.78-86. Available at: http://linkinghub.elsevier.com/retrieve/pii/S0039914014001350 [Accessed April 8, 2014].

Chen, Z. et al., 2010. Sensitive detection of metals in water using laser-induced breakdown spectroscopy on wood sample substrates. Applied Optics, 49(13), p.C87. Available at: http://www.opticsinfobase.org/abstract.cfm?URI=ao-49-13-C87.

Cicero, L.A., 2009. Scientists solve puzzle of arsenic-poisoning crisis in Asia. Stanford Report. 
Cosnier, S. et al., 2006. Specific determination of As(V) by an acid phosphatase-polyphenol oxidase biosensor. Analytical chemistry, 78(14), pp.4985-9. Available at:

http://www.ncbi.nlm.nih.gov/pubmed/16841921.

Cybulski, J., Clements, J. \& Prakash, M., 2014. Foldscope : Origami-based paper microscope. PLoS ONE, $9(6)$, pp.1-36.

Dai, X. et al., 2004. Anodic stripping voltammetry of arsenic(III) using gold nanoparticle-modified electrodes. Analytical chemistry, 76(19), pp.5924-9. Available at: http://www.ncbi.nlm.nih.gov/pubmed/15456316.

Dai, X. \& Compton, R.G., 2006. Detection of As(III) via oxidation to As(V) using platinum nanoparticle modified glassy carbon electrodes: arsenic detection without interference from copper. The Analyst, 131(4), pp.516-21. Available at: http://www.ncbi.nlm.nih.gov/pubmed/16568168 [Accessed April $27,2014]$.

Das, J. et al., 2014. Low-cost field test kits for arsenic detection in water. Journal of environmental science and health. Part A, Toxic/hazardous substances \& environmental engineering, 49(1), pp.108-15. Available at: http://www.ncbi.nlm.nih.gov/pubmed/24117090 [Accessed January 23, 2014].

Dasgupta, P.K. et al., 2002. Photometric measurement of trace As(III) and As(V) in drinking water. Talanta, 58(1), pp.153-64. Available at: http://www.ncbi.nlm.nih.gov/pubmed/18968742.

Deng, F. et al., 2013. Determination of trace total inorganic arsenic by hydride generation atomic fluorescence spectrometry after solid phase extraction-preconcentration on aluminium hydroxide gel. Microchimica Acta, 180(5-6), pp.509-515. Available at:

http://link.springer.com/10.1007/s00604-013-0941-7 [Accessed January 19, 2014].

Deshpande, L.S. \& Pande, S.P., 2005. Development of arsenic testing field kit--a tool for rapid on-site screening of arsenic contaminated water sources. Environmental monitoring and assessment, 101(13), pp.93-101. Available at: http://www.ncbi.nlm.nih.gov/pubmed/15736878.

Dhar, R.K. et al., 2004. A rapid colorimetric method for measuring arsenic concentrations in groundwater. Analytica Chimica Acta, 526(2), pp.203-209. Available at: http://linkinghub.elsevier.com/retrieve/pii/S0003267004012528 [Accessed February 2, 2014].

Diesel, E., Schreiber, M. \& van der Meer, J.R., 2009. Development of bacteria-based bioassays for arsenic detection in natural waters. Analytical and bioanalytical chemistry, 394(3), pp.687-93. Available at: http://www.ncbi.nlm.nih.gov/pubmed/19377836 [Accessed January 9, 2014].

Dorfman, K.D. et al., 2013. Beyond Gel Electrophoresis : Microfluidic Separations, Fluorescence Burst Analysis , and DNA Stretching. Chemical Reviews, 113, pp.2584-2667.

Fadaei, H., Scarff, B. \& Sinton, D., 2011. Rapid Microfluidics-Based Measurement of CO 2 Diffusivity in Bitumen. Energy and Fuels, 25, pp.4829-4835. 
Feeney, R. \& Kounaves, S., 2000. On-site analysis of arsenic in groundwater using a microfabricated gold ultramicroelectrode array. Analytical chemistry, 72(10), pp.2222-8. Available at:

http://www.ncbi.nlm.nih.gov/pubmed/10845367.

Feeney, R. \& Kounaves, S.P., 2002. Voltammetric measurement of arsenic in natural waters. Talanta, 58(1), pp.23-31. Available at: http://www.ncbi.nlm.nih.gov/pubmed/18968731.

Ferreccio, C. et al., 2000. Lung cancer and arsenic concentrations in drinking water in Chile. Epidemiology (Cambridge, Mass.), 11(6), pp.673-9. Available at: http://www.ncbi.nlm.nih.gov/pubmed/11055628.

Flanagan, S. V, Johnston, R.B. \& Zheng, Y., 2012. Arsenic in tube well water in Bangladesh: health and economic impacts and implications for arsenic mitigation. Bulletin of the World Health Organization, 90(11), pp.839-46. Available at:

http://www.pubmedcentral.nih.gov/articlerender.fcgi?artid=3506399\&tool=pmcentrez\&rendertype= abstract.

Forsberg, G. et al., 1975. Determination of arsenic by anodic stripping voltammetry and differential pulse anodic stripping voltammetry. Analytical Chemistry, 47(9), pp.1586-1592. Available at: http://pubs.acs.org/doi/abs/10.1021/ac60359a057.

Forzani, E.S. et al., 2007. Detection of arsenic in groundwater using a surface plasmon resonance sensor. Sensors and Actuators B: Chemical, 123(1), pp.82-88. Available at: http://linkinghub.elsevier.com/retrieve/pii/S0925400506005247 [Accessed November 24, 2013].

Francesconi, K. a \& Kuehnelt, D., 2004. Determination of arsenic species: a critical review of methods and applications, 2000-2003. The Analyst, 129(5), pp.373-95. Available at:

http://www.ncbi.nlm.nih.gov/pubmed/15116227.

Fua, E. et al., 2012. Transport in two-dimensional paper networks. Microfluidics and Nanofluidics, 10(1), pp.29-35.

Fujiwara, K., Watanabe, Y. \& Fuwa, K., 1982. Gas-Phase Chemilurninescence with Ozone Oxidation for the Determination of Arsenic, Antimony, Tin, and Selenium. Analytical chemistry, 54, pp.125-128.

Galopin, E. et al., 2007. SPR biosensing coupled to a digital microfluidic microstreaming system. Biosensors \& bioelectronics, 23(5), pp.746-50. Available at:

http://www.ncbi.nlm.nih.gov/pubmed/17884436 [Accessed May 4, 2014].

Gao, C. et al., 2013. Electrochemical detection of arsenic(III) completely free from noble metal: Fe3O4 microspheres-room temperature ionic liquid composite showing better performance than gold. Analytical chemistry, 85(5), pp.2673-80. Available at: http://www.ncbi.nlm.nih.gov/pubmed/23374085.

García, A. et al., 2011. Mobile phone platform as portable chemical analyzer. Sensors and Actuators B: Chemical, 156(1), pp.350-359. Available at:

http://linkinghub.elsevier.com/retrieve/pii/S092540051100342X [Accessed March 20, 2014]. 
George, C.M. et al., 2012. Impact on arsenic exposure of a growing proportion of untested wells in Bangladesh. Environmental health, 11(1), p.7. Available at:

http://www.pubmedcentral.nih.gov/articlerender.fcgi?artid=3334680\&tool=pmcentrez\&rendertype= abstract [Accessed December 18, 2014].

Ghosh, S.K. et al., 2002. Silver and Gold Nanocluster Catalyzed Reduction of Methylene Blue by Arsine in a Micellar Medium. Langmuir, 18(23), pp.8756-8760. Available at: http://pubs.acs.org/doi/abs/10.1021/la0201974.

Godwal, Y. et al., 2008. Development of laser-induced breakdown spectroscopy for microanalysis applications. Laser and Particle Beams, 26(01), pp.95-103. Available at: http://www.journals.cambridge.org/abstract_S0263034608000128 [Accessed May 7, 2014].

Godwal, Y. et al., 2008. Elemental analysis using micro laser-induced breakdown spectroscopy (microLIBS) in a microfluidic platform. Optics express, 16(17), pp.12435-45. Available at: http://www.ncbi.nlm.nih.gov/pubmed/18711480.

Gomez, F.A., 2011. Microfliuidics in Protein Chromatography. In Methods in molecular biology. Springer, pp. 137-150.

Gómez-Ariza, J.L. et al., 2000. A comparison between ICP-MS and AFS detection for arsenic speciation in environmental samples. Talanta, 51(2), pp.257-68. Available at: http://www.ncbi.nlm.nih.gov/pubmed/18967857.

Gómez-sjöberg, R., Morisette, D.T. \& Bashir, R., 2005. Impedance Microbiology-on-a-Chip : Microfluidic Bioprocessor for Rapid Detection of Bacterial Metabolism. Journal of Microelectromechanical Systems, 14(4), pp.829-838.

Gong, M.M. et al., 2012. Hand-powered microfluidics: A membrane pump with a patient-to-chip syringe interface. Biomicrofluidics, 6(4), pp.1-13.

Gong, M.M. et al., 2012. Hand-powered microfluidics: A membrane pump with a patient-to-chip syringe interface. Biomicrofluidics, 6(4), p.44102. Available at: http://www.pubmedcentral.nih.gov/articlerender.fcgi?artid=3487897\&tool=pmcentrez\&rendertype= abstract.

Guijt, R.M. et al., 2004. Conductivity detection for conventional and miniaturised capillary electrophoresis systems. Electrophoresis, 25(23-24), pp.4032-57. Available at: http://www.ncbi.nlm.nih.gov/pubmed/15597418 [Accessed January 29, 2014].

Haider, a. F.M.Y. et al., 2014. Detection of trace amount of arsenic in groundwater by laser-induced breakdown spectroscopy and adsorption. Optics \& Laser Technology, 56, pp.299-303. Available at: http://linkinghub.elsevier.com/retrieve/pii/S0030399213003277 [Accessed March 22, 2014].

Hashem, A. et al., 2011. High Sensitivity Arsenic Analyzer Based on Liquid-reagent-free Hydride Generation and Chemiluminescence Detection for On-site Water Analysis. Analytical sciences, 27, pp.733-738. 
Hettiarachchi, K. et al., 2007. On-chip generation of microbubbles as a practical technology for manufacturing contrast agents for ultrasonic imaging. Lab on a chip, 7(4), pp.463-8. Available at: http://www.pubmedcentral.nih.gov/articlerender.fcgi?artid=2253663\&tool=pmcentrez\&rendertype= abstract [Accessed March 28, 2014].

Hopenhayn-Rich, C., Biggs, M.L. \& Smith, a H., 1998. Lung and kidney cancer mortality associated with arsenic in drinking water in Córdoba, Argentina. International journal of epidemiology, 27(4), pp.561-9. Available at: http://www.ncbi.nlm.nih.gov/pubmed/9758107.

Hossain, S.M.Z. et al., 2012. Multiplexed paper test strip for quantitative bacterial detection. Analytical and bioanalytical chemistry, 403(6), pp.1567-76. Available at: http://www.ncbi.nlm.nih.gov/pubmed/22526653 [Accessed September 23, 2013].

Hossain, S.M.Z. \& Brennan, J.D., 2011. $\beta$-Galactosidase-Based Colorimetric Paper Sensor for Determination. Analytical Chemistry, 83, pp.8772-8778.

Hung, D.Q., Nekrassova, O. \& Compton, R.G., 2004. Analytical methods for inorganic arsenic in water: a review. Talanta, 64, pp.269-277. Available at: http://www.ncbi.nlm.nih.gov/pubmed/18969599.

Hylton, K. \& Mitra, S., 2008. A microfluidic hollow fiber membrane extractor for arsenic(V) detection. Analytica chimica acta, 607(1), pp.45-9. Available at: http://www.ncbi.nlm.nih.gov/pubmed/18155408 [Accessed February 1, 2014].

Ibraheem, N.A. et al., 2012. Understanding Color Models : A Review. Journal of Science and Technology, 2(3), pp.265-275.

Jakariya, M. et al., 2007. Screening of arsenic in tubewell water with field test kits: evaluation of the method from public health perspective. The Science of the total environment, 379(2-3), pp.167-75. Available at: http://www.ncbi.nlm.nih.gov/pubmed/17258792 [Accessed October 3, 2013].

Kalluri, J.R. et al., 2009. Use of gold nanoparticles in a simple colorimetric and ultrasensitive dynamic light scattering assay: selective detection of arsenic in groundwater. Angewandte Chemie (International ed. in English), 48(51), pp.9668-71. Available at: http://www.ncbi.nlm.nih.gov/pubmed/19937875 [Accessed February 10, 2014].

Karlsson, R., Michaelsson, a \& Mattsson, L., 1991. Kinetic analysis of monoclonal antibody-antigen interactions with a new biosensor based analytical system. Journal of immunological methods, 145(1-2), pp.229-40. Available at: http://www.ncbi.nlm.nih.gov/pubmed/1765656.

Katsoyiannis, I. a \& Zouboulis, A.I., 2002. Removal of arsenic from contaminated water sources by sorption onto iron-oxide-coated polymeric materials. Water research, 36(20), pp.5141-55. Available at: http://www.ncbi.nlm.nih.gov/pubmed/12448563.

Kearns, J. \& Tyson, J., 2012. Improving the accuracy and precision of an arsenic field test kit: increased reaction time and digital image analysis. Analytical Methods, 4(6), p.1693. Available at: http://xlink.rsc.org/?DOI=c2ay05655k [Accessed December 18, 2014]. 
Khairy, M. et al., 2010. Gold Nanoparticle Modified Screen Printed Electrodes for the Trace Sensing of Arsenic(III) in the Presence of Copper(II). Electroanalysis, 22(21), pp.2496-2501. Available at: http://doi.wiley.com/10.1002/elan.201000226 [Accessed October 12, 2014].

Kim, U. et al., 2013. Development of low-cost plastic microfluidic sensors toward rapid and point-of-use detection of arsenic in drinking water for global health. 2013 IEEE Biomedical Circuits and Systems Conference, BioCAS 2013, pp.113-117.

Kinniburgh, D. \& Smedley, P., 2001. Arsenic contamination of groundwater in Bangladesh, Available at: http://nora.nerc.ac.uk/id/eprint/11986 [Accessed September 10, 2014].

Kinniburgh, D.G. \& Kosmus, W., 2002. Arsenic contamination in groundwater: some analytical considerations. Talanta, 58(1), pp.165-80. Available at: http://www.ncbi.nlm.nih.gov/pubmed/18968743.

Klaue, B. \& Blum, J.D., 1999. Trace Analyses of Arsenic in Drinking Water by Inductively Coupled Plasma Mass Spectrometry: High Resolution versus Hydride Generation spectrometer ( ICPMS ) was applied to the determination chloride interference, the accompanying reduction in. Analytical Chemistry, 71(7), pp.1408-1414.

Kundu, S. et al., 2005. Ion-associate of arsenic ( V ) -salicylic acid chelate with methylene blue in toluene: Application for arsenic quantification. Indian Journal of Chemistry, 44(October), pp.2030 2033.

Kundu, S. et al., 2002. Spectrophotometric determination of arsenic via arsine generation and in-situ colour bleaching of methylene blue (MB) in micellar medium. Talanta, 58(5), pp.935-42. Available at: http://www.ncbi.nlm.nih.gov/pubmed/18968826.

Kurita, R. et al., 2006. On-chip enzyme immunoassay of a cardiac marker using a microfluidic device combined with a portable surface plasmon resonance system. Analytical chemistry, 78(15), pp.5525-31. Available at: http://www.ncbi.nlm.nih.gov/pubmed/16878891.

Kurniawan, T.A., Sillanpää, M.E.T. \& Sillanpää, M., 2012. Nanoadsorbents for Remediation of Aquatic Environment: Local and Practical Solutions for Global Water Pollution Problems. Critical Reviews in Environmental Science and Technology, 42(12), pp.1233-1295. Available at: http://www.tandfonline.com/doi/abs/10.1080/10643389.2011.556553 [Accessed September 21, 2013].

Lamm, S.H., Engel, A. \& Kruse, A.B., 2004. Arsenic in drinking water and bladder cancer mortality in the United States: an analysis based on 133 U.S. countries and 30-years of observation. Journal of Occupational and Environmental Medicine, 46, pp.298-306. Available at: http://content.wkhealth.com/linkback/openurl?sid=WKPTLP:landingpage \&an=00043764200410000-00002 [Accessed April 22, 2014].

Levine, H., Rowe, J.J. \& Grimaldi, F.S., 1954. The Molybdenum Blue Reaction and the Determination of Phosphorus in Waters Containing Arsenic, Silicon, and Germanium. Science (New York, N.Y.), 119(3088), pp.327-8. Available at: http://www.ncbi.nlm.nih.gov/pubmed/17754335. 
Li, F. et al., 2005. Speciation analysis of inorganic arsenic by microchip capillary electrophoresis coupled with hydride generation atomic fluorescence spectrometry. Journal of Chromatography A, 1081(2), pp.232-237. Available at: http://linkinghub.elsevier.com/retrieve/pii/S0021967305010290 [Accessed March 24, 2014].

Li, H.-F. \& Lin, J.-M., 2009. Applications of microfluidic systems in environmental analysis. Analytical and bioanalytical chemistry, 393(2), pp.555-67. Available at: http://www.ncbi.nlm.nih.gov/pubmed/18972106 [Accessed February 1, 2014].

Linares, P. \& Castro, M.D.L. De, 1986. Flow Injection Analysis of Binary and Ternary Mixtures of Arsenite, Arsenate, and Phosphate. Analytical chemistry, 58, pp.120-124.

Lindberg, A.-L. et al., 2007. Evaluation of the three most commonly used analytical methods for determination of inorganic arsenic and its metabolites in urine. Toxicology letters, 168(3), pp.310-8. Available at: http://www.ncbi.nlm.nih.gov/pubmed/17174488 [Accessed January 9, 2014].

Lu, Y. et al., 2009. Low cost, portable detection of gold nanoparticle-labeled microfluidic immunoassay with camera cell phone. Electrophoresis, 30(4), pp.579-82. Available at: http://www.ncbi.nlm.nih.gov/pubmed/19170056 [Accessed April 26, 2014].

Luo, Y., Yu, F. \& Zare, R.N., 2008. Microfluidic device for immunoassays based on surface plasmon resonance imaging. Lab on a chip, 8(5), pp.694-700. Available at: http://www.ncbi.nlm.nih.gov/pubmed/18432338 [Accessed April 29, 2014].

Luong, J.H.T., Majid, E. \& Male, K.B., 2007. Analytical Tools for Monitoring Arsenic in the Environment. The Open Analytical Chemistry Journal, 1(1), pp.7-14. Available at: http://www.benthamopen.org/pages/content.php?TOACJ/2007/00000001/00000001/7TOACJ.SGM.

Majid, E. et al., 2006. Electrochemical determination of arsenite using a gold nanoparticle modified glassy carbon electrode and flow analysis. Analytical chemistry, 78(3), pp.762-9. Available at: http://www.ncbi.nlm.nih.gov/pubmed/16448049.

Male, K.B. et al., 2007. Biosensor for Arsenite Using Arsenite Oxidase and Multiwalled Carbon Nanotube Modified Electrodes lybdenum-containing arsenite oxidase, prepared from the. Analytical chemistry, 79(20), pp.7831-7837.

Manz, A., Graber, N. \& Widmer, H.M., 1990. Miniaturized total chemcial analysis systems: a novel concept for chemical sensing. Sensors and Actuators B Chemical, 1, pp.244-248.

Marquardt, B.J., Goode, S.R. \& Angel, S.M., 1996. In Situ Determination of Lead in Paint by LaserInduced Breakdown Spectroscopy Using a Fiber-Optic Probe. Analytical Chemistry, 68(6), pp.977981. Available at: http://pubs.acs.org/doi/abs/10.1021/ac950828h.

Martinez, A.W. et al., 2010. Diagnostics for the developing world: microfluidic paper-based analytical devices. Analytical chemistry, 82(1), pp.3-10. Available at: http://www.ncbi.nlm.nih.gov/pubmed/20000334. 
Martinez, A.W. et al., 2008. Simple telemedicine for developing regions: camera phones and paper-based microfluidic devices for real-time, off-site diagnosis. Analytical chemistry, 80(10), pp.3699-707. Available at:

http://www.pubmedcentral.nih.gov/articlerender.fcgi?artid=3761971\&tool=pmcentrez\&rendertype= abstract.

Matysik, F.-M., 2003. Miniaturization of electroanalytical systems. Analytical and bioanalytical chemistry, 375(1), pp.33-5. Available at: http://www.ncbi.nlm.nih.gov/pubmed/12520433 [Accessed December 14, 2014].

Mayo, J.T. et al., 2007. The effect of nanocrystalline magnetite size on arsenic removal. Science and Technology of Advanced Materials, 8(1-2), pp.71-75. Available at: http://stacks.iop.org/14686996/8/i=1-2/a=A13?key=crossref.6d1cf6efd58428eeea35ee1d350a3b60 [Accessed October 1, 2013].

Mays, D.E. \& Hussam, A., 2009. Voltammetric methods for determination and speciation of inorganic arsenic in the environment--a review. Analytica chimica acta, 646(1-2), pp.6-16. Available at: http://www.ncbi.nlm.nih.gov/pubmed/19523550 [Accessed April 10, 2014].

Melamed, D., 2005. Monitoring arsenic in the environment: a review of science and technologies with the potential for field measurements. Analytica Chimica Acta, 532(1), pp.1-13. Available at: http://linkinghub.elsevier.com/retrieve/pii/S0003267004013996 [Accessed November 24, 2013].

Mentele, M.M. et al., 2012. Microfluidic Paper-Based Analytical Device for Particulate Metals. , 84, pp.4474-4480.

Merulla, D. et al., 2013. Bioreporters and biosensors for arsenic detection. Biotechnological solutions for a world-wide pollution problem. Current opinion in biotechnology, 24(3), pp.534-41. Available at: http://www.ncbi.nlm.nih.gov/pubmed/22999825 [Accessed April 30, 2014].

Morales, K.H. et al., 2000. Risk of internal cancers from arsenic in drinking water. Environmental health perspectives, 108(7), pp.655-61. Available at:

http://www.pubmedcentral.nih.gov/articlerender.fcgi?artid=1240527\&tool=pmcentrez\&rendertype= abstract.

Morita, K. \& Kaneko, E., 2006a. Spectrophotometric determination of arsenic in water samples based on micro particle formation of ethyl violet-molybdoarsenate. Analytical sciences : the international journal of the Japan Society for Analytical Chemistry, 22(8), pp.1085-9. Available at: http://www.ncbi.nlm.nih.gov/pubmed/16896247.

Morita, K. \& Kaneko, E., 2006b. Spectrophotometric determination of trace arsenic in water samples using a nanoparticle of ethyl violet with a molybdate-iodine tetrachloride complex as a probe for molybdoarsenate. Analytical chemistry, 78(22), pp.7682-8. Available at: http://www.ncbi.nlm.nih.gov/pubmed/17105159.

Mukherjee, A. et al., 2005. Comment on "Reliability of a commercial kit to test groundwater for arsenic in Bangladesh". Environmental science \& technology, 39(14), pp.5501-2; author reply 5503-4. Available at: http://www.ncbi.nlm.nih.gov/pubmed/16082988. 
Mulvihill, M. et al., 2008. Surface-Enhanced Raman Spectroscopy for Trace Arsenic Detection in Contaminated Water. Angewandte Chemie, 120(34), pp.6556-6560. Available at:

http://doi.wiley.com/10.1002/ange.200800776 [Accessed October 2, 2013].

Nath, P., Arun, K. \& Chanda, N., 2014. A paper based microfluidic device for the detection of arsenic using a gold nanosensor. RSC Advances, 4, pp.59558-59561. Available at: http://dx.doi.org/10.1039/C4RA12946F.

Nie, S., 1997. Probing Single Molecules and Single Nanoparticles by Surface-Enhanced Raman Scattering. Science, 275(5303), pp.1102-1106. Available at: http://www.sciencemag.org/cgi/doi/10.1126/science.275.5303.1102 [Accessed April 30, 2014].

Oh, C.S., 1999. Microfluidic Electrophoresis Device.

Okazaki, T. et al., 2015. Analytical Methods Visual colorimetry for determination of trace arsenic in groundwater based on improved molybdenum blue spectrophotometry. Analytical Methods, 7, pp.2794-2799. Available at: http://dx.doi.org/10.1039/C4AY03021D.

Pande, S.P., Deshpande, L.S. \& Kaul, S.N., 2001. Laboratory and field assessment of arsenic testing field kits in Bangladesh and West Bengal, India. Environmental monitoring and assessment, 68(1), pp.118. Available at: http://www.ncbi.nlm.nih.gov/pubmed/11336408.

Park, T.S. et al., 2013. Smartphone quantifies Salmonella from paper microfluidics. Lab on a chip, 13(24), pp.4832-40. Available at: http://www.ncbi.nlm.nih.gov/pubmed/24162816 [Accessed March 20, 2014].

Petrusevski, B. \& Sharma, S., 2007. Arsenic in Drinking Water. IRC International Water and Sanitation Centre.

Plinius Secondus (Pliny), G., Historia Naturae, Book IX,

Prest, J. et al., 2003. Miniaturised isotachophoretic analysis of inorganic arsenic speciation using a planar polymer chip with integrated conductivity detection. Journal of Chromatography A, 990(1-2), pp.325-334. Available at: http://linkinghub.elsevier.com/retrieve/pii/S0021967302019684 [Accessed February 27, 2014].

Prest, J.E. et al., 2005. Inorganic Arsenic and Selenium Determination Using Miniaturised Isotachophoresis. Microchimica Acta, 151(3-4), pp.223-230. Available at: http://link.springer.com/10.1007/s00604-005-0403-y [Accessed February 27, 2014].

Qian, X.-M. \& Nie, S.M., 2008. Single-molecule and single-nanoparticle SERS: from fundamental mechanisms to biomedical applications. Chemical Society reviews, 37(5), pp.912-20. Available at: http://www.ncbi.nlm.nih.gov/pubmed/18443676 [Accessed May 6, 2014].

Rahman, M.M. et al., 2002. Effectiveness and reliability of arsenic field testing kits: are the million dollar screening projects effective or not? Environmental science \& technology, 36(24), pp.5385-94. Available at: http://www.ncbi.nlm.nih.gov/pubmed/12521165. 
Rajkumar, M., Thiagarajan, S. \& Chen, S., 2011. Electrochemical Detection of Arsenic in Various Water Samples. International Journal of Electrochemical Science, 6, pp.3164-3177.

Renedo, O.D., Alonso-Lomillo, M. a \& Martínez, M.J.A., 2007. Recent developments in the field of screen-printed electrodes and their related applications. Talanta, 73(2), pp.202-19. Available at: http://www.ncbi.nlm.nih.gov/pubmed/19073018 [Accessed August 21, 2014].

Roje, V., Mikac, N. \& Kniewald, G., 2007. Determination of Arsenic and Other Trace Elements in Bottled Waters by High Resolution Inductively Coupled Plasma Mass Spectrometry. Croatica Chemica Acta, 80(1), pp.91-100.

Rosenfeld, T. \& Bercovici, M., 2014. 1000-Fold Sample Focusing on Paper-Based Microfluidic Devices. Lab on a chip, 14(23), pp.4465-74. Available at: http://www.ncbi.nlm.nih.gov/pubmed/25256832 [Accessed December 18, 2014].

Rothert, A. et al., 2005. Whole-cell-reporter-gene-based biosensing systems on a compact disk microfluidics platform. Analytical biochemistry, 342(1), pp.11-9. Available at: http://www.ncbi.nlm.nih.gov/pubmed/15958175 [Accessed March 1, 2014].

Ruano-López, J.M. et al., 2009. The SmartBioPhone, a point of care vision under development through two European projects: OPTOLABCARD and LABONFOIL. Lab on a chip, 9(11), pp.1495-9. Available at: http://www.ncbi.nlm.nih.gov/pubmed/19458852 [Accessed April 7, 2014].

Rupasinghe, T. et al., 2004. Determination of arsenic by pervaporation-flow injection hydride generation and permanganate spectrophotometric detection. Analytica Chimica Acta, 510(2), pp.225-230. Available at: http://linkinghub.elsevier.com/retrieve/pii/S0003267004000303 [Accessed February 8, 2014].

Rupasinghe, T. et al., 2001. Pervaporation-flow injection determination of arsenic based on hydride generation and the molybdenum blue reaction. Analytica Chimica Acta, 445(2), pp.229-238. Available at: http://linkinghub.elsevier.com/retrieve/pii/S0003267001012569.

Rusak, D. a. et al., 1997. Fundamentals and Applications of Laser-Induced Breakdown Spectroscopy. Critical Reviews in Analytical Chemistry, 27(4), pp.257-290. Available at: http://www.tandfonline.com/doi/abs/10.1080/10408349708050587 [Accessed May 6, 2014].

Safarzadeh-Amiri, a et al., 2011. Validation of analysis of arsenic in water samples using Wagtech Digital Arsenator. The Science of the total environment, 409(13), pp.2662-7. Available at: http://www.ncbi.nlm.nih.gov/pubmed/21497375 [Accessed October 3, 2013].

Salimi, A. et al., 2008. Electrochemical detection of trace amount of arsenic(III) at glassy carbon electrode modified with cobalt oxide nanoparticles. Sensors and Actuators B: Chemical, 129(1), pp.246-254. Available at: http://linkinghub.elsevier.com/retrieve/pii/S0925400507006211 [Accessed March 25, 2014].

Salman, M. et al., 2012. Micro-determination of arsenic in aqueous samples by image scanning and computational quantification. Analytical Methods, 4, pp.242-246. 
Samanta, G. et al., 1999. Flow Injection Hydride Generation Atomic Absorption Spectrometry for Determination of Arsenic in Water and Biological Samples from Arsenic-Affected Districts of West Bengal, India, and Bangladesh. Microchemical Journal, 62(1), pp.174-191. Available at: http://linkinghub.elsevier.com/retrieve/pii/S0026265X99917137.

Sanger, C.R. \& Black, O.F., 1907. The Quantitative Determination of Arsenic by the Gutzeit Method. Proceedings of the American Academy of Arts and Sciences, 43(8), pp.297-324.

Sankararamakrishnan, N. et al., 2008. Evaluation of two commercial field test kits used for screening of groundwater for arsenic in Northern India. The Science of the total environment, 401(1-3), pp.1627. Available at: http://www.ncbi.nlm.nih.gov/pubmed/18514262 [Accessed April 25, 2014].

Sanllorente-Méndez, S., Domínguez-Renedo, O. \& Arcos-Martínez, M.J., 2010. Immobilization of acetylcholinesterase on screen-printed electrodes. Application to the determination of arsenic(III). Sensors (Basel, Switzerland), 10(3), pp.2119-28. Available at: http://www.pubmedcentral.nih.gov/articlerender.fcgi?artid=3264471\&tool=pmcentrez\&rendertype= abstract [Accessed March 16, 2014].

Sbarato, V.M. \& Sánchez, H.J., 2001. Analysis of arsenic pollution in groundwater aquifers by X-ray fluorescence. Applied radiation and isotopes : including data, instrumentation and methods for use in agriculture, industry and medicine, 54(5), pp.737-40. Available at: http://www.ncbi.nlm.nih.gov/pubmed/11258520.

Selck, D.A. et al., 2013. Increased Robustness of Single-Molecule Counting with Micro fl uidics, Digital Isothermal Ampli fi cation, and a Mobile Phone versus Real-Time Kinetic Measurements. Analytical chemistry, 85, pp.11129-11136.

Sharma, R.D., Joshi, S. \& Amlathe, S., 2011. MATLAB assisted disposable sensors for quantitative determination of arsenic. Analytical Methods, 3(2), p.452. Available at: http://xlink.rsc.org/?DOI=c0ay00584c [Accessed November 27, 2014].

Shen, L., Hagen, J. a \& Papautsky, I., 2012. Point-of-care colorimetric detection with a smartphone. Lab on a chip, 12(21), pp.4240-3. Available at: http://www.ncbi.nlm.nih.gov/pubmed/22996728 [Accessed March 25, 2014].

Shrivastava, A. \& Gupta, V.B., 2011. Methods for the determination of limit of detection and limit of quantitation of the analytical methods. Chronicles of Young Scientists, 2(1), pp.21-25.

Sicard, C. et al., 2015. Tools for water quality monitoring and mapping using paper-based sensors and cell phones Cl. Water Researh, 70, pp.360-369.

Siegfried, K. et al., 2012. Field testing of arsenic in groundwater samples of Bangladesh using a test kit based on lyophilized bioreporter bacteria. Environmental science \& technology, 46(6), pp.3281-7. Available at: http://www.ncbi.nlm.nih.gov/pubmed/22339623.

Skoog, D.A., Holler, F.J. \& Crouch, S.R., 2006. Principles of Instrumental Analysis 6th ed., Cengage Learning. 
Smedley, P.L. \& Kinniburgh, D.G., 2002. A review of the source, behaviour and distribution of arsenic in natural waters. Applied Geochemistry, 17, pp.517-568.

Smith, A.H., Lingas, E.O. \& Rahman, M., 2000. Contamination of drinking-water by arsenic in Bangladesh: a public health emergency. Bulletin of the World Health Organization, 78(9), pp.10931103. Available at:

http://www.pubmedcentral.nih.gov/articlerender.fcgi?artid=2560840\&tool=pmcentrez\&rendertype= abstract.

Sohel, N. et al., 2009. Arsenic in drinking water and adult mortality: a population-based cohort study in rural Bangladesh. Epidemiology (Cambridge, Mass.), 20(6), pp.824-30. Available at: http://www.ncbi.nlm.nih.gov/pubmed/19797964 [Accessed April 22, 2014].

Stedman, D.H. et al., 1983. Chemiluminescent Detection of Arsine Oxidation. Analytical chemistry, 55, pp.1809-1810.

Steinmaus, C.M. et al., 2006. Evaluation of two new arsenic field test kits capable of detecting arsenic water concentrations close to 10 microg/L. Environmental science \& technology, 40(10), pp.3362-6. Available at: http://www.ncbi.nlm.nih.gov/pubmed/16749706.

Stocker, J. et al., 2003. Development of a set of simple bacterial biosensors for quantitative and rapid measurements of arsenite and arsenate in potable water. Environmental science \& technology, 37(20), pp.4743-50. Available at: http://www.ncbi.nlm.nih.gov/pubmed/14594387.

Stoytcheva, M., Sharkova, V. \& Panayotova, M., 1998. Electrochemical approach in studying the inhibition of acetylcholinesterase by arsenate(III): analytical characterisation and application for arsenic determination. Analytica Chimica Acta, 364(1-3), pp.195-201. Available at: http://linkinghub.elsevier.com/retrieve/pii/S0003267098001342.

Strehle, K.R. et al., 2007. A reproducible surface-enhanced raman spectroscopy approach. Online SERS measurements in a segmented microfluidic system. Analytical chemistry, 79(4), pp.1542-7. Available at: http://www.ncbi.nlm.nih.gov/pubmed/17297953.

Taokaenchan, N. et al., 2014. Specific speciation of As ( III ) and As ( V ) in aqueous solution by a split microfluidic chemiluminescence system. , 31(1), pp.27-31.

Theriault, G. a., Bodensteiner, S. \& Lieberman, S.H., 1998. A real-time fiber-optic LIBS probe for the in situ delineation of metals in soils. Field Analytical Chemistry \& Technology, 2(2), pp.117-125. Available at: http://doi.wiley.com/10.1002/(SICI)1520-6521(1998)2:2<117::AIDFACT8>3.0.CO;2-T.

Theytaz, J. et al., 2009. Biochip with E. coli bacteria for detection of arsenic in drinking water. Procedia Chemistry, 1(1), pp.1003-1006. Available at: http://linkinghub.elsevier.com/retrieve/pii/S1876619609002514 [Accessed February 1, 2014].

Thirunavukkarasu, O.S., Viraraghavan, T. \& Subramanian, K.S., 2003. Arsenic removal from drinking water using iron oxide-coated sand. Water, Air, \& Soil Pollution, 142, pp.95-111. 
Truffer, F. et al., 2014. Compact portable biosensor for arsenic detection in aqueous samples with Escherichia coli bioreporter cells Compact portable biosensor for arsenic detection in aqueous samples with Escherichia coli bioreporter cells. , 015120, pp.10-14.

Tseng, C.-H., 2002. An Overview on Peripheral Vascular Disease in Blackfoot Disease-Hyperendemic Villages in Taiwan. Angiology, 53(5), pp.529-537. Available at: http://ang.sagepub.com/cgi/doi/10.1177/000331970205300505 [Accessed April 22, 2014].

Tsuda, T. et al., 1995. Ingested arsenic and internal cancer: a historical cohort study followed for 33 years. American journal of epidemiology, 141(3), pp.198-209. Available at: http://www.ncbi.nlm.nih.gov/pubmed/7840093.

United States Environmental Protection Agency Office of Water, 1999. Analytical Methods Support Document For Arsenic In Drinking Water,

Vernekar, V.N. \& Latour, R.A., 2005. Adsorption Thermodynamics Of A Mid-Chain Peptide Residue On Functionalized SAM Surfaces Using SPR. Materils Research Innovations Online, 9(2), pp.337-353.

Villanueva, C. \& Kogevinas, M., 1999. Comments on "Drinking water arsenic in Utah: a cohort mortality study". Environmental health perspectives, 107(11), pp.359-365. Available at: http://www.pubmedcentral.nih.gov/articlerender.fcgi?artid=1566713\&tool=pmcentrez\&rendertype= abstract.

Wagli, P. et al., 2013. Microfluidic droplet-based liquid-liquid extraction and on-chip IR spectrscopy detection of cocaine in human saliva. Analytical chemistry, 85, pp.7558-7565.

Wahed, M. a et al., 2006. A modified routine analysis of arsenic content in drinking-water in Bangladesh by hydride generation-atomic absorption spectrophotometry. Journal of health, population, and nutrition, 24(1), pp.36-41. Available at: http://www.ncbi.nlm.nih.gov/pubmed/16796148.

Walter, A. et al., 2011. Towards a fast, high specific and reliable discrimination of bacteria on strain level by means of SERS in a microfluidic device. Lab on a chip, 11(6), pp.1013-21. Available at: http://www.ncbi.nlm.nih.gov/pubmed/21283864 [Accessed May 6, 2014].

Wang, Z. et al., 2007. Surface plasmon resonance imaging for affinity analysis of aptamer-protein interactions with PDMS microfluidic chips. Analytical and bioanalytical chemistry, 389(3), pp.81925. Available at: http://www.ncbi.nlm.nih.gov/pubmed/17673982 [Accessed May 4, 2014].

Wei, Y. \& Latour, R. a, 2008. Determination of the adsorption free energy for peptide-surface interactions by SPR spectroscopy. Langmuir : the ACS journal of surfaces and colloids, 24(13), pp.6721-9. Available at: http://www.pubmedcentral.nih.gov/articlerender.fcgi?artid=2638991\&tool=pmcentrez\&rendertype= abstract.

Weiner, M., 2000. Chelation: Therapy or “Therapy"? National Capital Poison Center, 189(5). Available at: http://www.poison.org/current/chelationtherapy.htm. 
Whitesides, G.M., 2006. The origins and the future of microfluidics. Nature, 442(7101), pp.368-73. Available at: http://www.ncbi.nlm.nih.gov/pubmed/16871203 [Accessed September 19, 2013].

WHO, 2011. Arsenic in Drinking-water: Background document for development of WHO Guidelines for Drinking Water Quality,

WHO, 2001. Water-related diseases: Arsenicosis,

World Bank Water and Sanitation Program, 2005. Towards a more effective operational response: arsenic contamination of ground water in South and East Asian countries, Available at: http://siteresources.worldbank.org/SOUTHASIAEXT/Images/223545-1202240835749/46430381203396959973/4683213-1258489308659/WLD33757.jpg.

Wu, M.M. et al., 1989. Dose-response relation between arsenic concentration in well water and mortality from cancers and vascular diseases. American journal of epidemiology, 130(6), pp.1123-32. Available at: http://www.ncbi.nlm.nih.gov/pubmed/2589305.

Wu, Y., Zhan, S., et al., 2012. Cationic polymers and aptamers mediated aggregation of gold nanoparticles for the colorimetric detection of arsenic(III) in aqueous solution. Chemical communications (Cambridge, England), 48(37), pp.4459-61. Available at: http://www.ncbi.nlm.nih.gov/pubmed/22453203 [Accessed February 10, 2014].

$\mathrm{Wu}, \mathrm{Y}$., Liu, L., et al., 2012. Ultrasensitive aptamer biosensor for arsenic(III) detection in aqueous solution based on surfactant-induced aggregation of gold nanoparticles. The Analyst, 137(18), pp.4171-8. Available at: http://www.ncbi.nlm.nih.gov/pubmed/22842645 [Accessed April 9, 2014].

Xia, N. et al., 2012. Simple, rapid and label-free colorimetric assay for arsenic based on unmodified gold nanoparticles and a phytochelatin-like peptide. Analytical Methods, 4(12), p.3937. Available at: http://xlink.rsc.org/?DOI=c2ay25803j [Accessed November 11, 2013].

Xiao, L., Wildgoose, G.G. \& Compton, R.G., 2008. Sensitive electrochemical detection of arsenic (III) using gold nanoparticle modified carbon nanotubes via anodic stripping voltammetry. Analytica chimica acta, 620(1-2), pp.44-9. Available at: http://www.ncbi.nlm.nih.gov/pubmed/18558122 [Accessed March 25, 2014].

Xu, B.-B. et al., 2011. Localized flexible integration of high-efficiency surface enhanced Raman scattering (SERS) monitors into microfluidic channels. Lab on a chip, 11(19), pp.3347-51. Available at: http://www.ncbi.nlm.nih.gov/pubmed/21863148 [Accessed May 6, 2014].

Yang, C.-Y. et al., 2005. Detection of picomolar levels of interleukin-8 in human saliva by SPR. Lab on $a$ chip, 5(10), pp.1017-23. Available at: http://www.ncbi.nlm.nih.gov/pubmed/16175255 [Accessed May 4, 2014].

Yang, C.-Y. et al., 2008. Is colon cancer mortality related to arsenic exposure? Journal of toxicology and environmental health. Part A, 71(8), pp.533-8. Available at: http://www.ncbi.nlm.nih.gov/pubmed/18338288 [Accessed April 22, 2014]. 
Yang, C.-Y. et al., 2004. Reduction in kidney cancer mortality following installation of a tap water supply system in an arsenic-endemic area of Taiwan. Archives of environmental health, 59(9), pp.484-8. Available at: http://www.ncbi.nlm.nih.gov/pubmed/16381491 [Accessed April 22, 2014].

Yavuz, C.T. et al., 2010. Pollution magnet: nano-magnetite for arsenic removal from drinking water. Environmental geochemistry and health, 32(4), pp.327-34. Available at: http://www.ncbi.nlm.nih.gov/pubmed/20440636 [Accessed October 3, 2013].

Yean, S. \& Cong, L., 2005. Effect of magnetite particle size on adsorption and desorption of arsenite and arsenate., (Iii), pp.3255-3264.

Zhu, H. et al., 2011. Optofluidic fluorescent imaging cytometry on a cell phone. Analytical chemistry, 83(17), pp.6641-7. Available at: http://www.pubmedcentral.nih.gov/articlerender.fcgi?artid=3263930\&tool=pmcentrez\&rendertype= abstract. 